\title{
Pesticide Inputs to the Sacramento-San Joaquin Delta, 2015-16: Results from the Delta Regional Monitoring Program
} 24.

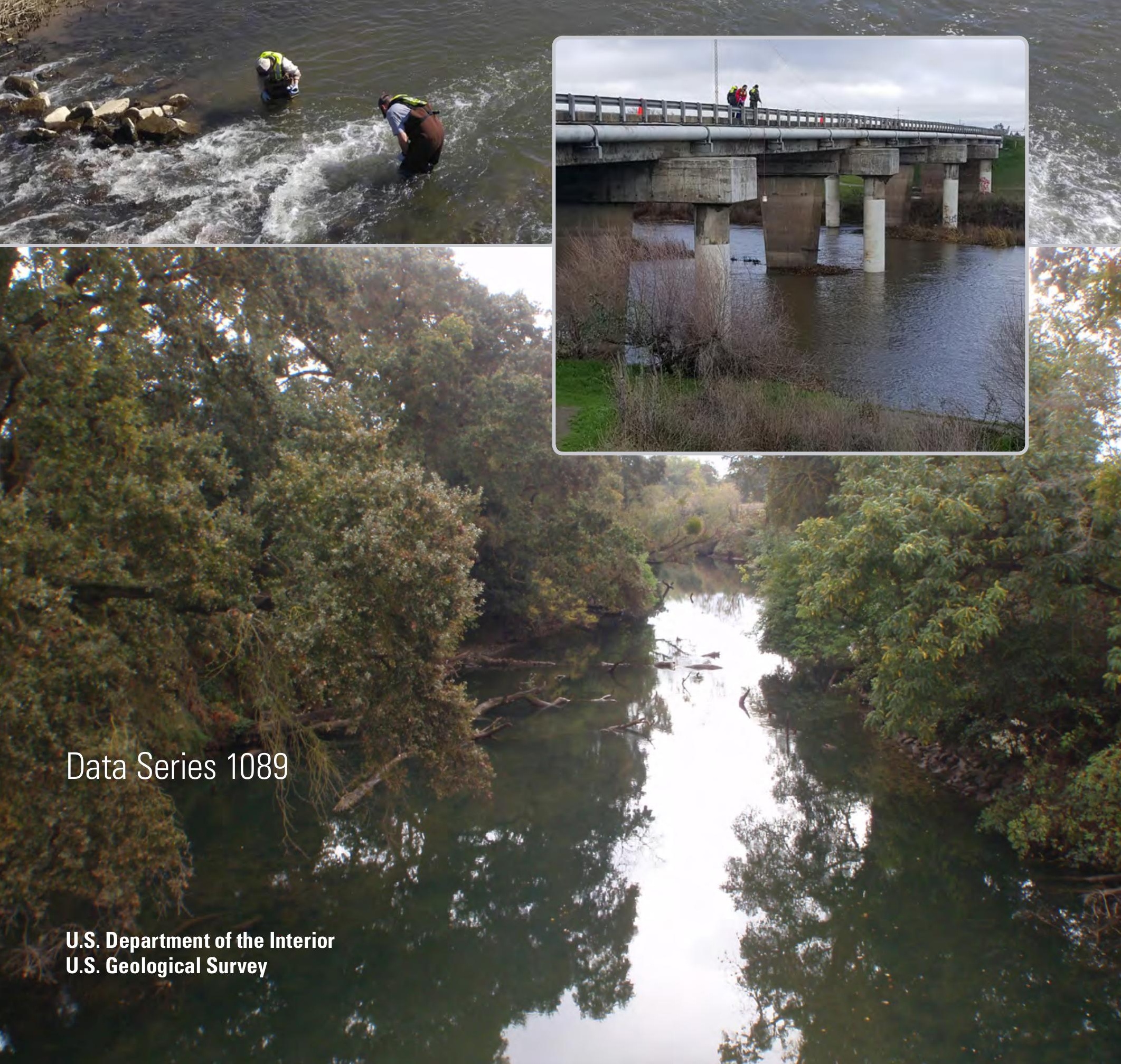




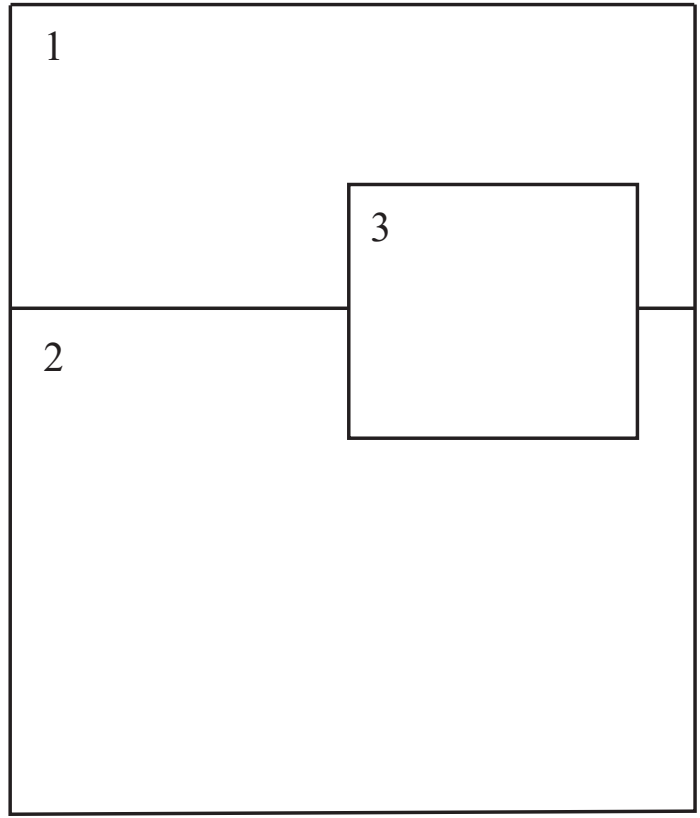

Cover. 1. U.S. Geological Survey personnel preparing to collect water samples from Ulatis Creek at Browns Road near Elmira, California. 2. Mokelumne River at New Hope Road at Thornton, California, looking upstream.

3. View of bridge over the San Joaquin River near Vernalis, California. 


\section{Pesticide Inputs to the Sacramento- San Joaquin Delta, 2015-16: Results from the Delta Regional Monitoring Program}

By Matthew De Parsia, James L. Orlando, Megan M. McWayne, and

Michelle L. Hladik

Prepared in cooperation with the Delta Regional Monitoring Program

Data Series 1089 


\title{
U.S. Department of the Interior \\ RYAN K. ZINKE, Secretary
}

\author{
U.S. Geological Survey \\ James F. Reilly II, Director
}

U.S. Geological Survey, Reston, Virginia: 2018

For more information on the USGS - the Federal source for science about the Earth, its natural and living resources, natural hazards, and the environment—visit http://www.usgs.gov or call 1-888-ASK-USGS (1-888-275-8747).

For an overview of USGS information products, including maps, imagery, and publications, visit http://store.usgs.gov.

Any use of trade, firm, or product names is for descriptive purposes only and does not imply endorsement by the U.S. Government.

Although this information product, for the most part, is in the public domain, it also may contain copyrighted materials as noted in the text. Permission to reproduce copyrighted items must be secured from the copyright owner.

Suggested citation:

De Parsia, M., Orlando, J.L., McWayne, M.M., and Hladik, M.L., 2018, Pesticide inputs to the Sacramento- San Joaquin Delta, 2015-16: Results from the Delta Regional Monitoring Program: U.S. Geological Survey Data Series 1089, 49 p., https://doi.org/10.3133/ds1089

ISSN 2327-638X (online) 


\section{Contents}

Abstract

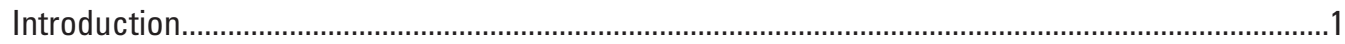

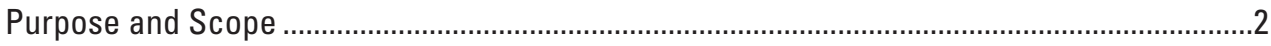

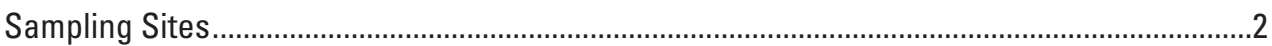

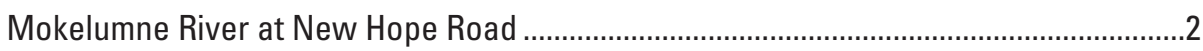

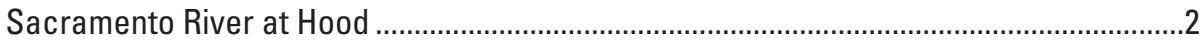

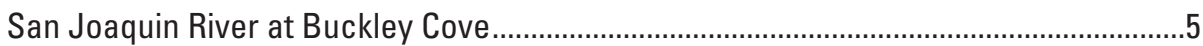

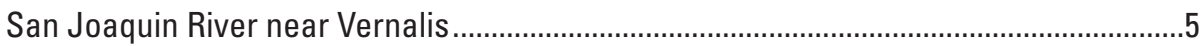

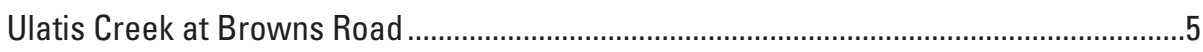

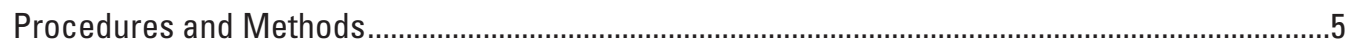

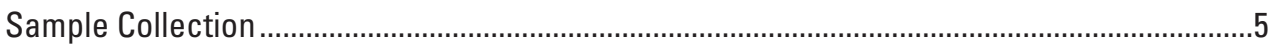

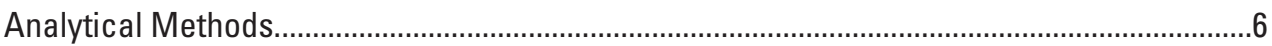

Organic Chemistry Research Laboratory, Sacramento, California ....................................6

Extraction of Pesticides from Surface Water ..........................................................

Extraction of Pesticides from Suspended Sediment ...............................................6

Instrument Methods .................................................................................................

National Water Quality Laboratory, Denver, Colorado ………………...............................

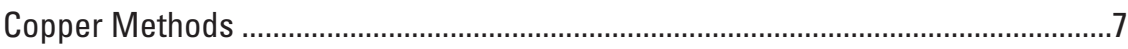

Inorganic Carbon, Organic Carbon, and Nitrogen Methods ....................................

Method Detection Limits and Reporting Levels...................................................................

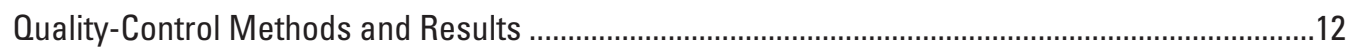

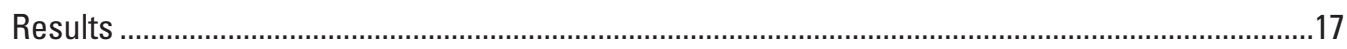

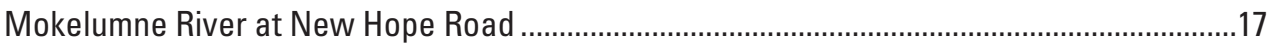

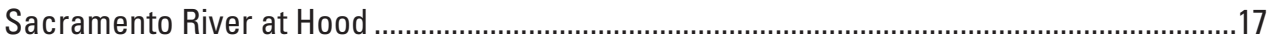

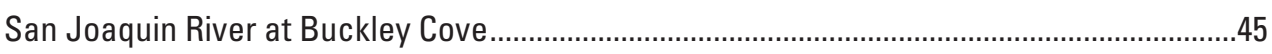

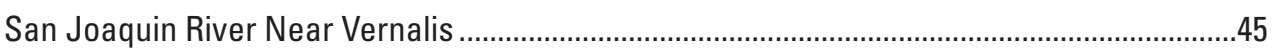

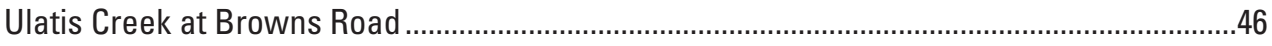

Summary

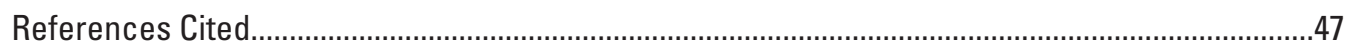

\section{Figures}

1. Map showing sampling sites and the legal boundary of the SacramentoSan Joaquin Delta, 2015-16.........................................................................................

2. Map showing land-use types and watershed boundaries for sites sampled from July 2015 to June 2016 in the Sacramento-San Joaquin Delta, California................4

3. Graph showing numbers of pesticides detected per month in surface-water samples from July 2015 to June 2016 in the Sacramento-San Joaquin Delta, California. 


\section{Tables}

1. Surface-water sampling sites located in the Sacramento-San Joaquin Delta, 2015-16..

2. Method detection limits for dissolved pesticides in surface water and on suspended sediments measured by the U.S. Geological Survey Organic Chemistry Research Laboratory.....

3. Reporting levels for dissolved and suspended consituents measured by the U.S. Geological Survey National Water Quality Laboratory.

4. Pesticide concentrations with measured detections by gas chromatography with mass spectrometry (GC/MS) in environmental and field replicate water samples collected in the Sacramento-San Joaquin Delta, 2015-16

5. Pesticide concentrations with measured detections by liquid chromatography tandem mass spectrometry (LC/MS/MS) in environmental and field replicate water samples collected in the Sacramento-San Joaquin Delta, 2015-16..

6. Minimum, maximum, and median recovery of compounds in pesticide matrix-spiked water samples with standard deviation.

7. Pesticide concentrations with measured detections in environmental water samples collected in the Sacramento-San Joaquin Delta, 2015-16.

8. Pesticide concentrations with measured detections in suspended sediments filtered from environmental water samples collected in the Sacramento-San Joaquin Delta, 2015-16.

9. Whole water pesticide concentrations for all compounds with detections in water and suspended sediment filtered from environmental water samples collected in the Sacramento-San Joaquin Delta, 2015-16.

10. Concentrations of dissolved and suspended constituents measured in environmental water samples collected in the SacramentoSan Joaquin Delta, 2015-16.

11. Water quality field parameters measured in surface-water samples collected in the Sacramento-San Joaquin Delta, California, 2015-16. 


\section{Conversion Factors}

International System of Units to U.S. customary units

\begin{tabular}{lll}
\hline \multicolumn{1}{c}{ Multiply } & \multicolumn{1}{c}{ Length } & \multicolumn{1}{c}{ To obtain } \\
\hline micrometer $(\mu \mathrm{m})$ & 0.00003937 & inch (in.) \\
millimeter $(\mathrm{mm})$ & 0.03937 & inch (in.) \\
meter $(\mathrm{m})$ & 3.281 & foot (ft) \\
\hline \multicolumn{1}{c}{ Area } & \\
\hline square kilometer $(\mathrm{km} 2)$ & 0.3861 & square mile (mi2) \\
\hline \multicolumn{1}{c}{ Volume } & \\
\hline milliliter $(\mathrm{mL})$ & 0.033814 & ounce, fluid (fl. oz) \\
liter $(\mathrm{L})$ & 33.81402 & ounce, fluid (fl. oz) \\
\hline \multicolumn{1}{c}{ Flow rate } & \\
\hline milliliter per minute $(\mathrm{mL} / \mathrm{min})$ & 0.033814 & ounce, fluid per minute (fl. oz/min) \\
\hline & \multicolumn{1}{c}{ Mass } & \\
\hline milligram $(\mathrm{mg})$ & 0.000035 & ounce, (oz) \\
kilogram $(\mathrm{kg})$ & 2.20462 & pound, (lb) \\
\hline
\end{tabular}

Temperature in degrees Celsius $\left({ }^{\circ} \mathrm{C}\right)$ may be converted to degrees Fahrenheit $\left({ }^{\circ} \mathrm{F}\right)$ as follows:

${ }^{\circ} \mathrm{F}=\left(1.8 \times{ }^{\circ} \mathrm{C}\right)+32$.

\section{Datum}

North American Datum of 1983 (NAD 83).

\section{Supplemental Information}

Specific conductance is given in microsiemens per centimeter at 25 degrees Celsius $(\mu \mathrm{S} / \mathrm{cm}$ at $\left.25^{\circ} \mathrm{C}\right)$.

Concentrations of chemical constituents in water are given in either milligrams per liter (mg/L), micrograms per liter $(\mu \mathrm{g} / \mathrm{L})$, or nanograms per liter $(\mathrm{ng} / \mathrm{L})$. One milligram per liter is equivalent to 1 part per million (ppm); one microgram per liter is equivalent to 1 part per billion (ppb); one nanogram per liter is equivalent to 1 part per trillion (ppt). Concentrations of pesticide compounds in suspended-sediments filtered from 1-liter water samples are provided in $\mathrm{ng} / \mathrm{L}$ to facilitate the approximation of a whole-water pesticide concentration by summing the dissolved and suspended-sediment concentrations of pesticide compounds. 


\section{Abbreviations}

\begin{tabular}{|c|c|}
\hline AHP & Aquatic Health Program \\
\hline ASC & Aquatic Science Center \\
\hline CDPR & California Department of Pesticide Regulation \\
\hline Delta RMP & Delta Regional Monitoring Program \\
\hline DOC & dissolved organic carbon \\
\hline EtOAc & ethyl acetate \\
\hline EPA & U.S. Environmental Protection Agency \\
\hline GC/MS & gas chromatography mass spectrometry \\
\hline LC/MS/MS & liquid chromatography tandem mass spectrometry \\
\hline LT-MDL & long-term method detection level \\
\hline MDL & method detection limit \\
\hline MRL & minimum reporting level \\
\hline NWIS & National Water Information System \\
\hline NWQL & National Water Quality Laboratory \\
\hline OCRL & Organic Chemistry Research Laboratory \\
\hline PIC & particulate inorganic carbon \\
\hline \pm & plus or minus \\
\hline POC & particulate organic carbon \\
\hline POD & pelagic organism decline \\
\hline QAPP & quality assurance program plan \\
\hline RSD & relative standard deviation \\
\hline SPE & solid phase extraction \\
\hline SWRCB & State Water Resources Control Board \\
\hline TPC & total particulate carbon \\
\hline TPN & total particulate nitrogen \\
\hline UCD & University of California, Davis \\
\hline USGS & U.S. Geological Survey \\
\hline
\end{tabular}




\title{
Pesticide Inputs to the Sacramento-San Joaquin Delta, 2015-16: Results from the Delta Regional Monitoring Program
}

\author{
By Matthew De Parsia, James L. Orlando, Megan M. McWayne, and Michelle L. Hladik
}

\section{Abstract}

Emergent hypotheses about causes of the pelagic organism decline in the Sacramento-San Joaquin Delta (Delta) indicate that a more complete understanding of the quality of water entering the Delta is needed. Less than half of all pesticides used in the Delta watershed are measured in samples collected for routine monitoring, and with new pesticides continually being registered for use, the concentrations of unmonitored pesticides in the Delta ecosystem are unknown. In response, a multi-year, cooperative effort to improve monitoring of mercury, nutrients, pathogens, and pesticides was begun by the Delta Regional Monitoring Program (RMP). In July 2015, the U.S. Geological Survey in cooperation with the Delta RMP began measuring concentrations of 154 pesticide compounds in monthly samples of surface water and suspended sediment collected at five major inputs to the Sacramento-San Joaquin Delta from July 2015 to June 2016. In addition to pesticide concentration measurements, field water-quality indicators (water temperature, specific conductance, dissolved oxygen, $\mathrm{pH}$, and turbidity) were measured at each site and samples were collected for the analysis of dissolved organic carbon, dissolved copper, particulate organic carbon, particulate inorganic carbon, total particulate carbon, and total particulate nitrogen. Pesticide concentrations in particulates were measured in collected suspended-sediment samples by gas chromatography with mass spectrometry, whereas concentrations measured in surface-water samples utilized a combination of gas chromatography with mass spectrometry and liquid chromatography with tandem mass spectrometry. Samples were collected from two sites in the San Joaquin River watershed and at one site for each of the Mokelumne River, Sacramento River, and Ulatis Creek watersheds.

All water samples contained mixtures of 2-25 pesticides. Pesticides were detected in 100 percent of surfacewater samples. A total of 54 pesticide compounds were detected in water samples during the study period (19 fungicides, 18 herbicides, 9 insecticides, 7 breakdown products, and 1 synergist). The most frequently detected pesticide compounds were the herbicides hexazinone (95 percent) and diuron ( 73 percent) and the fungicides boscalid (93 percent) and azoxystrobin (75 percent). Pesticide concentrations ranged from below the method detection limits to 2,630 nanograms per liter for the herbicide metolachlor.

A total of 11 pesticide compounds were detected in the suspended sediments filtered from water samples (6 herbicides, 3 insecticides, 1 fungicide, and 1 breakdown product). The most frequently detected compounds were the insecticides permethrin ( 7 percent) and bifenthrin (5 percent) and the herbicide pendimethalin (5 percent). Pesticide concentrations in the suspended-sediment ranged from below the method detection limit to 265 nanograms per liter for the herbicide pendimethalin.

\section{Introduction}

The Sacramento-San Joaquin Delta (Delta) is an area of great importance to humans as both a source and a transport mechanism of freshwater. The Delta is also an area of critical habitat for numerous threatened and endangered species of concern, including Chinook salmon (Oncorhynchus tshawytscha) and the Delta smelt (Hypomesus transpacificus; Moyle, 2002; Brown and Moyle, 2005; Sommer and Mejia, 2013). In recent years, multiple pelagic species in the Delta have been in sharp decline (Feyrer and others, 2007; Sommer and others, 2007). This phenomenon, termed the pelagic organism decline (POD), is thought to have four causes: (1) previous abundance of pelagic species; (2) changes in habitat, including changes in water quantity and water quality; (3) top-down effects, including predation and losses caused by entrainment in water export pumps; and (4) bottom-up changes in food availability for pelagic species (Baxter and others, 2010). These factors point to the need for a complete and timely understanding of the quality of water entering the Delta.

Studies have indicated that contaminants, including pesticides, may play a role in the POD (Sommer and others, 2007; Werner and others, 2010). In 2014, reported pesticide 
use in the Delta watershed was over 50 million pounds of active ingredient (California Department of Pesticide Regulation, 2016). Because year-to-year pesticide use is continually changing, it presents a challenge for resource managers and policy makers trying to understand the fate and effects of these contaminants. Additionally, accurate estimates of pesticide use are difficult to make because only licensed pesticide applicators are required to report pesticide use to the California Department of Pesticide Regulation (CDPR). State regulators do not track household pesticide use and pesticides used in seed coatings.

Previous studies have shown that pesticides associated with agricultural and urban runoff are present in the Delta throughout the year and that the types and concentrations of these pesticides vary based on their use in the upstream watersheds (Dileanis and others, 2002; Kratzer and others, 2002; Zamora and others, 2003; Orlando and Kuivila, 2005; Weston and Lydy, 2010; Zhang and others, 2012; Orlando and others, 2013; Orlando and others, 2014). Finally, less than half of all pesticides applied in the Delta watershed are analyzed for during routine monitoring studies, and new pesticides are continually being registered for use (Kuivila and Hladik, 2008). The concentrations and potential effects of these unmonitored pesticides in the Delta ecosystem are unknown.

This study was conducted in cooperation with the San Francisco Estuary Institute Aquatic Science Center as part of the Delta Regional Monitoring Program (Delta RMP), a multi-year, cooperative effort to better track beneficial-use protections and restoration efforts in the Delta through the monitoring of mercury, nutrients, pathogens, and pesticides (Aquatic Science Center, 2017). The Delta RMP was created by the Central Valley Regional Water Quality Control Board to better coordinate water-quality monitoring in response to the early 2000s decline of pelagic fish species in the Delta. The Aquatic Science Center (ASC), a joint powers authority created by the State Water Resources Control Board (SWRCB) and the Bay Area Clean Water Agencies, is responsible for implementing activities necessary to achieve the goals of the Delta RMP, as well as preparing and publishing results collected for the program (Aquatic Science Center, 2017). Monthly pesticide monitoring for the Delta RMP began in July 2015 and concluded in June 2017; this report contains results for July 2015 through June 2016. Although monthly pesticide monitoring concluded in June 2017, the Delta RMP is an ongoing monitoring program with no set end date for data collection or data dissemination.

Pesticide and toxicity samples were collected concurrently to determine whether pesticides could contribute to observed toxicity in the Delta. The role of the USGS in the Delta RMP is to collect pesticide, toxicity, and ancillary waterquality (dissolved organic carbon, DOC; dissolved copper; particulate organic carbon, POC; particulate inorganic carbon, PIC; total particulate carbon, TPC; and total particulate nitrogen, TPN) samples; perform the pesticide and ancillary water-quality analyses; and to generate pesticide-detection reports. Surface-water samples also were collected by the
USGS for toxicity analysis by the University of California, Davis (UCD); ASC plans to publish these toxicity results in a future monitoring report.

\section{Purpose and Scope}

This report describes field and laboratory methods used and reports the measured concentrations of pesticides and pesticide degradates found in surface water and in particulates filtered from surface-water samples at five sites that provide surface water input to the Delta. Concentrations of organic carbon, inorganic carbon, nitrogen, and copper also were measured and reported. Constituents with detections above the aquatic-life benchmark for chronic toxicity to invertebrates (U.S. Environmental Protection Agency, 2017) are reported where applicable. The data-collection period of July 2015June 2016 represents data collected for the first year of the Delta RMP.

\section{Sampling Sites}

Five sites were selected to be sampled monthly at inputs to the Delta (fig. 1; table 1). These sites were chosen by the Delta RMP Technical Advisory Committee to capture water at representative inflows to the Delta, to cover an extensive area, and to complement existing monitoring datasets (Aquatic Science Center, 2017). Land-use types and watershed boundaries for the five sampling sites are shown in figure 2. Land use has been grouped into six broad categories: urban, bare ground, forest, shrub and grasslands, agricultural, or wetlands (U.S. Geological Survey, 2014). The watershed boundary for the San Joaquin River at Buckley Cove also includes the San Joaquin River at Vernalis watershed boundary.

\section{Mokelumne River at New Hope Road}

The Mokelumne River originates in the western slope of the Sierra Nevada and drains into the San Joaquin River. Samples were collected from a bridge over the Mokelumne River. The site is tidally influenced; therefore, samples were only collected during outgoing tides. The contributing watershed area is approximately 700 square miles $\left(\mathrm{mi}^{2}\right)$, and land use is 8 percent agricultural and 2 percent urban (U.S. Geological Survey, 2014). The primary crops grown in the watershed by area are grapes, walnuts, and alfalfa (National Agricultural Statistics Service, 2016).

\section{Sacramento River at Hood}

The Sacramento River flows south from its headwaters in northern California and drains into the Delta. Samples at this site were collected 30 feet (ft) from the left bank on the Sacramento River from a monitoring platform maintained by the SWRCB. This site has minimal tidal influence, and although 


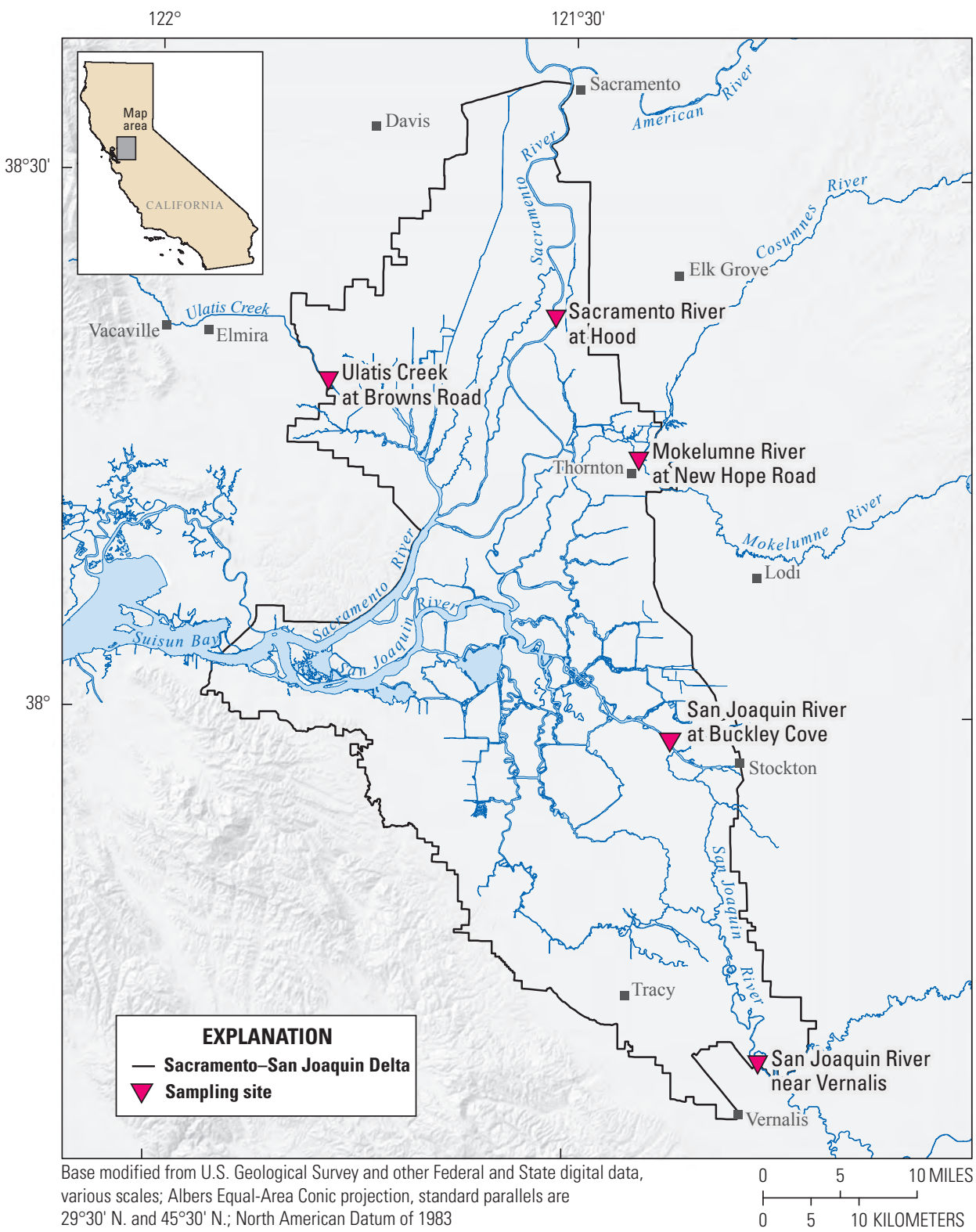

Figure 1. Sampling sites and the legal boundary of the Sacramento-San Joaquin Delta, 2015-16.

Table 1. Surface-water sampling sites located in the Sacramento-San Joaquin Delta, 2015-16.

[Calif., California; dms, degree minute second; ID, identification; USGS, U.S. Geological Survey; ${ }^{\circ}$, degree; ', minute; ", second; *, collected from bridge at mid-channel during high-flow events]

\begin{tabular}{llllll}
\hline $\begin{array}{c}\text { USGS station } \\
\text { number }\end{array}$ & \multicolumn{1}{c}{ USGS station name } & \multicolumn{1}{c}{ Field ID } & $\begin{array}{c}\text { Latitude1 } \\
\text { (dms) }\end{array}$ & $\begin{array}{c}\text { Longtitude1 } \\
\text { (dms) }\end{array}$ & $\begin{array}{c}\text { Sample collection } \\
\text { point }\end{array}$ \\
\hline 381411121250901 & $\begin{array}{c}\text { Mokelumne River at New Hope } \\
\text { Road at Thornton, Calif. }\end{array}$ & $\begin{array}{c}\text { Mokelumne River at New } \\
\text { Hope Road }\end{array}$ & $38^{\circ} 14^{\prime} 11^{\prime \prime}$ & $121^{\circ} 25^{\prime} 09^{\prime \prime}$ & Bridge, mid-channel \\
382205121311300 & Sacramento River at Hood, Calif. & Sacramento River at Hood & $38^{\circ} 22^{\prime} 05^{\prime \prime}$ & $121^{\circ} 31^{\prime} 13^{\prime \prime}$ & Catwalk, mid-channel \\
375831121223701 & $\begin{array}{c}\text { San Joaquin River at Buckley } \\
\text { Cove near Stockton, Calif. } \\
11303500\end{array}$ & $\begin{array}{c}\text { San Joaquin River at } \\
\text { Buckley Cove }\end{array}$ & $37^{\circ} 58^{\prime} 31^{\prime \prime}$ & $121^{\circ} 22^{\prime} 37^{\prime \prime}$ & Wading, bank \\
11455261 & $\begin{array}{c}\text { San Joaquin River near Vernalis, } \\
\text { Calif. } \\
\text { Ulatis Creek at Browns Road } \\
\text { near Elmira, Calif. }\end{array}$ & $\begin{array}{c}\text { Sanuin River near } \\
\text { Vernalis } \\
\text { Ulatis Creek at Browns } \\
\text { Road }\end{array}$ & $37^{\circ} 40^{\prime} 34^{\prime \prime}$ & $121^{\circ} 15^{\prime} 59^{\prime \prime}$ & Wading, mid-channel* \\
\hline
\end{tabular}

${ }^{1}$ All locations reference North American Datum of 1983. 


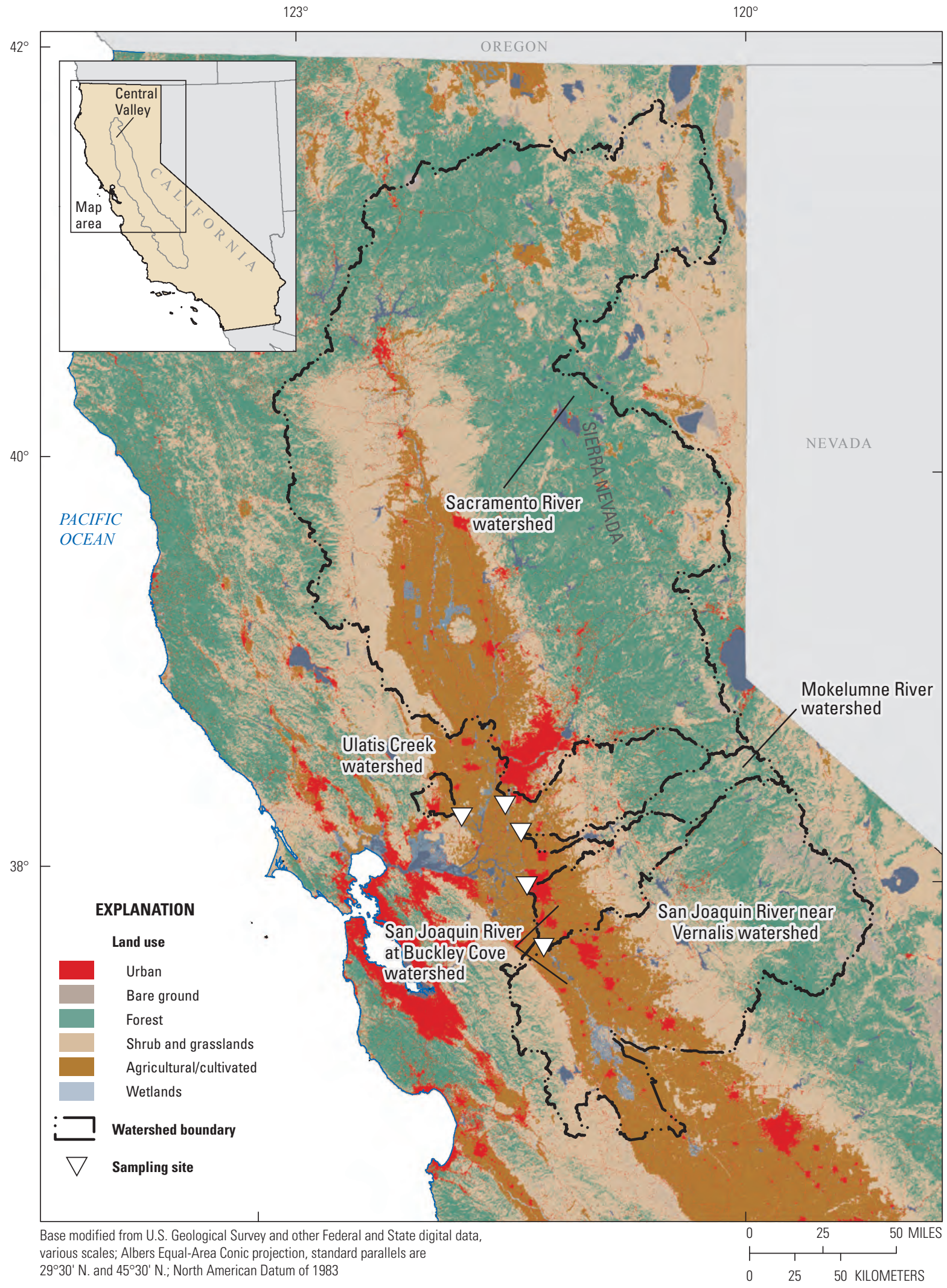

Figure 2. Land-use types and watershed boundaries for sites sampled from July 2015 to June 2016 in the Sacramento-San Joaquin Delta, California. 
flows do not regularly reverse directions, sample collection times were limited to outgoing tides to be sure to capture inflows to the Delta only. The watershed area upstream of the sample site encompasses over 23,900 $\mathrm{mi}^{2}$ of land, of which 11 percent is used for agricultural purposes and 4 percent for urban (U.S. Geological Survey, 2014). The primary crops grown in the watershed by area are rice, almonds, and walnuts (National Agricultural Statistics Service, 2016).

\section{San Joaquin River at Buckley Cove}

The San Joaquin River originates in the Sierra Nevada and flows northward through the Central Valley where it enters the Delta near Vernalis, Calif. (fig. 2). San Joaquin River at Buckley Cove is in an urban area, near the city of Stockton, Calif. (population 291,707; U.S. Census Bureau, 2016). Samples were collected from the right bank by wading into the river at this site. This site has a strong tidal influence, and samples were collected during outgoing tides. The watershed area includes the San Joaquin River at Vernalis watershed plus an additional 1,200 $\mathrm{mi}^{2}$ of land, including $120 \mathrm{mi}^{2}$ of urban land. The primary crops grown in the watershed by area are almonds, alfalfa, and walnuts (National Agricultural Statistics Service, 2016).

\section{San Joaquin River near Vernalis}

Flows on the San Joaquin River near Vernalis are not tidally influenced; they are highly influenced by dam releases and withdrawals for irrigation upstream of the sample collection point. Samples at this site were collected from a bridge over the San Joaquin River during high-flow conditions and collected by wading into the river during low-flow conditions. The watershed area upstream of the sample site encompasses over 7,300 $\mathrm{mi}^{2}$ of land; 21 percent of the land is used for agricultural purposes and 5 percent for urban (U.S. Geological Survey, 2014). The primary crops grown in the watershed by area are almonds, alfalfa, and grapes (National Agricultural Statistics Service, 2016).

\section{Ulatis Creek at Browns Road}

Ulatis Creek flows eastward from its source in the western margin of the Central Valley through the city of Vacaville, Calif. (population: 92,428; U.S. Census Bureau, 2016) to the valley floor where Ulatis Creek enters the Delta. Samples were collected from a bridge during high-flow conditions and by wading during low-flow conditions. The contributing watershed area is approximately $140 \mathrm{mi}^{2}$ with 18 percent of the area used for urban purposes and 44 percent for agriculture (U.S. Geological Survey, 2014). The primary crops grown in the watershed by area are alfalfa, almonds, and walnuts (National Agricultural Statistics Service, 2016).

\section{Procedures and Methods}

Surface-water samples for pesticide, water chemistry (organic carbon, inorganic carbon, particulate nitrogen, and copper), and toxicity analyses were collected concurrently at each site. Pesticide samples were analyzed by the USGS at the Organic Chemistry Research Laboratory (OCRL) in Sacramento, Calif. Dissolved and particulate organic carbon, particulate inorganic carbon, particulate nitrogen, and dissolved copper samples were analyzed by the USGS at the National Water Quality Laboratory (NWQL) in Denver, Colo. and toxicity samples were analyzed by the Aquatic Health Program (AHP) at UCD.

\section{Sample Collection}

Surface-water samples were collected monthly at all sites from July 2015 to June 2016. Ten sample sets were collected approximately mid-month, and two sample sets were collected following moderate-rainfall events on January 19, 2016, and March 7, 2016. Surface-water samples for pesticide, ancillary water-quality (copper, DOC, PIC, POC, TPC, and TPN), and toxicity analyses were collected concurrently at each site. All water samples were collected as grab samples in accordance with methods described in the USGS National Field Manual (U.S. Geological Survey, variously dated). The study design approved by the Delta RMP called for grab samples because of the large volume of water required for collecting toxicity and pesticide samples together, even in hydrologic conditions that might otherwise dictate integrated sampling techniques. Samples were collected between the high and low tides or ebb tide (for tidally influenced sites), by submerging narrowmouthed bottles at mid-channel to a depth of $1.5 \mathrm{ft}$. During low-flow conditions, samples were collected by wading into streams and submerging handheld bottles. In high-flow conditions or for sites with difficult bank access, samples were collected from bridges using weighted-bottle samplers.

Pesticide samples were collected in precleaned, baked glass-amber bottles and transported on ice to the USGS OCRL in Sacramento, Calif., for processing and analysis. Samples for analysis at the USGS NWQL were collected in Teflon ${ }^{\mathrm{TM}}$ bottles and transported on ice to the USGS California Water Science Center for processing. Prior to sampling, the Teflon ${ }^{\mathrm{TM}}$ bottles were cleaned with tap water and laboratory-grade detergent, rinsed with a 5 percent hydrochloric-acid solution, triple rinsed with ASTM Type-I deionized water, and stored in sealed plastic bags. The Teflon ${ }^{\mathrm{TM}}$ bottles were triple rinsed with native water prior to sample collection.

Toxicity samples were collected in precleaned, glassamber bottles provided by the AHP. Bottles were triple rinsed with native water on-site prior to sample collection. Ten bottles were collected at each site and transported on ice to the AHP for analysis.

Basic water-quality measurements (water temperature, specific conductance, dissolved oxygen, $\mathrm{pH}$, and turbidity) were taken at a depth of $1.5 \mathrm{ft}$ at mid-channel during each 
sample collection using a YSI 6920V2 multi-parameter meter. The meter was calibrated using appropriate procedures and standards prior to sample collection as described in the USGS National Field Manual (U.S. Geological Survey, variously dated).

\section{Analytical Methods}

Surface-water samples and suspended sediments filtered from surface water for pesticide analysis were analyzed by the USGS at the OCRL in Sacramento, Calif. Copper, DOC, PIC, POC, TPC, and TPN samples were analyzed by the USGS at the NWQL in Denver, Colo. Toxicity samples were analyzed by the AHP at UCD.

\section{Organic Chemistry Research Laboratory, Sacramento, California}

Samples for pesticide analysis were analyzed at the OCRL in Sacramento, Calif. One liter of water was extracted for analysis of 25 compounds by liquid chromatography tandem mass spectrometry (LC/MS/MS), and one liter of water was extracted for analysis of 129 compounds by gas chromatography mass spectrometry (GC/MS). All surfacewater samples for pesticide analysis were filtered through preweighed, baked 0.7-micrometer $(\mu \mathrm{m})$ glass-fiber filters (Grade GF/F, Whatman, Piscataway, N.J.) to remove suspended material. The filter paper from the GC/MS sample containing the suspended sediments was dried at room temperature overnight, protected from light, then stored in a freezer at -20 degrees Celsius $\left({ }^{\circ} \mathrm{C}\right)$ until extraction. Analysis of pesticides in suspended-material samples was done on resultant filter paper.

\section{Extraction of Pesticides from Surface Water}

The extraction procedure and instrumental analysis by LC/MS/MS have been previously described in Hladik and Calhoun (2012). To summarize the method described in Hladik and Calhoun (2012), filtered-water samples were spiked with the recovery surrogate standards, monuron (Chem Service, West Chester, Pennsylvania) and imidacloprid- $\mathrm{d}_{4}$ (Cambridge Isotope Laboratories, Andover, Massachusetts). Each sample was then passed through an Oasis Hydrophilic Lipophilic Balance (HLB) solid-phase extraction (SPE; 6 milliliters, $\mathrm{mL}$; 500 milligrams, mg; Waters, Milford, Mass.) cartridge that had been cleaned with one column volume of dichloromethane followed by one column volume of acetone and two column volumes of deionized water. During the SPE process, the water samples were pumped through the SPE cartridge at a flow rate of 10 milliliters per minute $(\mathrm{mL} / \mathrm{min})$ and the cartridge was then dried under nitrogen until the SPE sorbent was dry. The analytes were eluted with $10 \mathrm{~mL}$ of 50:50 DCM:acetone. The eluent was then evaporated to less than $0.5 \mathrm{~mL}$ using a gentle stream of dry nitrogen, solvent exchanged into acetonitrile, and further evaporated to $0.2 \mathrm{~mL}$. The internal standard $\left({ }^{13} \mathrm{C}_{3}\right.$-caffeine, Cambridge Isotope Laboratories, Andover,
Mass.) was then added. The sample extracts were stored in a freezer at $-20^{\circ} \mathrm{C}$ until analysis (up to 30 days).

The extraction procedure (Hladik and others, 2008, 2009) and instrumental analysis by GC/MS (Hladik and McWayne, 2012) have been previously described. Filtered-water samples were spiked with the recovery surrogate standards ${ }^{13} \mathrm{C}_{3}$-atrazine and $\mathrm{d}_{14}$-trifluralin (Cambridge Isotopes, Andover, Mass.). Each sample was then passed through an Oasis HLB SPE (6 mL, $500 \mathrm{mg}$, Waters, Milford, Mass.) cartridge that had been cleaned with two column volumes of ethyl acetate (EtOAc), followed by two column volumes of methanol, and two column volumes of deionized water. During this process, the water samples were pumped through the SPE cartridge at a flow rate of $10 \mathrm{~mL} / \mathrm{min}$ and the cartridge was dried under nitrogen until the SPE sorbent was dry. After extraction, sodium sulfate was added to the sample bottle to remove any residual water, then the bottle was rinsed three times with approximately $2 \mathrm{~mL}$ of DCM into a collection tube. The bottle rinse was concentrated to $1 \mathrm{~mL}$ under a gentle stream of nitrogen gas. The SPE cartridge was dried under nitrogen until the SPE sorbent was dry, then analytes were eluted with $12 \mathrm{~mL}$ of EtOAc into the concentrator tube containing the bottle rinse. The combined bottle rinse and eluent mixture was evaporated to less than $0.2 \mathrm{~mL}$ using a gentle stream of dry nitrogen. The internal standard, a mixture of deuterated compounds acenaphthene- $\mathrm{d}_{10}$ and pyrene- $\mathrm{d}_{10}$ was then added. The sample extracts were stored in a freezer at $-20^{\circ} \mathrm{C}$ until analysis (up to 30 days).

\section{Extraction of Pesticides from Suspended Sediment}

Filter papers were cut up and placed in an Erlenmeyer flask, spiked with the recovery surrogate standards $\mathrm{d}_{14}$-trifluralin, ${ }^{13} \mathrm{C}_{12}-p, p$ '-DDE, and ${ }^{13} \mathrm{C}_{6}$-permethrin (Cambridge Isotopes, Andover, Mass.) and extracted twice with $50 \mathrm{~mL}$ of dichloromethane in a sonicator (Branson 5200, Danbury, Conn.) for 5 minutes. The extract was filtered through sodium sulfate, reduced using a Zymark Turbovap II (Hopinkton, Md) to $0.5 \mathrm{~mL}$, then solvent exchanged into EtOAc, and further evaporated to less than $0.2 \mathrm{~mL}$ using a gentle stream of dry nitrogen. The internal standard, a mixture of deuterated compounds acenaphthene- $\mathrm{d}_{10}$ and pyrene- $\mathrm{d}_{10}$ was then added. The sample extracts were stored in a freezer at $-20^{\circ} \mathrm{C}$ until analysis (up to 30 days).

\section{Instrument Methods}

Water extracts for analysis by LC/MS/MS were analyzed on an Agilent (Palo Alto, Calif.) 1260 Infinity coupled to an Agilent 6430 Triple Quad LC/MS with a Zorbax Eclipse XDB-C 18 column (2.1 by 150 by 3.5 millimeters, $\mathrm{mm}$; Agilent). The column flow rate was $0.6 \mathrm{~mL} / \mathrm{min}$, and the column temperature was $30^{\circ} \mathrm{C}$. Data were collected in the multiple-reaction-monitoring mode. Additional details about the instrument method can be found in Hladik and Calhoun (2012). 
Water and filter extracts for analysis by GC/MS were analyzed on an Agilent 7890A gas chromatograph with an Agilent 5975C inert mass-selective detector system using a DB-5MS analytical column ( 30 meter, $\mathrm{m}$, by $0.25 \mathrm{~mm}$ by $0.25 \mu \mathrm{m}$; Agilent) for separation with helium as the carrier gas. Data were collected in the selected ion-monitoring mode. Additional details of the GC/MS method can be found in Hladik and others $(2008,2009)$.

\section{National Water Quality Laboratory, Denver, Colorado}

All samples collected for analysis at the NWQL were processed and preserved at the OCRL in Sacramento, Calif., prior to shipment on ice to the NWQL in Denver, Colo.

\section{Copper Methods}

Water samples for copper analysis were pumped through a $0.45-\mu \mathrm{m}$ capsule filter (Pall Versapor WMV High Capacity) using a peristaltic pump and collected in an acid-rinsed 250-mL high-density polyethylene bottle. Prior to sample collection, the capsule filter was rinsed with $2 \mathrm{~L}$ of ASTM Type-I deionized water followed by $25 \mathrm{~mL}$ of native water; the sample collection bottle was rinsed three times with ASTM Type-I deionized water and once with filtered native water. The sample was then preserved with certified, traceable nitric acid obtained from the NWQL. Copper was analyzed at the NWQL using the method described in Garbarino and others, (2006).

\section{Inorganic Carbon, Organic Carbon, and Nitrogen Methods}

Teflon $^{\text {TM }}$ filter towers with 25-mm filters were used for DOC, PIC, POC, TPC, and TPN samples. Sample water was passed through filters using gravity or compressed air. Water samples for DOC analysis were filtered through baked $0.3-\mu \mathrm{m}$ glass-fiber filters (Advantec, Japan) into precleaned, baked $125-\mathrm{mL}$ amber-glass bottles then preserved with certified, traceable sulfuric acid obtained from the NWQL. Particulate analytes (PIC, POC, TPC, and TPN) were collected on three baked $0.3-\mu \mathrm{m}$ glass-fiber filters (Advantec, Japan) and stored wrapped in aluminum foil. Native water was passed through each filter until the filter appeared to be covered with particulate and the total volume of water that passed through each filter was recorded. Dissolved organic carbon was analyzed at the NWQL using the method described in Brenton and Arnett, (1993). Particulate inorganic carbon, POC, TPC, and TPN were analyzed at the NWQL using U.S. Environmental Protection Agency (EPA) method 440.0 (Zimmerman and others, 1997).

\section{Method Detection Limits and Reporting Levels}

Method detection limits (MDLs) for pesticide concentrations in surface water were validated in previous work (Hladik and others, 2008; Hladik and Calhoun, 2012) using the procedure described in 40 CFR 136, appendix B (U.S. Environmental Protection Agency, 1992). Method detection limits for pesticide compounds in suspended sediments filtered from surface water were validated in previous studies by Hladik and others (2009) and Hladik and McWayne (2012). Method detection limits for pesticide concentrations measured in surface water and suspended sediments are listed in table 2. Analytes can sometimes be identified at concentrations less than the MDLs with lower confidence in the numerical value; therefore, concentrations of compounds detected below the MDLs are reported as estimates and coded with an "E."

Method detection limits for DOC and copper were determined by the NWQL by analyzing a series of spiked replicate samples and entering the results into the DQCALC software package following procedures described in ASTM International's Standard Practice D7510-10 (ASTM International, 2010). The method detection limit determined by DQCALC is the lowest concentration at which the chance of a false positive is equal to or less than 1 percent. Reporting levels are reevaluated annually and subject to change, but the reporting level is generally two times the method detection limit. The reporting level is used to control false negative error.

Method detection limits for PIC, POC, TPC, and TPN were determined by the NWQL using the long-term method detection level (LT-MDL) following protocols described in Childress and others (1999). The LT-MDL is used to limit the chance of reporting false positives. Analytes with positive detections, but concentrations measured below the LT-MDL, were flagged with an "E" result-level qualifier. Results with an "E" qualifier have a high certainty of a positive detection, but the exact concentration is uncertain.

Laboratory reporting levels are used to control false negative error and are usually set at two times the LT-MDL. Particulate organic carbon was reported based on the minimum reporting level (MRL). The MRL is the "smallest measured concentration of a constituent that may be reliably reported using a given analytical method" (Timme, 1995). Results below the MRL were flagged with a " $<$ " qualifier. Reporting levels for the analytes measured at the NWQL in this study are listed in table 3. 
Table 2. Method detection limits for dissolved pesticides in surface water and on suspended sediments measured by the U.S. Geological Survey Organic Chemistry Research Laboratory.

[GC/MS, gas chromatography mass spectrometry; LC/MS/MS, liquid chromatography/tandem mass spectrometry; ng/L, nanogram per liter; NWIS, National Water Information System]

\begin{tabular}{|c|c|c|c|c|c|}
\hline Compound & $\begin{array}{c}\text { NWIS } \\
\text { parameter } \\
\text { code }\end{array}$ & Chemical class & Primary pesticide use & $\begin{array}{c}\text { Method } \\
\text { detection limit } \\
\text { (ng/L) }\end{array}$ & $\begin{array}{c}\text { Analytical } \\
\text { method }\end{array}$ \\
\hline Acetamiprid & 68302 & Neonicotinoid & Insecticide & 3.3 & $\mathrm{LC} / \mathrm{MS} / \mathrm{MS}$ \\
\hline Acibenzolar-S-methyl & 51849 & Unclassified & Fungicide & 3.0 & $\mathrm{GC} / \mathrm{MS}$ \\
\hline Alachlor & 65064 & Chloroacetanilide & Herbicide & 1.7 & $\mathrm{GC} / \mathrm{MS}$ \\
\hline Allethrin & 66586 & Pyrethroid & Insecticide & 1.0 & GC/MS \\
\hline Azinphos-methyl & 65066 & Organophosphorus & Insecticide & 9.4 & $\mathrm{GC} / \mathrm{MS}$ \\
\hline Azinphos-methyl oxon & 68211 & Organophosphorus & Breakdown product & 9.4 & $\mathrm{GC} / \mathrm{MS}$ \\
\hline Azoxystrobin & 66589 & Strobin & Fungicide & 3.1 & GC/MS \\
\hline Benefin (benfluralin) & 51643 & 2,6-Dinitroaniline & Herbicide & 2.0 & GC/MS \\
\hline Butralin & 68545 & 2,6-Dinitroaniline & Herbicide & 2.6 & GC/MS \\
\hline Butylate & 65068 & Thiocarbamate & Herbicide & 1.8 & $\mathrm{GC} / \mathrm{MS}$ \\
\hline Captan & 68322 & Thiophthalimide & Fungicide & 10.2 & GC/MS \\
\hline Carbaryl & 65069 & $N$-Methyl carbamate & Insecticide & 6.5 & $\mathrm{GC} / \mathrm{MS}$ \\
\hline Carbendazim & 68548 & Benzimidazole & Fungicide & 4.2 & $\mathrm{LC} / \mathrm{MS} / \mathrm{MS}$ \\
\hline Carbofuran & 65070 & $N$-Methyl carbamate & Insecticide & 3.1 & GC/MS \\
\hline Chlorantraniliprole & 51856 & Anthranilic diamide & Insecticide & 4.0 & $\mathrm{LC} / \mathrm{MS} / \mathrm{MS}$ \\
\hline Chlorothalonil & 65071 & Substituted benzene & Fungicide & 4.1 & $\mathrm{GC} / \mathrm{MS}$ \\
\hline Chlorpyrifos & 65072 & Organophosphorus & Insecticide & 2.1 & $\mathrm{GC} / \mathrm{MS}$ \\
\hline Cycloate & 65073 & Thiocarbamate & Herbicide & 1.1 & $\mathrm{GC} / \mathrm{MS}$ \\
\hline Cyfluthrin & 65074 & Pyrethroid & Insecticide & 1.0 & GC/MS \\
\hline Cyhalofop-butyl & 68360 & Aryloxyphenoxy propionic acid & Herbicide & 1.9 & GC/MS \\
\hline Cyhalothrin (all isomers) & 68354 & Pyrethroid & Insecticide & 0.5 & $\mathrm{GC} / \mathrm{MS}$ \\
\hline Cymoxanil & 51861 & Unclassified & Fungicide & 3.9 & LC/MS/MS \\
\hline Cypermethrin & 65075 & Pyrethroid & Insecticide & 1.0 & GC/MS \\
\hline Cyproconazole & 66593 & Azole & Fungicide & 4.7 & $\mathrm{GC} / \mathrm{MS}$ \\
\hline Cyprodinil & 67574 & Pyrimidine & Fungicide & 7.4 & GC/MS \\
\hline DCPA & 65076 & Alkyl phthalate & Herbicide & 2.0 & $\mathrm{GC} / \mathrm{MS}$ \\
\hline DCPMU & 68231 & Urea & Breakdown product & 3.5 & $\mathrm{LC} / \mathrm{MS} / \mathrm{MS}$ \\
\hline DCPU & 68226 & Urea & Breakdown product & 3.4 & $\mathrm{LC} / \mathrm{MS} / \mathrm{MS}$ \\
\hline Deltamethrin & 65077 & Pyrethroid & Insecticide & 0.6 & $\mathrm{GC} / \mathrm{MS}$ \\
\hline Desthio-prothioconazole & 51865 & Unclassified & Breakdown product & 3.0 & $\mathrm{LC} / \mathrm{MS} / \mathrm{MS}$ \\
\hline
\end{tabular}


Table 2. Method detection limits for dissolved pesticides in surface water and on suspended sediments measured by the U.S. Geological Survey Organic Chemistry Research Laboratory.-Continued

[GC/MS, gas chromatography mass spectrometry; LC/MS/MS, liquid chromatography/tandem mass spectrometry; ng/L, nanogram per liter; NWIS, National Water Information System]

\begin{tabular}{|c|c|c|c|c|c|}
\hline Compound & $\begin{array}{c}\text { NWIS } \\
\text { parameter } \\
\text { code }\end{array}$ & Chemical class & Primary pesticide use & $\begin{array}{c}\text { Method } \\
\text { detection limit } \\
\text { (ng/L) }\end{array}$ & $\begin{array}{c}\text { Analytical } \\
\text { method }\end{array}$ \\
\hline Desulfinylfipronil & 66607 & Unclassified & Breakdown product & 1.6 & GC/MS \\
\hline Desulfinylfipronil amide & 68570 & Unclassified & Breakdown product & 3.2 & GC/MS \\
\hline Diazinon & 65078 & Organophosphorus & Insecticide & 0.9 & GC/MS \\
\hline Diazoxon & 68236 & Organophosphorus & Breakdown product & 5.0 & GC/MS \\
\hline 3,5-Dichloroaniline & 67536 & Unclassified & Breakdown product & 7.6 & $\mathrm{GC} / \mathrm{MS}$ \\
\hline Difenoconazole & 67582 & Azole & Fungicide & 10.5 & GC/MS \\
\hline Dimethomorph & 68373 & Morpholine & Fungicide & 6.0 & GC/MS \\
\hline Dinotefuran & 68379 & Neonicotinoid & Insecticide & 4.5 & $\mathrm{LC} / \mathrm{MS} / \mathrm{MS}$ \\
\hline Esfenvalerate & 65081 & Pyrethroid & Insecticide & 0.5 & GC/MS \\
\hline Ethaboxam & 51855 & Unclassified & Fungicide & 3.8 & $\mathrm{LC} / \mathrm{MS} / \mathrm{MS}$ \\
\hline Ethalfluralin & 65082 & 2,6-Dinitroaniline & Herbicide & 3.0 & $\mathrm{GC} / \mathrm{MS}$ \\
\hline Etofenprox & 67604 & Pyrethroid ether & Insecticide & 2.2 & GC/MS \\
\hline Famoxadone & 67609 & Oxazolidinedione & Fungicide & 2.5 & GC/MS \\
\hline Fenamidone & 51848 & Imidazole & Fungicide & 5.1 & GC/MS \\
\hline Fenarimol & 67613 & Pyrimidine & Fungicide & 6.5 & GC/MS \\
\hline Fenbuconazole & 67618 & Azole & Fungicide & 5.2 & GC/MS \\
\hline Fenhexamid & 67622 & Anilide & Fungicide & 7.6 & $\mathrm{GC} / \mathrm{MS}$ \\
\hline Flonicamid & 51858 & Unclassified & Insecticide & 3.4 & $\mathrm{LC} / \mathrm{MS} / \mathrm{MS}$ \\
\hline Fluazinam & 67636 & 2,6-Dinitroaniline & Fungicide & 4.4 & GC/MS \\
\hline Fludioxonil & 67640 & Unclassified & Fungicide & 7.3 & GC/MS \\
\hline Flufenacet & 51840 & Anilide & Herbicide & 4.7 & GC/MS \\
\hline Flumetralin & 51841 & 2,6-Dinitroaniline & Plant growth regulator & 5.8 & GC/MS \\
\hline Fluopicolide & 51852 & Benzamide pyridine & Fungicide & 3.9 & GC/MS \\
\hline Fluoxastrobin & 67645 & Strobin & Fungicide & 9.5 & GC/MS \\
\hline Fluridone & 51864 & Unclassified & Herbicide & 3.7 & $\mathrm{LC} / \mathrm{MS} / \mathrm{MS}$ \\
\hline Flusilazole & 67649 & Azole & Fungicide & 4.5 & $\mathrm{GC} / \mathrm{MS}$ \\
\hline Flutolanil & 51842 & Anilide & Fungicide & 4.4 & GC/MS \\
\hline Flutriafol & 67653 & Azole & Fungicide & 4.2 & GC/MS \\
\hline Fluxapyroxad & 51851 & Anilide, pyrazole & Fungicide & 4.8 & GC/MS \\
\hline Hexazinone & 65085 & Triazinone & Herbicide & 8.4 & GC/MS \\
\hline
\end{tabular}


Table 2. Method detection limits for dissolved pesticides in surface water and on suspended sediments measured by the U.S. Geological Survey Organic Chemistry Research Laboratory.-Continued

[GC/MS, gas chromatography mass spectrometry; LC/MS/MS, liquid chromatography/tandem mass spectrometry; ng/L, nanogram per liter; NWIS, National Water Information System]

\begin{tabular}{|c|c|c|c|c|c|}
\hline Compound & $\begin{array}{c}\text { NWIS } \\
\text { parameter } \\
\text { code }\end{array}$ & Chemical class & Primary pesticide use & $\begin{array}{c}\text { Method } \\
\text { detection limit } \\
\text { (ng/L) }\end{array}$ & $\begin{array}{c}\text { Analytical } \\
\text { method }\end{array}$ \\
\hline Imazalil & 67662 & Azole & Fungicide & 10.5 & GC/MS \\
\hline Imidacloprid & 68426 & Neonicotinoid & Insecticide & 3.8 & LC/MS/MS \\
\hline Indoxacarb & 68627 & Unclassified & Insecticide & 4.9 & GC/MS \\
\hline Ipconazole & 52762 & Azole & Fungicide & 7.8 & $\mathrm{GC} / \mathrm{MS}$ \\
\hline Kresoxim-methyl & 67670 & Strobin & Fungicide & 4.0 & $\mathrm{GC} / \mathrm{MS}$ \\
\hline Malaoxon & 68240 & Organophosphorus & Breakdown product & 5.0 & GC/MS \\
\hline Malathion & 65087 & Organophosphorus & Insecticide & 3.7 & GC/MS \\
\hline Mandipropamid & 51854 & Amide & Fungicide & 3.3 & $\mathrm{LC} / \mathrm{MS} / \mathrm{MS}$ \\
\hline Methoprene & 66623 & Juvenile hormone mimic & Insect growth regulator & 6.4 & $\mathrm{GC} / \mathrm{MS}$ \\
\hline Methoxyfenozide & 68647 & Diacylhydrazine & Insecticide & 2.7 & $\mathrm{LC} / \mathrm{MS} / \mathrm{MS}$ \\
\hline Methyl parathion & 65089 & Organophosphorus & Insecticide & 3.4 & GC/MS \\
\hline Metolachlor & 65090 & Chloroacetanilide & Herbicide & 1.5 & $\mathrm{GC} / \mathrm{MS}$ \\
\hline Molinate & 65091 & Thiocarbamate & Herbicide & 3.2 & $\mathrm{GC} / \mathrm{MS}$ \\
\hline Myclobutanil & 66632 & Azole & Fungicide & 6.0 & $\mathrm{GC} / \mathrm{MS}$ \\
\hline Napropamide & 65092 & Amide & Herbicide & 8.2 & GC/MS \\
\hline Novaluron & 68655 & Benzoylurea & Herbicide & 2.9 & GC/MS \\
\hline Oryzalin & 68663 & 2,6-Dinitroaniline & Herbicide & 5.0 & $\mathrm{LC} / \mathrm{MS} / \mathrm{MS}$ \\
\hline Pebulate & 65097 & Thiocarbamate & Herbicide & 2.3 & $\mathrm{GC} / \mathrm{MS}$ \\
\hline Pendimethalin & 65098 & 2,6-Dinitroaniline & Herbicide & 2.3 & GC/MS \\
\hline Penoxsulam & 51863 & Triazolopyrimidine & Herbicide & 3.5 & $\mathrm{LC} / \mathrm{MS} / \mathrm{MS}$ \\
\hline Pentachloroanisole & 66637 & Organochlorine & Breakdown product & 4.7 & GC/MS \\
\hline Pentachloronitrobenzene & 66639 & Substituted benzene & Fungicide & 3.1 & $\mathrm{GC} / \mathrm{MS}$ \\
\hline Permethrin & 65099 & Pyrethroid & Insecticide & 0.6 & GC/MS \\
\hline Phenothrin & 65100 & Pyrethroid & Insecticide & 1.0 & GC/MS \\
\hline Phosmet & 65101 & Organophosphorus & Insecticide & 4.4 & $\mathrm{GC} / \mathrm{MS}$ \\
\hline Picoxystrobin & 51850 & Strobin & Fungicide & 4.2 & GC/MS \\
\hline Piperonyl butoxide & 65102 & Unclassified & Synergist & 2.3 & GC/MS \\
\hline Prodiamine & 51844 & 2,6-Dinitroaniline & Herbicide & 5.2 & $\mathrm{GC} / \mathrm{MS}$ \\
\hline Prometon & 67702 & Triazine & Herbicide & 2.5 & GC/MS \\
\hline Prometryn & 65103 & Triazine & Herbicide & 1.8 & GC/MS \\
\hline
\end{tabular}


Table 2. Method detection limits for dissolved pesticides in surface water and on suspended sediments measured by the U.S. Geological Survey Organic Chemistry Research Laboratory.-Continued

[GC/MS, gas chromatography mass spectrometry; LC/MS/MS, liquid chromatography/tandem mass spectrometry; ng/L, nanogram per liter; NWIS, National Water Information System]

\begin{tabular}{|c|c|c|c|c|c|}
\hline Compound & $\begin{array}{c}\text { NWIS } \\
\text { parameter } \\
\text { code }\end{array}$ & Chemical class & Primary pesticide use & $\begin{array}{c}\text { Method } \\
\text { detection limit } \\
\text { (ng/L) }\end{array}$ & $\begin{array}{c}\text { Analytical } \\
\text { method }\end{array}$ \\
\hline Propanil & 66641 & Anilide & Herbicide & 10.1 & GC/MS \\
\hline Propargite & 68677 & Unclassified & Insecticide & 6.1 & GC/MS \\
\hline Propiconazole & 66643 & Azole & Fungicide & 5.0 & GC/MS \\
\hline Propyzamide & 67706 & Amide & Herbicide & 5.0 & $\mathrm{GC} / \mathrm{MS}$ \\
\hline Pyridaben & 68682 & Unclassified & Insecticide & 5.4 & GC/MS \\
\hline Pyrimethanil & 67717 & Pyrimidine & Fungicide & 4.1 & $\mathrm{GC} / \mathrm{MS}$ \\
\hline Quinoxyfen & 51847 & Quinoline & Fungicide & 3.3 & GC/MS \\
\hline Resmethrin & 65104 & Pyrethroid & Insecticide & 1.0 & GC/MS \\
\hline Tebuconazole & 66649 & Azole & Fungicide & 3.7 & GC/MS \\
\hline Tebupirimfos & 68693 & Organophosphorus & Insecticide & 1.9 & GC/MS \\
\hline Tebupirimfos oxon & 68694 & Organophosphorus & Breakdown product & 2.8 & GC/MS \\
\hline Tefluthrin & 67731 & Pyrethroid & Insecticide & 0.6 & $\mathrm{GC} / \mathrm{MS}$ \\
\hline Tetraconazole & 66654 & Azole & Fungicide & 5.6 & GC/MS \\
\hline Tetradifon & 51651 & Unclassified & Insecticide & 3.8 & $\mathrm{GC} / \mathrm{MS}$ \\
\hline Tetramethrin & 66657 & Pyrethroid & Insecticide & 0.5 & $\mathrm{GC} / \mathrm{MS}$ \\
\hline Thiabendazole & 67161 & Benzimidazole & Fungicide & 3.6 & $\mathrm{LC} / \mathrm{MS} / \mathrm{MS}$ \\
\hline Thiacloprid & 68485 & Neonicotinoid & Insecticide & 3.2 & $\mathrm{LC} / \mathrm{MS} / \mathrm{MS}$ \\
\hline Triallate & 68710 & Thiocarbamate & Herbicide & 2.4 & GC/MS \\
\hline Tribufos & 68711 & Organophosphorus & Defoliant & 3.1 & GC/MS \\
\hline Trifloxystrobin & 66660 & Strobin & Fungicide & 4.7 & $\mathrm{GC} / \mathrm{MS}$ \\
\hline Triflumizole & 67753 & Azole & Fungicide & 6.1 & GC/MS \\
\hline Trifluralin & 65108 & 2,6-Dinitroaniline & Herbicide & 2.1 & GC/MS \\
\hline Triticonazole & 67758 & Azole & Fungicide & 6.9 & $\mathrm{GC} / \mathrm{MS}$ \\
\hline Zoxamide & 67768 & Amide & Fungicide & 3.5 & GC/MS \\
\hline
\end{tabular}


Table 3. Reporting levels for dissolved and suspended consituents measured by the U.S. Geological Survey National Water Quality Laboratory.

[dl-dqc, detection limit by DQCALC software; lt-mdl, long term-method detection levels; mg/L, milligram per liter; mrl, minimum reporting level; NWIS, National Water Information System; $\mu \mathrm{g} / \mathrm{L}$, microgram per liter]

\begin{tabular}{llclccc}
\hline \multicolumn{1}{c}{ Analyte } & \multicolumn{1}{c}{ Group } & $\begin{array}{c}\text { NWIS } \\
\text { parameter code }\end{array}$ & Sample fraction & Reporting level & $\begin{array}{c}\text { Reporting } \\
\text { threshold type }\end{array}$ & Parameter unit \\
\hline Organic carbon & Organics, other & 00681 & Dissolved & 0.23 & $\mathrm{dl}-\mathrm{dqc}$ & $\mathrm{mg} / \mathrm{L}$ \\
Inorganic carbon & Inorganics, major, non-metals & 00688 & Suspended & 0.03 & lt-mdl & $\mathrm{mg} / \mathrm{L}$ \\
Organic carbon & Organics, other & 00689 & Suspended & 0.05 & $\mathrm{mrl}$ & $\mathrm{mg} / \mathrm{L}$ \\
Total carbon & Inorganics, major, non-metals & 00694 & Suspended & 0.05 & $1 \mathrm{lt}-\mathrm{mdl}$ & $\mathrm{mg} / \mathrm{L}$ \\
Copper & Inorganics, minor, metals & 01040 & Dissolved & 0.80 & $\mathrm{dl}-\mathrm{dqc}$ & $\mu \mathrm{g} / \mathrm{L}$ \\
Nitrogen & Nutrient & 49570 & Suspended & 0.030 & lt-mdl & $\mathrm{mg} / \mathrm{L}$ \\
\hline
\end{tabular}

\section{Quality-Control Methods and Results}

A quality assurance program plan (QAPP) was designed by the Aquatic Science Center and approved by the Delta RMP Technical Advisory Committee to ensure data quality (Aquatic Science Center, 2016). Field replicates, field blanks, laboratory matrix spikes, and matrix-spike replicates were used to validate pesticide concentrations measured in the water using GC/MS and LC/MS/MS and in the suspended sediments using GC/MS. Field replicates and blanks were collected and analyzed to validate results for analytes measured at the NWQL.

Pesticide Surrogate Compounds - To assess the efficiency of water sample extraction for the GC/MS and LC/MS/MS analytical methods, ${ }^{13} \mathrm{C}_{3}$-atrazine and $\mathrm{d}_{14}$-trifluralin, and monuron and imidacloprid- $\mathrm{d}_{4}$, respectively, were used as recovery surrogates for extracts. Mean (plus or minus, \pm , standard deviation) recoveries of ${ }^{13} \mathrm{C}_{3}$-atrazine, $\mathrm{d}_{14}$-trifluralin, monuron, and imidacloprid- $\mathrm{d}_{4}$ were $90 \pm 11$ percent, $89 \pm 14$ percent, $83 \pm 12$ percent, and $91 \pm 13$ percent, respectively. To assess the efficiency of filter sample extraction, $\mathrm{d}_{14}$-trifluralin, ${ }^{13} \mathrm{C}_{12}-p$, $p$ '-DDE, and ${ }^{13} \mathrm{C}_{6}$-permethrin were used as recovery surrogates for extracts. Mean $( \pm$ standard deviation) recoveries of $\mathrm{d}_{14}$-trifluralin, ${ }^{13} \mathrm{C}_{12}-p, p$ '-DDE, and ${ }^{13} \mathrm{C}_{6}$-permethrin were $91 \pm 11$ percent, $91 \pm 7$ percent, and $96 \pm 11$ percent, respectively.

Blanks - Six pesticide field blanks (three for analysis by GC/MS, three for analysis by LC/MS/MS) were collected to verify the cleanliness of pesticide sample collection and processing protocols. Filters from the three pesticide field blanks collected for analysis by GC/MS also were saved and analyzed as suspended-sediment field blanks. No pesticides were detected in any of the pesticide field blanks. Six field blanks (three for copper analysis, three for DOC analysis) were collected for analysis at the USGS NWQL. There were no detections in the copper samples. One DOC blank had a detection at 0.37 milligram per liter $(\mathrm{mg} / \mathrm{L}$; reporting level $0.23 \mathrm{mg} / \mathrm{L}$ ).

Replicates-Seven pesticide field-replicate samples (three for analysis by GC/MS, four for analysis by LC/MS/MS) were collected concurrently to test the reproducibility of results based on field sampling methods (tables 4 and 5, respectively). The relative standard deviations (RSD) of all environmental and replicate pairs fell below the control limit of 25 percent. Filters from the three replicate samples were collected as suspended-sediment replicates; there were no pesticide detections in any of the environmental and replicate suspended-sediment pairs. Six field replicates were collected (three for copper analysis, three for DOC analysis) for analysis at the USGS NWQL. Results from the six replicates analyzed by the NWQL met the QAPP data-quality objective of less than 25 percent RSD (U.S. Geological Survey, 2017).

Matrix Spikes-Nine pesticide matrix-spike samples (four for analysis by GC/MS, five for analysis by LC/MS/MS) and six pesticide matrix-spike replicate samples (three for analysis by $\mathrm{GC} / \mathrm{MS}$, three for analysis by LC/MS/MS) were collected to assess pesticide recovery, degradation, sorption, and potential interferences caused by the sampling matrix. The minimum, maximum, and median recoveries and standard deviation of the recoveries are presented in table 6. Recoveries of 151 matrix-spike compounds satisfied the QAPP objectives of 70-130 percent recovery of pesticide matrix-spike compounds. Recoveries of fenthion in 2 samples, mandipropamid in 1 sample, and tebupirimfos oxon in 1 sample exceeded 130 percent recovery of pesticide matrix-spike compounds. All pesticide matrix-spike samples with matrix-spike replicate pairs satisfied the QAPP objective of less than 25 percent relative percent difference between matrix spike and matrix-spike replicate pairs. 
Table 4. Pesticide concentrations with measured detections by gas chromatography with mass spectrometry (GC/MS) in environmental and field replicate water samples collected in the Sacramento-San Joaquin Delta, 2015-16.

[Numbers in brackets are U.S. Geological Survey (USGS) National Water Information System (NWIS) parameter codes. Concentrations are in nanograms per liter. Results in parenthesis ( ) are below method detection limits and are estimates. Abbreviations: E, estimated; h.

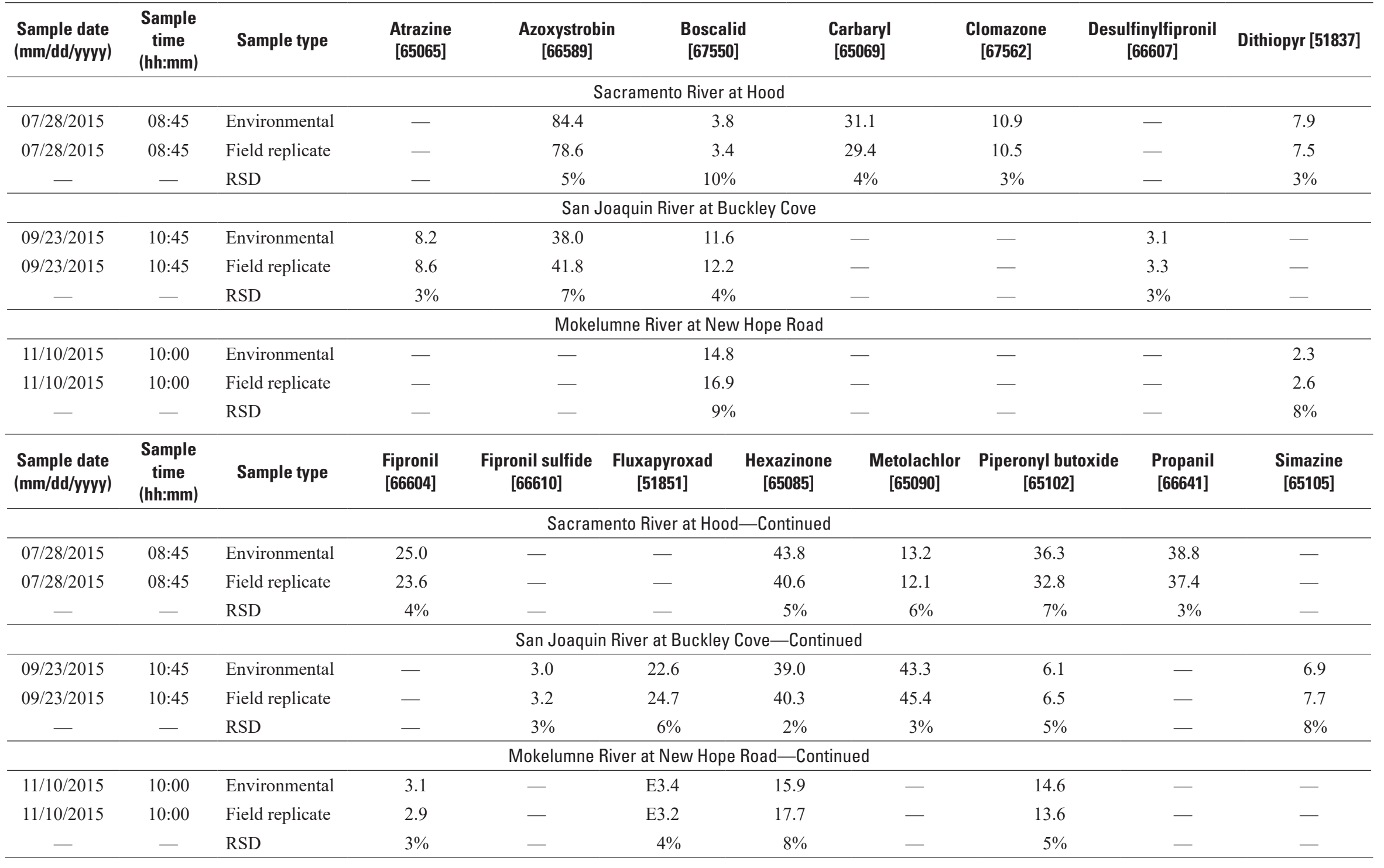


Table 5. Pesticide concentrations with measured detections by liquid chromatography tandem mass spectrometry (LC/MS/MS) in environmental and field replicate water samples collected in the Sacramento-San Joaquin Delta, 2015-16.

[Numbers in brackets are U.S. Geological Survey (USGS) National Water Information System (NWIS) parameter codes. Concentrations are in nanograms per liter. Results in parenthesis ( ) are below method detection limits and are estimates. Abbreviations: E, estimated; hh:mm, hour:minute; mm/dd/yyyy, month/day/year; RSD, relative standard deviation; \%, percent; —, not detected]

\begin{tabular}{|c|c|c|c|c|c|c|c|c|c|c|c|c|}
\hline $\begin{array}{l}\text { Sample date } \\
\text { (mm/dd/yyyy) }\end{array}$ & $\begin{array}{l}\text { Sample time } \\
\text { (hh:mm) }\end{array}$ & Sample type & $\begin{array}{c}\text { Carbendazim } \\
\text { [68548] }\end{array}$ & $\begin{array}{l}\text { Chlor-antranilip- } \\
\text { role } \\
{[51856]}\end{array}$ & $\begin{array}{l}\text { DCPMU } \\
\text { [68231] }\end{array}$ & $\begin{array}{c}\text { DCPU } \\
\text { [68226] }\end{array}$ & $\begin{array}{c}\text { 3,4-Dichloroani- } \\
\text { line } \\
{[66584]}\end{array}$ & $\begin{array}{l}\text { Diuron } \\
{[66598]}\end{array}$ & $\begin{array}{c}\text { Fluridone } \\
\text { [51864] }\end{array}$ & $\begin{array}{c}\text { Imidacloprid } \\
\text { [68426] }\end{array}$ & $\begin{array}{c}\text { Methoxyfenozide } \\
\text { [68647] }\end{array}$ & $\begin{array}{c}\text { Thiabendazole } \\
\text { [67161] }\end{array}$ \\
\hline \multicolumn{13}{|c|}{ San Joaquin River at Buckley Cove } \\
\hline $07 / 28 / 2015$ & 08:45 & Environmental & 61.0 & 5.0 & 49.5 & 9.1 & 9.3 & 86.5 & 382.7 & E3.2 & 47.0 & - \\
\hline $07 / 28 / 2015$ & $08: 45$ & Field replicate & 67.9 & 5.5 & 52.1 & 9.8 & 8.9 & 87.7 & 409.7 & E3.5 & 49.1 & - \\
\hline- & - & RSD & $8 \%$ & $6 \%$ & $4 \%$ & $5 \%$ & $4 \%$ & $1 \%$ & $5 \%$ & $6 \%$ & $3 \%$ & - \\
\hline \multicolumn{13}{|c|}{ Ulatis Creek at Browns Road } \\
\hline $09 / 23 / 2015$ & $14: 15$ & Environmental & - & 36.1 & 4.0 & - & E2.1 & 5.1 & 6.7 & 4.6 & 5.3 & E2.7 \\
\hline $09 / 23 / 2015$ & $14: 15$ & Field replicate & - & 42.5 & 4.8 & - & E2.4 & 5.7 & 7.6 & 5.5 & 6.1 & E3.0 \\
\hline - & - & RSD & - & $12 \%$ & $13 \%$ & - & $9 \%$ & $7 \%$ & $9 \%$ & $12 \%$ & $10 \%$ & $7 \%$ \\
\hline \multicolumn{13}{|c|}{ Sacramento River at Hood } \\
\hline $11 / 10 / 2015$ & 10:00 & Environmental & 21.1 & - & - & - & 3.2 & - & - & - & - & - \\
\hline $11 / 10 / 2015$ & $10: 00$ & Field replicate & 22.2 & - & - & - & 3.3 & - & - & - & - & - \\
\hline - & - & RSD & $4 \%$ & - & - & - & $2 \%$ & - & - & - & - & - \\
\hline \multicolumn{13}{|c|}{ San Joaquin River near Vernalis } \\
\hline $05 / 18 / 2016$ & $12: 20$ & Environmental & - & - & - & - & - & 7.5 & - & - & 7.6 & - \\
\hline $05 / 18 / 2016$ & $12: 20$ & Field replicate & - & - & - & - & - & 8.0 & - & - & 8.1 & - \\
\hline- & - & RSD & - & - & - & - & - & $4 \%$ & - & - & $4 \%$ & - \\
\hline
\end{tabular}


Table 6. Minimum, maximum, and median recovery of compounds in pesticide matrix-spiked water samples with standard deviation.

[Four spiked samples and three spiked replicate samples were analyzed by gas chromatography/mass spectrometry. Compounds noted with ** were analyzed in five spiked samples and three spiked replicate samples by liquid chromatography/tandem mass spectrometry. Abbreviation: \pm , plus or minus]

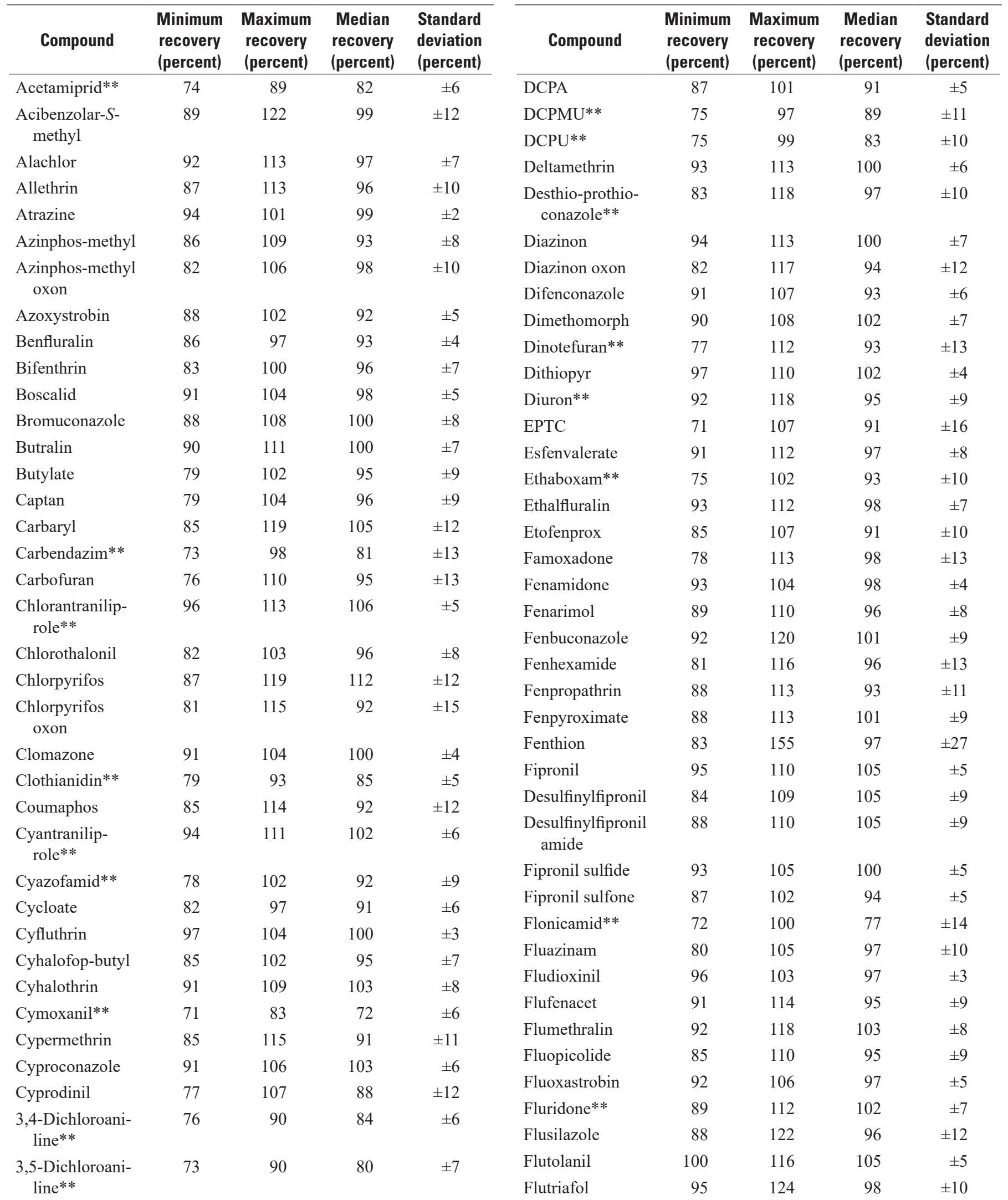


Table 6. Minimum, maximum, and median recovery of compounds in pesticide matrix-spiked water samples with standard deviation.Continued

[Four spiked samples and three spiked replicate samples were analyzed by gas chromatography/mass spectrometry. Compounds noted with ** were analyzed in five spiked samples and three spiked replicate samples by liquid chromatography/tandem mass spectrometry. Abbreviation: \pm , plus or minus]

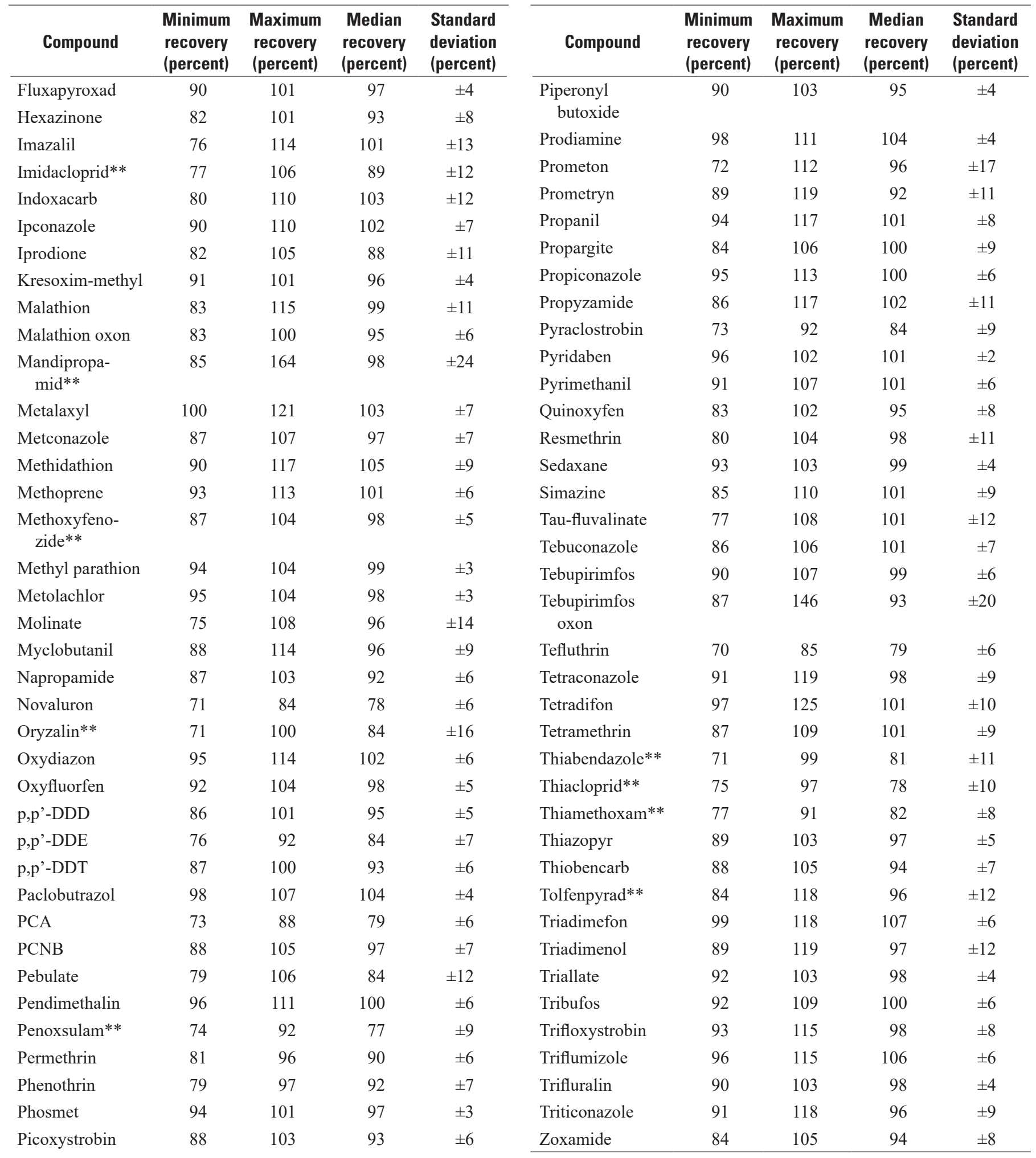




\section{Results}

A total of 54 out of 154 pesticide compounds were detected in water samples collected during the study, and all samples contained multiple pesticides (table 7). A variety of pesticide types were detected in the water during the study period (19 fungicides, 18 herbicides, 9 insecticides, 7 breakdown products, and 1 synergist). The most frequently detected compounds were the herbicide hexazinone ( 95 percent) and the fungicides boscalid (93 percent) and azoxystrobin (75 percent). Overall, 14 pesticides were detected in at least half of all samples. Pesticide concentrations ranged from below the MDLs to 2,630 nanograms per liter (ng/L) for the herbicide metolachlor.

Pesticide concentrations measured in suspended sediments filtered from surface water are presented in table 8. Pesticide concentrations in suspended-sediments filtered from 1-liter water samples are presented using surface water parameter codes and units to facilitate the approximation of a whole-water pesticide concentration (table 9). A total of 11 pesticide compounds were detected in the suspended sediments during the study period (6 herbicides, 3 insecticides, 1 fungicide, and 1 breakdown product). Overall, the most frequently detected pesticides in suspended sediments were the insecticides permethrin ( 7 percent) and bifenthrin ( 5 percent) and the herbicide pendimethalin (5 percent). Pesticide concentrations in the suspended sediments ranged from below the MDLs to $265 \mathrm{ng} / \mathrm{L}$ for the herbicide pendimethalin. Whole-water pesticide concentrations were calculated for compounds with at least one suspended-sediment detection (table 9).

Results for dissolved and suspended constituents measured at the NWQL are provided in table 10 and water quality measurements taken in the field are provided in table 11. Dissolved organic carbon was detected at concentrations from 1.59 to $11.6 \mathrm{mg} / \mathrm{L}$. Particulate inorganic carbon was detected above the MRL of $0.03 \mathrm{mg} / \mathrm{L}$ in five samples ( 8 percent) at concentrations from 0.03 to $0.14 \mathrm{mg} / \mathrm{L}$. Particulate organic carbon was present in 60 samples (100 percent) at concentrations ranging from less than 0.11 to $38.5 \mathrm{mg} / \mathrm{L}$. Total particulate nitrogen was detected above the LT-MDL of $0.03 \mathrm{mg} / \mathrm{L}$ in 52 samples ( 87 percent) at concentrations from 0.031 to $4.59 \mathrm{mg} / \mathrm{L}$. Dissolved copper was detected above the detection limit of $0.8 \mathrm{mg} / \mathrm{L}$ in 58 samples (97 percent) at concentrations ranging from 0.81 to 4.4 micrograms per liter $(\mu \mathrm{g} / \mathrm{L})$.

The data presented in this report are publicly available in the USGS National Water Information System web interface (U.S. Geological Survey, 2017). Results for this report were retrieved from NWIS and compiled in April 2017.

\section{Mokelumne River at New Hope Road}

A total of 32 pesticides (14 fungicides, 10 herbicides, 4 insecticides, 3 breakdown products, and 1 synergist) were detected in water samples collected at this site; the most frequently detected compounds were boscalid (100 percent) and hexazinone (92 percent). The maximum pesticide concentration measured at this site was $247 \mathrm{ng} / \mathrm{L}$ (simazine) in a sample collected on March 7, 2016. A minimum of 2 (October 21, 2015, May 18, 2016, and June 15, 2016) and a maximum of 13 (March 7, 2016) pesticides or pesticide degradates were detected in each water sample (fig. 3). No pesticides or pesticide degradates were detected in the suspended sediments collected at this site. There were no pesticide detections at concentrations above EPA aquatic-life benchmarks at this site (U.S. Environmental Protection Agency, 2017).

\section{Sacramento River at Hood}

A total of 33 pesticides (11 herbicides, 11 fungicides, 6 insecticides, 4 breakdown products, and 1 synergist) were detected in the water samples collected at this site. The fungicide azoxystrobin was detected in every sample, and an additional seven pesticides (hexazinone, 3,4-dichloroaniline, boscalid, diuron, piperonyl butoxide, carbendazim, and fipronil) were detected in at least half of the samples collected at this site. The maximum pesticide concentration measured at this site was $348 \mathrm{ng} / \mathrm{L}$ for azoxystrobin in the sample collected on August 18, 2015. A minimum of 6 (February 17, 2016) and a maximum of 15 (March 7, 2016) pesticides or pesticide degradates were detected in each water sample (fig. 3). Permethrin was detected in the suspended sediments of the sample collected on March 7, 2016, at a concentration of $19.2 \mathrm{ng} / \mathrm{L}$. This was the only detection of pesticides on suspended sediments from this site.

Bifenthrin was detected in the water sample collected on August 18, 2015 (2.9 ng/L), at a concentration above the aquatic-life benchmark for chronic toxicity to invertebrates of 1.3 ng/L (U.S. Environmental Protection Agency, 2017). The insecticide fipronil was detected at concentrations above the aquatic-life benchmark for chronic toxicity to invertebrates of $11 \mathrm{ng} / \mathrm{L}$ in the samples collected on July 28, 2015 (25 ng/L), and November 10, 2015 (12.2 ng/L). 
Table 7. Pesticide concentrations with measured detections in environmental water samples collected in the Sacramento-San Joaquin Delta, 2015-16.

[Numbers in brackets are U.S. Geological Survey (USGS) National Water Information System (NWIS) parameter codes. Concentrations are in nanograms per liter. Abbreviations: E, estimated; hh:mm, hour:minute; mm/dd/yyyy, month/day/year; —, not detected; *, storm sample]

\begin{tabular}{|c|c|c|c|c|c|c|c|c|c|c|c|c|}
\hline $\begin{array}{l}\text { Sample date } \\
\text { (mm/dd/yyyy) }\end{array}$ & $\begin{array}{l}\text { Sample } \\
\text { time } \\
\text { (hh:mm) }\end{array}$ & $\begin{array}{c}\text { Acibenzolar- } \\
\text { S-methyl } \\
\text { [51849] }\end{array}$ & $\begin{array}{c}\text { Atrazine } \\
\text { [65065] }\end{array}$ & $\begin{array}{c}\text { Azoxystrobin } \\
\text { [66589] }\end{array}$ & $\begin{array}{l}\text { Bifenthrin } \\
\text { [65067] }\end{array}$ & $\begin{array}{l}\text { Boscalid } \\
\text { [67550] }\end{array}$ & $\begin{array}{c}\text { Carbaryl } \\
\text { [65069] }\end{array}$ & $\begin{array}{l}\text { Carbendazim } \\
\text { [68548] }\end{array}$ & $\begin{array}{c}\text { Chlorantraniliprole } \\
\text { [51856] }\end{array}$ & $\begin{array}{c}\text { Chlorothalonil } \\
\text { [65071] }\end{array}$ & $\begin{array}{l}\text { Clomazone } \\
\text { [67562] }\end{array}$ & $\begin{array}{l}\text { Cyprodinil } \\
\text { [67574] }\end{array}$ \\
\hline \multicolumn{13}{|c|}{ Mokelumne River at New Hope Road } \\
\hline $07 / 28 / 2015$ & 09:50 & - & - & 54.4 & - & 10.4 & - & - & - & - & 11.9 & - \\
\hline $08 / 18 / 2015$ & 09:50 & - & - & 115 & - & 21.2 & - & - & - & - & - & - \\
\hline $09 / 23 / 2015$ & $09: 20$ & - & - & 90.1 & - & 3.5 & - & 4.3 & - & E2.3 & - & - \\
\hline $10 / 21 / 2015$ & $09: 10$ & - & - & - & - & 11.1 & - & - & - & - & - & - \\
\hline $11 / 10 / 2015$ & 10:00 & - & - & - & - & 14.8 & - & E3.0 & - & - & - & - \\
\hline $12 / 15 / 2015$ & $14: 00$ & - & - & - & - & E2.5 & - & 11.6 & - & - & - & - \\
\hline$* 01 / 19 / 2016$ & $09: 45$ & - & - & - & - & 9.2 & - & E3.1 & E3.7 & - & - & - \\
\hline $02 / 17 / 2016$ & 09:00 & - & - & - & - & 4.8 & - & - & - & - & - & - \\
\hline *03/07/2016 & $13: 30$ & - & - & - & - & 4.7 & - & 5.6 & - & - & - & - \\
\hline 04/19/2016 & 09:20 & - & - & 14.1 & - & 20.7 & - & - & - & 6.7 & - & 9.1 \\
\hline 05/18/2016 & $09: 30$ & - & - & - & - & 7.3 & - & - & - & - & - & - \\
\hline $06 / 15 / 2016$ & $09: 15$ & - & - & - & - & 6.4 & - & - & - & - & - & - \\
\hline \multicolumn{13}{|c|}{ Sacramento River at Hood } \\
\hline $07 / 28 / 2015$ & 08:45 & - & - & 84.4 & - & 3.8 & 31.1 & - & - & - & 10.9 & - \\
\hline $08 / 18 / 2015$ & 08:40 & - & - & 348 & 2.9 & 7.5 & - & 20.6 & - & - & 4.6 & - \\
\hline $09 / 23 / 2015$ & 08:30 & - & - & 19.2 & - & - & - & 29.4 & - & E2.9 & - & - \\
\hline $10 / 21 / 2015$ & 08:00 & - & - & 35.9 & - & 10.6 & - & 21.1 & - & - & - & - \\
\hline $11 / 10 / 2015$ & 09:00 & - & - & 93.9 & - & 6.3 & - & 37.9 & - & - & - & - \\
\hline $12 / 15 / 2015$ & $15: 00$ & - & - & 30.3 & - & - & - & 18.5 & - & - & - & - \\
\hline *01/19/2016 & $08: 30$ & - & - & 32.6 & - & - & - & 4.2 & 4.4 & - & - & - \\
\hline $02 / 17 / 2016$ & 08:00 & - & - & 28.9 & - & - & - & - & - & - & - & - \\
\hline *03/07/2016 & $14: 20$ & - & - & 7.3 & - & 3 & - & 4.4 & - & - & - & 9.2 \\
\hline $04 / 19 / 2016$ & $08: 20$ & - & - & 15.3 & - & 13.4 & - & - & - & 6.8 & - & - \\
\hline $05 / 18 / 2016$ & $08: 30$ & - & - & 11.8 & - & 7.2 & - & - & - & - & 135 & - \\
\hline $06 / 15 / 2016$ & 08:10 & - & - & 24.5 & - & 8.9 & - & - & - & - & 47.6 & - \\
\hline \multicolumn{13}{|c|}{ San Joaquin River at Buckley Cove } \\
\hline $07 / 28 / 2015$ & $11: 10$ & - & 49.3 & 58.1 & - & 34.1 & - & 61 & 5 & - & 22.9 & - \\
\hline 08/18/2015 & $11: 10$ & - & 21.4 & 118 & - & 46 & - & 58.6 & 9.3 & - & 10.7 & - \\
\hline 09/23/2015 & $10: 45$ & - & 8.2 & 38 & - & 11.6 & - & 70.7 & 10.3 & - & - & - \\
\hline
\end{tabular}


Table 7. Pesticide concentrations with measured detections in environmental water samples collected in the Sacramento-San Joaquin Delta, 2015-16.-Continued

[Numbers in brackets are U.S. Geological Survey (USGS) National Water Information System (NWIS) parameter codes. Concentrations are in nanograms per liter. Abbreviations: E, estimated; hh:mm, hour:minute; mm/dd/yyyy, month/day/year; —, not detected; *, storm sample]

\begin{tabular}{|c|c|c|c|c|c|c|c|c|c|c|c|c|}
\hline $\begin{array}{l}\text { Sample date } \\
\text { (mm/dd/yyyy) }\end{array}$ & $\begin{array}{c}\text { Sample } \\
\text { time } \\
\text { (hh:mm) }\end{array}$ & $\begin{array}{c}\text { Acibenzolar- } \\
\text { S-methyl } \\
\text { [51849] }\end{array}$ & $\begin{array}{c}\text { Atrazine } \\
\text { [65065] }\end{array}$ & $\begin{array}{c}\text { Azoxystrobin } \\
{[66589]}\end{array}$ & $\begin{array}{l}\text { Bifenthrin } \\
\text { [65067] }\end{array}$ & $\begin{array}{l}\text { Boscalid } \\
\text { [67550] }\end{array}$ & $\begin{array}{c}\text { Carbaryl } \\
\text { [65069] }\end{array}$ & $\begin{array}{c}\text { Carbendazim } \\
\text { [68548] }\end{array}$ & $\begin{array}{c}\text { Chlorantraniliprole } \\
\text { [51856] }\end{array}$ & $\begin{array}{c}\text { Chlorothalonil } \\
\text { [65071] }\end{array}$ & $\begin{array}{c}\text { Clomazone } \\
\text { [67562] }\end{array}$ & $\begin{array}{c}\text { Cyprodinil } \\
\text { [67574] }\end{array}$ \\
\hline \multicolumn{13}{|c|}{ San Joaquin River at Buckley Cove-Continued } \\
\hline $10 / 21 / 2015$ & $10: 50$ & - & - & 129 & - & 37.6 & - & 107 & 11 & - & - & - \\
\hline $11 / 10 / 2015$ & $11: 30$ & - & - & 66.7 & - & 28.1 & - & 33.4 & $\mathrm{E} 2.0$ & - & - & - \\
\hline $12 / 15 / 2015$ & $12: 20$ & - & - & 14.1 & - & 6.9 & - & 20.3 & 4.7 & - & - & - \\
\hline *01/19/2016 & $10: 50$ & - & - & 11.9 & - & 118 & - & 50.1 & 21.1 & - & - & - \\
\hline $02 / 17 / 2016$ & $10: 10$ & - & - & 5.6 & - & 67.6 & - & 8.8 & 14.4 & - & - & - \\
\hline$* 03 / 07 / 2016$ & $10: 15$ & - & - & 3.7 & - & 24.7 & - & 35 & 10.6 & - & - & - \\
\hline $04 / 19 / 2016$ & $10: 40$ & 88.4 & - & 48.4 & - & 93.7 & - & - & 8.9 & - & - & - \\
\hline 05/18/2016 & $10: 45$ & - & - & 14.7 & - & 14.6 & - & - & - & - & - & - \\
\hline $06 / 15 / 2016$ & $10: 50$ & - & 40 & 33.2 & - & 28.6 & - & 7.1 & - & - & - & - \\
\hline \multicolumn{13}{|c|}{ San Joaquin River near Vernalis } \\
\hline $07 / 28 / 2015$ & $12: 15$ & - & 9.7 & 5.1 & - & 9.1 & - & - & E3.9 & - & - & - \\
\hline $08 / 18 / 2015$ & $12: 50$ & - & 3.9 & 16 & - & 17.1 & - & - & 4 & - & - & - \\
\hline $09 / 23 / 2015$ & $12: 20$ & - & - & 24.5 & - & 3.7 & - & - & 4.9 & E3.4 & - & - \\
\hline $10 / 21 / 2015$ & $12: 40$ & - & - & 37.7 & - & 13.4 & - & - & 5.1 & - & - & - \\
\hline $11 / 10 / 2015$ & $13: 00$ & - & - & 22.2 & - & 17.9 & - & 9.2 & - & - & - & - \\
\hline $12 / 15 / 2015$ & $11: 00$ & - & - & - & - & 3.8 & - & - & E2.9 & - & - & - \\
\hline *01/19/2016 & $12: 30$ & - & - & 16.5 & - & 39.4 & - & 7.5 & 10.8 & - & - & - \\
\hline $02 / 17 / 2016$ & $12: 00$ & - & - & - & - & 27.5 & - & 7.6 & 7.3 & - & - & 11 \\
\hline *03/07/2016 & $11: 50$ & - & - & 4.2 & - & 21.8 & - & 40.7 & 6.5 & - & - & - \\
\hline $04 / 19 / 2016$ & $12: 45$ & 23.7 & - & 27.9 & - & 49.7 & - & - & 4.1 & - & - & - \\
\hline 05/18/2016 & $12: 20$ & - & - & 12.9 & - & 12.5 & - & - & - & - & - & - \\
\hline $06 / 15 / 2016$ & $12: 40$ & - & - & 14.6 & - & 15.5 & - & - & - & - & - & - \\
\hline \multicolumn{13}{|c|}{ Ulatis Creek at Browns Road } \\
\hline $07 / 28 / 2015$ & $14: 20$ & - & 14.6 & 34.7 & - & 32.6 & - & - & 150 & - & - & - \\
\hline $08 / 18 / 2015$ & $15: 00$ & - & 5.9 & 104 & - & 47.5 & - & - & 36.1 & - & - & - \\
\hline $09 / 23 / 2015$ & $14: 15$ & - & 4.1 & 5.9 & - & 10 & - & - & 49.5 & - & - & - \\
\hline $10 / 21 / 2015$ & $15: 00$ & - & - & 54.6 & - & 47.3 & 20.7 & 156 & 12.3 & - & - & - \\
\hline $11 / 10 / 2015$ & $15: 30$ & - & 14.1 & - & 33.3 & 66.8 & - & - & E2.3 & - & - & - \\
\hline $12 / 15 / 2015$ & $08: 50$ & - & 6.3 & - & 13.2 & 23.5 & - & 57 & E2.4 & - & - & - \\
\hline
\end{tabular}


Table 7. Pesticide concentrations with measured detections in environmental water samples collected in the Sacramento-San Joaquin Delta, 2015-16.-Continued

[Numbers in brackets are U.S. Geological Survey (USGS) National Water Information System (NWIS) parameter codes. Concentrations are in nanograms per liter. Abbreviations: E, estimated; hh:mm, hour:minute; mm/dd/yyyy, month/day/year; —, not detected; *, storm sample]

\begin{tabular}{|c|c|c|c|c|c|c|c|c|c|c|c|c|c|}
\hline $\begin{array}{l}\text { Sample date } \\
\text { (mm/dd/yyyy) }\end{array}$ & $\begin{array}{c}\text { Sample } \\
\text { time } \\
\text { (hh:mm) }\end{array}$ & $\begin{array}{c}\text { Acibenzolar- } \\
\text { S-methyl } \\
{[51849]}\end{array}$ & $\begin{array}{c}\text { Atrazine Azc } \\
\text { [65065] }\end{array}$ & $\begin{array}{l}\text { Azoxystrobin } \\
\text { [66589] }\end{array}$ & $\begin{array}{c}\text { Bifenthrin } \\
\text { [65067] }\end{array}$ & $\begin{array}{c}\text { Boscalid } \\
\text { [67550] }\end{array}$ & $\begin{array}{c}\text { Carbaryl } \\
\text { [65069] }\end{array}$ & $\begin{array}{c}\text { Carbendazim } \\
\text { [68548] }\end{array}$ & $\begin{array}{c}\text { Chlorantraniliprole } \\
{[51856]}\end{array}$ & $\begin{array}{c}\text { Chlorothalonil } \\
\text { [65071] }\end{array}$ & \multicolumn{2}{|c|}{$\begin{array}{c}\text { il Clomazone } \\
{[67562]}\end{array}$} & $\begin{array}{l}\text { Cyprodinil } \\
\text { [67574] }\end{array}$ \\
\hline \multicolumn{14}{|c|}{ Ulatis Creek at Browns Road-Continued } \\
\hline$* 01 / 19 / 2016$ & $15: 00$ & - & - & - & - & 82.1 & - & E4.0 & 25.9 & - & - & & - \\
\hline $02 / 17 / 2016$ & $14: 10$ & - & - & - & 11.5 & 46.2 & - & - & $\mathrm{E} 3.0$ & - & - & & E4.0 \\
\hline *03/07/2016 & $08: 30$ & - & - & 6.5 & - & 64.9 & - & 8 & 26.9 & - & - & & - \\
\hline $04 / 19 / 2016$ & $15: 10$ & - & 10.4 & 29.1 & - & 50.4 & - & - & $\mathrm{E} 2.5$ & - & - & & - \\
\hline $05 / 18 / 2016$ & $14: 20$ & - & - & 14.5 & - & 20.3 & - & - & 14.4 & - & - & & - \\
\hline $06 / 15 / 2016$ & $14: 40$ & - & - & - & - & 25.4 & - & - & 260 & - & - & & - \\
\hline $\begin{array}{l}\text { Sample date } \\
\text { (mm/dd/yyyy) }\end{array}$ & $\begin{array}{c}\text { Sample } \\
\text { time } \\
\text { (hh:mm) }\end{array}$ & $\begin{array}{c}\text { Desulfinylfipronil } \\
\text { [66607] }\end{array}$ & $\begin{array}{c}\text { iil } \begin{array}{c}\text { Diazinon } \\
\text { [65078] }\end{array}\end{array}$ & $\begin{array}{l}\text { 3,4-Dichl } \\
\text { ] }\end{array}$ & $\begin{array}{l}\text { oroaniline } \\
584]\end{array}$ & $\begin{array}{r}\text { 3,5-Dichlor } \\
{[6753}\end{array}$ & $\begin{array}{l}\text { oaniline } \\
\text { 6] }\end{array}$ & $\begin{array}{r}\text { 3,4-Dichlorophe } \\
\text { [68226] }\end{array}$ & $\begin{array}{r}\text { tylurea } \\
N \text {-3,4-Dich } \\
N \text {-me } \\
{[68}\end{array}$ & $\begin{array}{l}\text { Ilorophenyl)- } \\
\text { thylurea } \\
\text { 231] }\end{array}$ & $\begin{array}{l}\text { Jithiopyr } \\
\text { [51837] }\end{array}$ & $\begin{array}{l}\text { Diuron } \\
{[66598]}\end{array}$ & $\begin{array}{c}\text { EPTC } \\
{[65080]}\end{array}$ \\
\hline \multicolumn{14}{|c|}{ Mokelumne River at New Hope Road-Continued } \\
\hline $07 / 28 / 2015$ & 09:50 & 8.1 & - & & 4.7 & - & & - & & - & - & E1.5 & - \\
\hline $08 / 18 / 2015$ & 09:50 & 2.4 & - & & 4.2 & - & & - & & - & - & - & - \\
\hline $09 / 23 / 2015$ & 09:20 & - & - & & 7.6 & - & & - & & - & - & - & - \\
\hline $10 / 21 / 2015$ & 09:10 & - & - & & - & - & & - & & - & - & - & - \\
\hline $11 / 10 / 2015$ & $10: 00$ & - & - & & - & - & & - & & - & 2.3 & - & - \\
\hline $12 / 15 / 2015$ & $14: 00$ & - & - & & - & - & & - & & - & 7 & E2.3 & - \\
\hline *01/19/2016 & 09:45 & - & - & & - & - & & - & & - & 14.8 & 7 & - \\
\hline $02 / 17 / 2016$ & 09:00 & - & - & & - & - & & - & & - & - & E1.8 & - \\
\hline$* 03 / 07 / 2016$ & $13: 30$ & - & 89.1 & & - & - & & - & & 2.6 & 17.4 & 33.7 & - \\
\hline 04/19/2016 & 09:20 & - & - & & - & - & & - & & - & 2.1 & - & - \\
\hline $05 / 18 / 2016$ & 09:30 & - & - & & - & - & & - & & - & - & - & - \\
\hline $06 / 15 / 2016$ & $09: 15$ & - & - & & - & - & & - & & - & - & - & - \\
\hline \multicolumn{14}{|c|}{ Sacramento River at Hood-Continued } \\
\hline $07 / 28 / 2015$ & $08: 45$ & - & - & & 10.5 & - & & - & & - & 7.9 & E1.6 & - \\
\hline $08 / 18 / 2015$ & 08:40 & - & - & & 15.3 & - & & - & & - & - & - & - \\
\hline 09/23/2015 & $08: 30$ & - & - & & 9 & - & & - & & - & - & 4.3 & - \\
\hline $10 / 21 / 2015$ & 08:00 & - & - & & 3.2 & - & & - & & - & - & - & - \\
\hline $11 / 10 / 2015$ & 09:00 & - & - & & 7.8 & - & & E2.6 & & 4.1 & - & 8.3 & - \\
\hline $12 / 15 / 2015$ & $15: 00$ & - & - & & 5.5 & - & & - & & 2.0 & 3.5 & 17.5 & - \\
\hline
\end{tabular}


Table 7. Pesticide concentrations with measured detections in environmental water samples collected in the Sacramento-San Joaquin Delta, 2015-16.-Continued

[Numbers in brackets are U.S. Geological Survey (USGS) National Water Information System (NWIS) parameter codes. Concentrations are in nanograms per liter. Abbreviations: E, estimated; hh:mm, hour:minute; mm/dd/yyyy, month/day/year; —, not detected; *, storm sample]

\begin{tabular}{|c|c|c|c|c|c|c|c|c|c|c|}
\hline $\begin{array}{l}\text { Sample date } \\
\text { (mm/dd/yyyy) }\end{array}$ & $\begin{array}{c}\text { Sample } \\
\text { time } \\
\text { (hh:mm) }\end{array}$ & $\begin{array}{c}\text { Desulfinylfipronil } \\
{[66607]}\end{array}$ & $\begin{array}{l}\text { Diazinon } \\
\text { [65078] }\end{array}$ & $\begin{array}{c}\text { 3,4-Dichloroaniline } \\
\text { [66584] }\end{array}$ & $\begin{array}{c}\text { 3,5-Dichloroaniline } \\
\text { [67536] }\end{array}$ & $\begin{array}{c}\text { 3,4-Dichlorophenylurea } \\
\text { [68226] }\end{array}$ & $\begin{array}{c}N \text {-(3,4-Dichlorophenyl)- } \\
N \text {-methylurea } \\
{[68231]}\end{array}$ & $\begin{array}{l}\text { Dithiopyr } \\
\text { [51837] }\end{array}$ & $\begin{array}{l}\text { Diuron } \\
{[66598]}\end{array}$ & $\begin{array}{l}\text { EPTC } \\
{[65080]}\end{array}$ \\
\hline \multicolumn{11}{|c|}{ Sacramento River at Hood-Continued } \\
\hline$* 01 / 19 / 2016$ & 08:30 & - & - & 5.7 & E1.6 & E2.3 & E2.9 & - & 43 & - \\
\hline $02 / 17 / 2016$ & 08:00 & - & - & - & - & - & $\mathrm{E} 2.0$ & - & 5.2 & - \\
\hline *03/07/2016 & $14: 20$ & - & - & 21.1 & - & $\mathrm{E} 2.2$ & 9.4 & 12 & 134 & - \\
\hline $04 / 19 / 2016$ & 08:20 & - & - & - & - & - & - & - & 4.1 & - \\
\hline $05 / 18 / 2016$ & 08:30 & - & - & E2.6 & - & - & - & - & - & - \\
\hline $06 / 15 / 2016$ & 08:10 & - & - & 145 & - & - & - & - & - & - \\
\hline \multicolumn{11}{|c|}{ San Joaquin River at Buckley Cove-Continued } \\
\hline $07 / 28 / 2015$ & $11: 10$ & 13.1 & - & 9.3 & - & 9.1 & 49.5 & 9.4 & 86.5 & - \\
\hline $08 / 18 / 2015$ & $11: 10$ & 4.9 & - & 6.2 & - & 10.3 & 39.4 & 2.5 & 63.6 & - \\
\hline $09 / 23 / 2015$ & $10: 45$ & 3.1 & - & 4.3 & - & 5.4 & 19.5 & - & 36.5 & - \\
\hline $10 / 21 / 2015$ & $10: 50$ & 4.7 & - & - & - & E2.9 & 8.4 & - & 13.8 & - \\
\hline $11 / 10 / 2015$ & $11: 30$ & 2.1 & - & 3.2 & - & $\mathrm{E} 2.5$ & 3.5 & - & 6.7 & - \\
\hline $12 / 15 / 2015$ & $12: 20$ & 2.7 & - & - & - & - & 4.7 & 2.9 & 27.5 & - \\
\hline$* 01 / 19 / 2016$ & $10: 50$ & - & - & 5 & - & 11.8 & 54.6 & 14.1 & 451 & - \\
\hline $02 / 17 / 2016$ & $10: 10$ & 2.8 & - & 3.9 & - & 9.7 & 26.2 & 22.1 & 158 & - \\
\hline *03/07/2016 & $10: 15$ & - & - & 5.1 & - & 15.9 & 46.4 & 57.5 & 313 & - \\
\hline $04 / 19 / 2016$ & $10: 40$ & - & 14.8 & 3.7 & - & 4.2 & 27.7 & 19.9 & 60.5 & 7.3 \\
\hline 05/18/2016 & $10: 45$ & - & 5.3 & - & - & - & 5.5 & 4 & 10.2 & - \\
\hline $06 / 15 / 2016$ & $10: 50$ & - & - & 53.1 & - & 3.8 & 11.7 & - & 21.3 & 33.7 \\
\hline \multicolumn{11}{|c|}{ San Joaquin River near Vernalis-Continued } \\
\hline $07 / 28 / 2015$ & $12: 15$ & - & - & E1.6 & - & - & E2.4 & 7.2 & 4.3 & - \\
\hline $08 / 18 / 2015$ & $12: 50$ & - & - & - & - & - & - & 1.9 & - & - \\
\hline $09 / 23 / 2015$ & $12: 20$ & - & - & - & - & - & - & - & - & - \\
\hline $10 / 21 / 2015$ & $12: 40$ & - & - & - & - & - & - & - & - & - \\
\hline $11 / 10 / 2015$ & $13: 00$ & - & - & - & - & - & - & 3.1 & - & - \\
\hline $12 / 15 / 2015$ & $11: 00$ & - & - & - & - & - & 8.2 & 14.1 & 26.3 & - \\
\hline$* 01 / 19 / 2016$ & $12: 30$ & - & 7.2 & E3.1 & - & 9.2 & 27.6 & 50.4 & 181 & - \\
\hline $02 / 17 / 2016$ & $12: 00$ & - & - & E3.0 & - & - & 9.9 & 15.2 & 58.3 & - \\
\hline$* 03 / 07 / 2016$ & $11: 50$ & - & - & 3.5 & E3.2 & 8.1 & 21.9 & 51 & 133 & - \\
\hline
\end{tabular}


Table 7. Pesticide concentrations with measured detections in environmental water samples collected in the Sacramento-San Joaquin Delta, 2015-16.-Continued

[Numbers in brackets are U.S. Geological Survey (USGS) National Water Information System (NWIS) parameter codes. Concentrations are in nanograms per liter. Abbreviations: E, estimated; hh:mm, hour:minute; mm/dd/yyyy, month/day/year; —, not detected; *, storm sample]

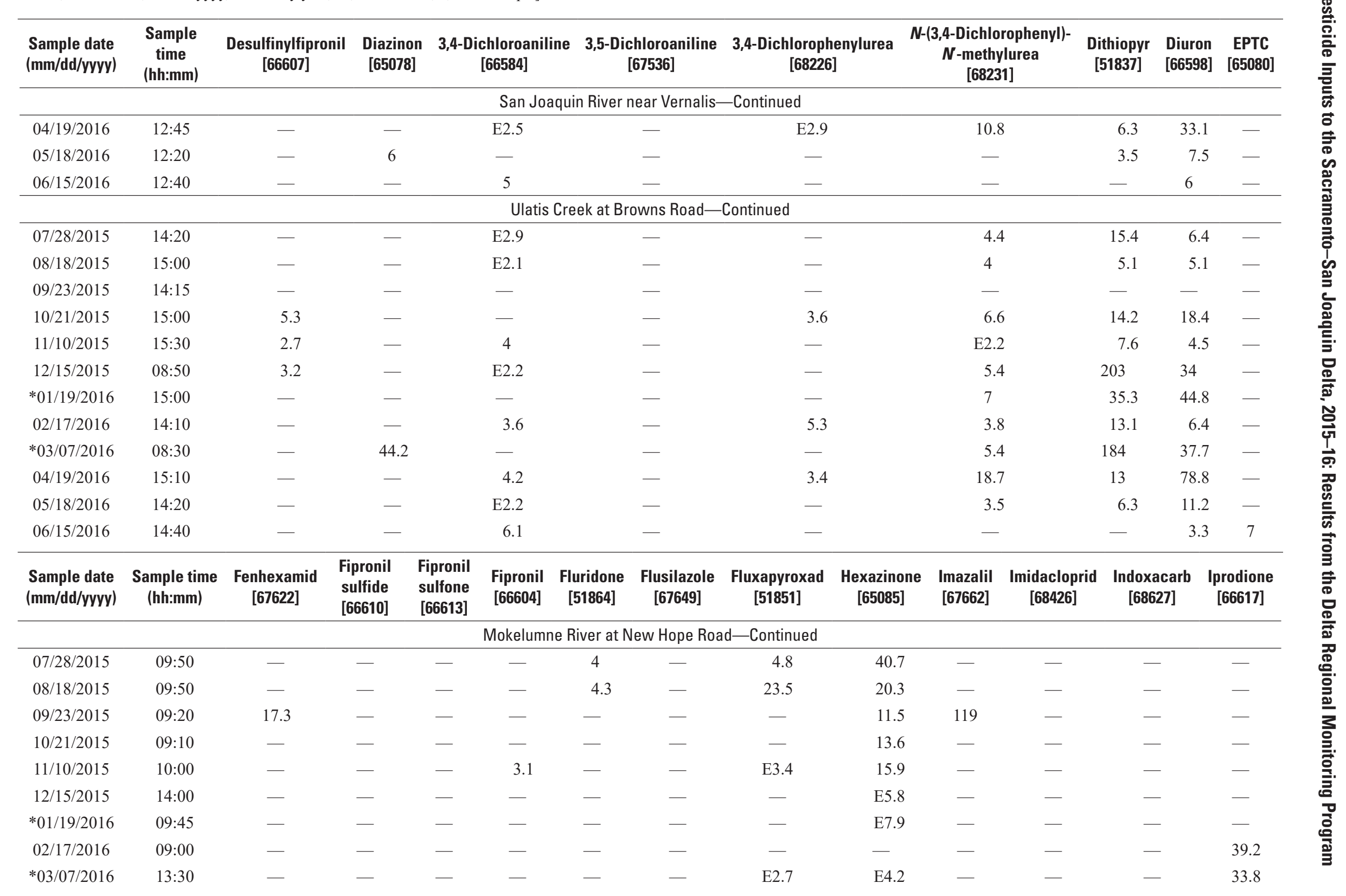


Table 7. Pesticide concentrations with measured detections in environmental water samples collected in the Sacramento-San Joaquin Delta, 2015-16.-Continued

[Numbers in brackets are U.S. Geological Survey (USGS) National Water Information System (NWIS) parameter codes. Concentrations are in nanograms per liter. Abbreviations: E, estimated; hh:mm, hour:minute; mm/dd/yyyy, month/day/year; —, not detected; *, storm sample]

\begin{tabular}{|c|c|c|c|c|c|c|c|c|c|c|c|c|c|}
\hline $\begin{array}{l}\text { Sample date } \\
\text { (mm/dd/yyyy) }\end{array}$ & $\begin{array}{l}\text { Sample time } \\
\text { (hh:mm) }\end{array}$ & $\begin{array}{c}\text { Fenhexamid } \\
\text { [67622] }\end{array}$ & $\begin{array}{c}\text { Fipronil } \\
\text { sulfide } \\
\text { [66610] }\end{array}$ & $\begin{array}{l}\text { Fipronil } \\
\text { sulfone } \\
\text { [66613] }\end{array}$ & $\begin{array}{c}\text { Fipronil } \\
\text { [66604] }\end{array}$ & $\begin{array}{c}\text { Fluridone } \\
\text { [51864] }\end{array}$ & $\begin{array}{c}\text { Flusilazole } \\
\text { [67649] }\end{array}$ & $\begin{array}{c}\text { Fluxapyroxad } \\
\text { [51851] }\end{array}$ & $\begin{array}{l}\text { Hexazinone } \\
\text { [65085] }\end{array}$ & $\begin{array}{c}\text { Imazalil } \\
\text { [67662] }\end{array}$ & $\begin{array}{c}\text { Imidacloprid } \\
\text { [68426] }\end{array}$ & $\begin{array}{c}\text { Indoxacarb } \\
\text { [68627] }\end{array}$ & $\begin{array}{c}\text { Iprodione } \\
\text { [66617] }\end{array}$ \\
\hline \multicolumn{14}{|c|}{ Mokelumne River at New Hope Road-Continued } \\
\hline $04 / 19 / 2016$ & 09:20 & - & - & - & - & - & - & 6.8 & 17.7 & - & - & - & - \\
\hline $05 / 18 / 2016$ & 09:30 & - & - & - & - & - & - & - & 9.9 & - & - & - & - \\
\hline $06 / 15 / 2016$ & $09: 15$ & - & - & - & - & - & - & - & 11.8 & - & - & - & - \\
\hline \multicolumn{14}{|c|}{ Sacramento River at Hood-Continued } \\
\hline $07 / 28 / 2015$ & $08: 45$ & - & - & - & 25 & - & - & - & 43.8 & - & - & - & - \\
\hline 08/18/2015 & $08: 40$ & - & - & - & 6.9 & 4.5 & - & E2.0 & 22.9 & - & E2.3 & - & - \\
\hline $09 / 23 / 2015$ & 08:30 & 8.5 & - & - & - & - & - & - & 11.6 & 100 & - & - & - \\
\hline $10 / 21 / 2015$ & 08:00 & - & - & - & 4.4 & - & - & - & 20 & - & - & - & - \\
\hline $11 / 10 / 2015$ & 09:00 & - & - & - & 12.2 & - & - & - & 27.2 & - & E1.9 & - & - \\
\hline $12 / 15 / 2015$ & $15: 00$ & - & - & - & - & - & - & - & E8.2 & - & - & - & - \\
\hline *01/19/2016 & $08: 30$ & - & - & - & - & - & 7.5 & - & 9 & - & - & - & - \\
\hline $02 / 17 / 2016$ & 08:00 & 28.1 & - & - & - & - & - & - & E7.0 & - & E2.2 & - & - \\
\hline *03/07/2016 & $14: 20$ & - & - & - & - & - & - & - & - & - & 9.3 & - & 6 \\
\hline 04/19/2016 & $08: 20$ & - & - & - & 4.6 & - & - & - & 19.4 & - & - & - & - \\
\hline 05/18/2016 & 08:30 & - & - & - & - & - & - & - & 14.8 & - & - & - & - \\
\hline $06 / 15 / 2016$ & 08:10 & - & - & - & 3.1 & - & - & - & 17.5 & - & - & - & - \\
\hline \multicolumn{14}{|c|}{ San Joaquin River at Buckley Cove-Continued } \\
\hline $07 / 28 / 2015$ & $11: 10$ & - & 10.8 & 12.2 & 13.5 & 383 & - & 15.4 & 132 & - & E3.2 & - & - \\
\hline $08 / 18 / 2015$ & $11: 10$ & - & 4.6 & 3.9 & - & 293 & - & 32.4 & 94.8 & - & 5.2 & - & - \\
\hline $09 / 23 / 2015$ & $10: 45$ & - & 3 & - & - & 165 & - & 22.6 & 39 & - & 9.7 & - & - \\
\hline $10 / 21 / 2015$ & $10: 50$ & - & - & - & - & 57 & - & 35.8 & 33 & - & - & - & - \\
\hline $11 / 10 / 2015$ & $11: 30$ & - & - & - & E1.8 & 9.6 & - & 21.7 & 19.2 & - & - & - & - \\
\hline $12 / 15 / 2015$ & $12: 20$ & - & - & - & - & 23.4 & - & - & E8.3 & - & 4.6 & - & - \\
\hline *01/19/2016 & $10: 50$ & - & - & - & 3.4 & 11.3 & - & 10 & 30.7 & - & 17.8 & - & 34.6 \\
\hline $02 / 17 / 2016$ & $10: 10$ & - & - & - & - & 16.3 & - & 11.4 & 13.5 & - & 8.9 & - & 51.1 \\
\hline *03/07/2016 & $10: 15$ & - & - & - & - & 25.6 & - & $\mathrm{E} 4.7$ & 16.6 & - & 7.2 & - & 42.1 \\
\hline 04/19/2016 & $10: 40$ & - & - & - & 4.4 & 230 & - & 57.7 & 54.5 & - & 60.1 & - & - \\
\hline 05/18/2016 & $10: 45$ & - & - & - & - & 208 & - & 7.2 & 10.3 & - & 13.7 & - & - \\
\hline $06 / 15 / 2016$ & $10: 50$ & - & - & - & - & 213 & - & 11.1 & 21.2 & - & 7.3 & - & - \\
\hline
\end{tabular}


Table 7. Pesticide concentrations with measured detections in environmental water samples collected in the Sacramento-San Joaquin Delta, 2015-16.-Continued

[Numbers in brackets are U.S. Geological Survey (USGS) National Water Information System (NWIS) parameter codes. Concentrations are in nanograms per liter. Abbreviations: E, estimated; hh:mm, hour:minute; mm/dd/yyyy, month/day/year; —, not detected; *, storm sample]

\begin{tabular}{|c|c|c|c|c|c|c|c|c|c|c|c|c|c|}
\hline $\begin{array}{l}\text { Sample date } \\
\text { (mm/dd/yyyy) }\end{array}$ & $\begin{array}{l}\text { Sample time } \\
\text { (hh:mm) }\end{array}$ & $\begin{array}{c}\text { Fenhexamid } \\
\text { [67622] }\end{array}$ & $\begin{array}{c}\text { Fipronil } \\
\text { sulfide } \\
\text { [66610] }\end{array}$ & $\begin{array}{l}\text { Fipronil } \\
\text { sulfone } \\
\text { [66613] }\end{array}$ & $\begin{array}{l}\text { Fipronil } \\
\text { [66604] }\end{array}$ & $\begin{array}{c}\text { Fluridone } \\
\text { [51864] }\end{array}$ & $\begin{array}{c}\text { Flusilazole } \\
\text { [67649] }\end{array}$ & $\begin{array}{c}\text { Fluxapyroxad } \\
\text { [51851] }\end{array}$ & $\begin{array}{c}\text { Hexazinone } \\
\text { [65085] }\end{array}$ & $\begin{array}{c}\text { Imazalil } \\
\text { [67662] }\end{array}$ & $\begin{array}{c}\text { Imidacloprid } \\
\text { [68426] }\end{array}$ & $\begin{array}{c}\text { Indoxacarb } \\
\text { [68627] }\end{array}$ & $\begin{array}{c}\text { Iprodione } \\
\text { [66617] }\end{array}$ \\
\hline \multicolumn{14}{|c|}{ San Joaquin River near Vernalis-Continued } \\
\hline $07 / 28 / 2015$ & $12: 15$ & - & - & - & - & 4.4 & - & - & 38.8 & - & - & - & - \\
\hline 08/18/2015 & $12: 50$ & - & - & - & - & - & - & 5.8 & 22.9 & - & - & - & - \\
\hline $09 / 23 / 2015$ & $12: 20$ & - & - & - & - & - & - & - & 11.4 & - & - & - & - \\
\hline $10 / 21 / 2015$ & $12: 40$ & - & - & - & - & - & - & $\mathrm{E} 4.0$ & 12.9 & - & - & - & - \\
\hline $11 / 10 / 2015$ & $13: 00$ & - & - & - & E2.4 & - & - & 6.6 & 10.9 & - & - & - & - \\
\hline $12 / 15 / 2015$ & $11: 00$ & 12.1 & - & - & - & - & - & - & 8.9 & - & E2.9 & - & - \\
\hline *01/19/2016 & $12: 30$ & - & - & - & $\mathrm{E} 2.1$ & - & 12.7 & - & 46.4 & - & 12.4 & - & 124 \\
\hline $02 / 17 / 2016$ & $12: 00$ & - & - & - & - & E2.4 & - & 6.3 & 9.5 & - & 4.3 & - & 201 \\
\hline *03/07/2016 & $11: 50$ & - & - & - & - & $\mathrm{E} 2.7$ & - & $\mathrm{E} 4.3$ & 17.9 & - & 8.3 & - & 54.2 \\
\hline 04/19/2016 & $12: 45$ & - & - & - & - & $\mathrm{E} 2.5$ & - & 24.7 & 33.5 & - & 11.1 & - & - \\
\hline $05 / 18 / 2016$ & $12: 20$ & - & - & - & - & - & - & 6.9 & 8.7 & - & - & - & - \\
\hline $06 / 15 / 2016$ & $12: 40$ & - & - & - & - & - & - & 7.9 & 11.7 & - & - & - & - \\
\hline \multicolumn{14}{|c|}{ Ulatis Creek at Browns Road-Continued } \\
\hline $07 / 28 / 2015$ & $14: 20$ & - & - & - & - & 11.6 & - & 14.8 & 67.8 & - & 12.6 & - & - \\
\hline $08 / 18 / 2015$ & $15: 00$ & - & - & - & - & 6.7 & - & 32.6 & 72.2 & - & 4.6 & - & - \\
\hline $09 / 23 / 2015$ & $14: 15$ & - & - & - & - & 4.5 & - & $\mathrm{E} 2.7$ & - & - & 3.9 & - & - \\
\hline $10 / 21 / 2015$ & $15: 00$ & - & - & - & 13.4 & 5.4 & - & 13.8 & 32.9 & - & 30 & - & - \\
\hline $11 / 10 / 2015$ & $15: 30$ & - & - & 4.7 & 3.8 & E2.1 & - & 13.3 & 41.4 & - & 16 & - & - \\
\hline $12 / 15 / 2015$ & 08:50 & 73.1 & - & 4.6 & 4.1 & - & - & - & 14.1 & - & 21.9 & - & - \\
\hline *01/19/2016 & $15: 00$ & - & - & - & 11 & - & - & - & 32.2 & - & 19.5 & - & - \\
\hline 02/17/2016 & $14: 10$ & - & - & - & - & $\mathrm{E} 2.0$ & - & - & 13.2 & - & 17.8 & - & - \\
\hline *03/07/2016 & $08: 30$ & - & - & 4.8 & 4 & 23.3 & - & 71.4 & 2,270 & - & 19.5 & 72.9 & - \\
\hline 04/19/2016 & $15: 10$ & - & - & - & - & 95.8 & - & 15.2 & 78.2 & - & 9.6 & - & - \\
\hline $05 / 18 / 2016$ & $14: 20$ & - & - & - & - & 12.7 & - & 7.6 & 112 & - & 7.8 & - & - \\
\hline $06 / 15 / 2016$ & $14: 40$ & - & - & - & - & 3.8 & - & - & 86.6 & - & 6.4 & - & - \\
\hline
\end{tabular}


Table 7. Pesticide concentrations with measured detections in environmental water samples collected in the Sacramento-San Joaquin Delta, 2015-16.-Continued

[Numbers in brackets are U.S. Geological Survey (USGS) National Water Information System (NWIS) parameter codes. Concentrations are in nanograms per liter. Abbreviations: E, estimated; hh:mm, hour:minute; mm/dd/yyyy, month/day/year; —, not detected; *, storm sample]

\begin{tabular}{|c|c|c|c|c|c|c|c|c|c|c|c|c|}
\hline $\begin{array}{l}\text { Sample date } \\
\text { (mm/dd/yyyy) }\end{array}$ & $\begin{array}{c}\text { Sample } \\
\text { time } \\
\text { (hh:mm) }\end{array}$ & $\begin{array}{c}\text { Metalaxyl } \\
\text { [68437] }\end{array}$ & $\begin{array}{c}\text { Methoxyfenozide } \\
\text { [68647] }\end{array}$ & $\begin{array}{c}\text { Metolachlor } \\
\text { [65090] }\end{array}$ & $\begin{array}{c}\text { Myclobutanil } \\
\text { [66632] }\end{array}$ & $\begin{array}{l}\text { Oryzalin } \\
\text { [68663] }\end{array}$ & $\begin{array}{c}\text { Oxadiazon } \\
\text { [51843] }\end{array}$ & $\begin{array}{c}\text { Oxyfluorfen } \\
\text { [65093] }\end{array}$ & $\begin{array}{c}\text { Pendimethalin } \\
\text { [65098] }\end{array}$ & $\begin{array}{c}\text { Penoxsulam } \\
\text { [51863] }\end{array}$ & $\begin{array}{c}\text { Piperonyl } \\
\text { butoxide } \\
\text { [65102] }\end{array}$ & $\begin{array}{c}\text { Prodiamine } \\
\text { [51844] }\end{array}$ \\
\hline \multicolumn{13}{|c|}{ Mokelumne River at New Hope Road-Continued } \\
\hline $07 / 28 / 2015$ & 09:50 & - & 3.2 & 14.2 & - & - & - & - & - & - & - & - \\
\hline $08 / 18 / 2015$ & 09:50 & - & 11.8 & 8 & 15.2 & - & - & - & - & - & 3.9 & - \\
\hline $09 / 23 / 2015$ & 09:20 & - & - & 4.4 & - & - & - & - & - & - & - & - \\
\hline $10 / 21 / 2015$ & $09: 10$ & - & - & - & - & - & - & - & - & - & - & - \\
\hline $11 / 10 / 2015$ & 10:00 & - & - & - & - & - & - & - & - & - & 14.6 & - \\
\hline $12 / 15 / 2015$ & $14: 00$ & - & - & - & - & - & - & 7.7 & - & - & 4.1 & - \\
\hline *01/19/2016 & $09: 45$ & - & - & - & - & - & - & 16.7 & 17.7 & - & 6.9 & - \\
\hline $02 / 17 / 2016$ & 09:00 & - & - & - & - & - & - & - & - & - & - & - \\
\hline *03/07/2016 & $13: 30$ & - & 6.4 & - & - & E2.6 & - & 6.3 & - & - & - & - \\
\hline 04/19/2016 & 09:20 & - & - & - & - & - & - & - & - & - & - & - \\
\hline 05/18/2016 & $09: 30$ & - & - & - & - & - & - & - & - & - & - & - \\
\hline $06 / 15 / 2016$ & $09: 15$ & - & - & - & - & - & - & - & - & - & - & - \\
\hline \multicolumn{13}{|c|}{ Sacramento River at Hood-Continued } \\
\hline $07 / 28 / 2015$ & $08: 45$ & - & - & 13.2 & - & - & - & - & - & - & 36.3 & - \\
\hline $08 / 18 / 2015$ & $08: 40$ & - & - & 3.9 & - & - & - & - & - & - & 17.8 & - \\
\hline $09 / 23 / 2015$ & $08: 30$ & - & - & - & - & - & - & - & - & - & 10.2 & - \\
\hline $10 / 21 / 2015$ & 08:00 & - & - & - & - & - & - & - & - & - & 4.7 & - \\
\hline $11 / 10 / 2015$ & 09:00 & - & - & - & - & - & - & - & - & - & 45 & - \\
\hline $12 / 15 / 2015$ & $15: 00$ & - & - & - & - & - & - & 3.8 & - & - & 7.4 & - \\
\hline$* 01 / 19 / 2016$ & $08: 30$ & - & 3.2 & - & - & - & - & - & - & - & - & - \\
\hline $02 / 17 / 2016$ & 08:00 & - & - & - & - & - & - & - & - & - & - & - \\
\hline *03/07/2016 & $14: 20$ & - & - & 13.6 & - & 25 & - & - & - & - & - & - \\
\hline $04 / 19 / 2016$ & $08: 20$ & - & - & - & - & - & - & - & - & - & 10.4 & - \\
\hline 05/18/2016 & $08: 30$ & - & - & 5.9 & - & - & - & - & - & - & - & - \\
\hline $06 / 15 / 2016$ & 08:10 & - & - & 7.1 & - & - & - & - & - & - & 4.4 & - \\
\hline \multicolumn{13}{|c|}{ San Joaquin River at Buckley Cove-Continued } \\
\hline $07 / 28 / 2015$ & $11: 10$ & - & 47 & 143 & - & - & - & - & - & - & - & - \\
\hline $08 / 18 / 2015$ & $11: 10$ & - & 48.8 & 94.5 & - & - & - & - & - & - & - & - \\
\hline $09 / 23 / 2015$ & $10: 45$ & - & 63.9 & 43.3 & - & - & - & - & - & - & 6.1 & - \\
\hline
\end{tabular}


Table 7. Pesticide concentrations with measured detections in environmental water samples collected in the Sacramento-San Joaquin Delta, 2015-16.-Continued

[Numbers in brackets are U.S. Geological Survey (USGS) National Water Information System (NWIS) parameter codes. Concentrations are in nanograms per liter. Abbreviations: E, estimated; hh:mm, hour:minute; mm/dd/yyyy, month/day/year; —, not detected; *, storm sample]

\begin{tabular}{|c|c|c|c|c|c|c|c|c|c|c|c|c|}
\hline $\begin{array}{l}\text { Sample date } \\
\text { (mm/dd/yyyy) }\end{array}$ & $\begin{array}{c}\text { Sample } \\
\text { time } \\
\text { (hh:mm) }\end{array}$ & $\begin{array}{c}\text { Metalaxyl } \\
\text { [68437] }\end{array}$ & $\begin{array}{c}\text { Methoxyfenozide } \\
\text { [68647] }\end{array}$ & $\begin{array}{l}\text { Metolachlor } \\
\text { [65090] }\end{array}$ & $\begin{array}{l}\text { Myclobutanil } \\
\text { [66632] }\end{array}$ & $\begin{array}{l}\text { Oryzalin } \\
\text { [68663] }\end{array}$ & $\begin{array}{l}\text { Oxadiazon } \\
\text { [51843] }\end{array}$ & $\begin{array}{c}\text { Oxyfluorfen } \\
\text { [65093] }\end{array}$ & $\begin{array}{l}\text { Pendimethalin } \\
\text { [65098] }\end{array}$ & $\begin{array}{c}\text { Penoxsulam } \\
{[51863]}\end{array}$ & $\begin{array}{c}\text { Piperonyl } \\
\text { butoxide } \\
\text { [65102] }\end{array}$ & $\begin{array}{c}\text { Prodiamine } \\
\text { [51844] }\end{array}$ \\
\hline \multicolumn{13}{|c|}{ San Joaquin River at Buckley Cove-Continued } \\
\hline $10 / 21 / 2015$ & $10: 50$ & - & 55.8 & 16.5 & - & - & - & - & - & - & 8.3 & - \\
\hline $11 / 10 / 2015$ & $11: 30$ & - & 8.1 & 12.2 & - & - & - & - & - & - & 20.5 & - \\
\hline $12 / 15 / 2015$ & $12: 20$ & - & 15.7 & 7 & - & - & - & - & - & - & 16.8 & - \\
\hline$* 01 / 19 / 2016$ & $10: 50$ & 36.9 & 254 & 16.2 & 15.9 & 17.5 & 7.7 & 68.2 & 53.8 & - & 32.1 & - \\
\hline $02 / 17 / 2016$ & 10:10 & - & 85.4 & 10.8 & - & $\mathrm{E} 4.7$ & 9.8 & - & - & - & 6 & - \\
\hline *03/07/2016 & $10: 15$ & - & 45.2 & 42.8 & - & 16.7 & 11.5 & 211 & 33.4 & - & - & - \\
\hline $04 / 19 / 2016$ & $10: 40$ & - & 74.3 & 55.6 & - & 15.1 & - & - & - & - & 7.2 & - \\
\hline $05 / 18 / 2016$ & $10: 45$ & - & 11.5 & 42.6 & - & - & - & - & - & - & - & - \\
\hline $06 / 15 / 2016$ & $10: 50$ & - & 21.7 & 83.5 & - & - & - & - & 14.4 & - & - & - \\
\hline \multicolumn{13}{|c|}{ San Joaquin River near Vernalis-Continued } \\
\hline $07 / 28 / 2015$ & $12: 15$ & - & 18.9 & 30.8 & - & - & - & - & - & - & - & - \\
\hline $08 / 18 / 2015$ & $12: 50$ & - & 10.5 & 5.9 & - & - & - & - & - & - & - & - \\
\hline $09 / 23 / 2015$ & $12: 20$ & - & 16.6 & - & - & - & - & - & - & - & - & - \\
\hline $10 / 21 / 2015$ & $12: 40$ & - & 13.8 & 3.5 & - & - & - & - & - & - & - & - \\
\hline $11 / 10 / 2015$ & 13:00 & - & 5.2 & 6.4 & - & - & - & - & - & - & - & - \\
\hline $12 / 15 / 2015$ & $11: 00$ & - & 8.5 & - & - & - & - & - & - & - & - & - \\
\hline *01/19/2016 & $12: 30$ & - & 67.2 & 28.3 & - & 8.7 & - & 22.6 & 46 & - & - & - \\
\hline $02 / 17 / 2016$ & $12: 00$ & - & 28.2 & - & - & - & - & - & - & - & - & - \\
\hline *03/07/2016 & $11: 50$ & - & 36 & 12.6 & - & 10.1 & - & - & 28.9 & - & - & - \\
\hline $04 / 19 / 2016$ & $12: 45$ & - & 14 & 9 & - & - & - & - & - & - & - & - \\
\hline 05/18/2016 & $12: 20$ & - & 7.6 & 11.8 & - & - & - & - & - & - & - & - \\
\hline $06 / 15 / 2016$ & $12: 40$ & - & 22.8 & 14.8 & - & - & - & - & - & - & - & - \\
\hline \multicolumn{13}{|c|}{ Ulatis Creek at Browns Road-Continued } \\
\hline $07 / 28 / 2015$ & $14: 20$ & - & 3.8 & 140 & - & - & - & - & - & - & - & - \\
\hline $08 / 18 / 2015$ & $15: 00$ & - & 5.3 & 63 & - & - & - & - & - & - & - & - \\
\hline $09 / 23 / 2015$ & $14: 15$ & 17 & - & - & - & - & - & - & - & - & - & - \\
\hline $10 / 21 / 2015$ & $15: 00$ & - & 9 & 14.5 & - & - & - & - & - & - & - & - \\
\hline $11 / 10 / 2015$ & $15: 30$ & - & - & 20.9 & - & - & - & - & - & - & - & - \\
\hline $12 / 15 / 2015$ & $08: 50$ & - & - & - & - & - & - & 29.7 & - & E3.1 & - & - \\
\hline
\end{tabular}


Table 7. Pesticide concentrations with measured detections in environmental water samples collected in the Sacramento-San Joaquin Delta, 2015-16.-Continued

[Numbers in brackets are U.S. Geological Survey (USGS) National Water Information System (NWIS) parameter codes. Concentrations are in nanograms per liter. Abbreviations: E, estimated; hh:mm, hour:minute; mm/dd/yyyy, month/day/year; —, not detected; *, storm sample]

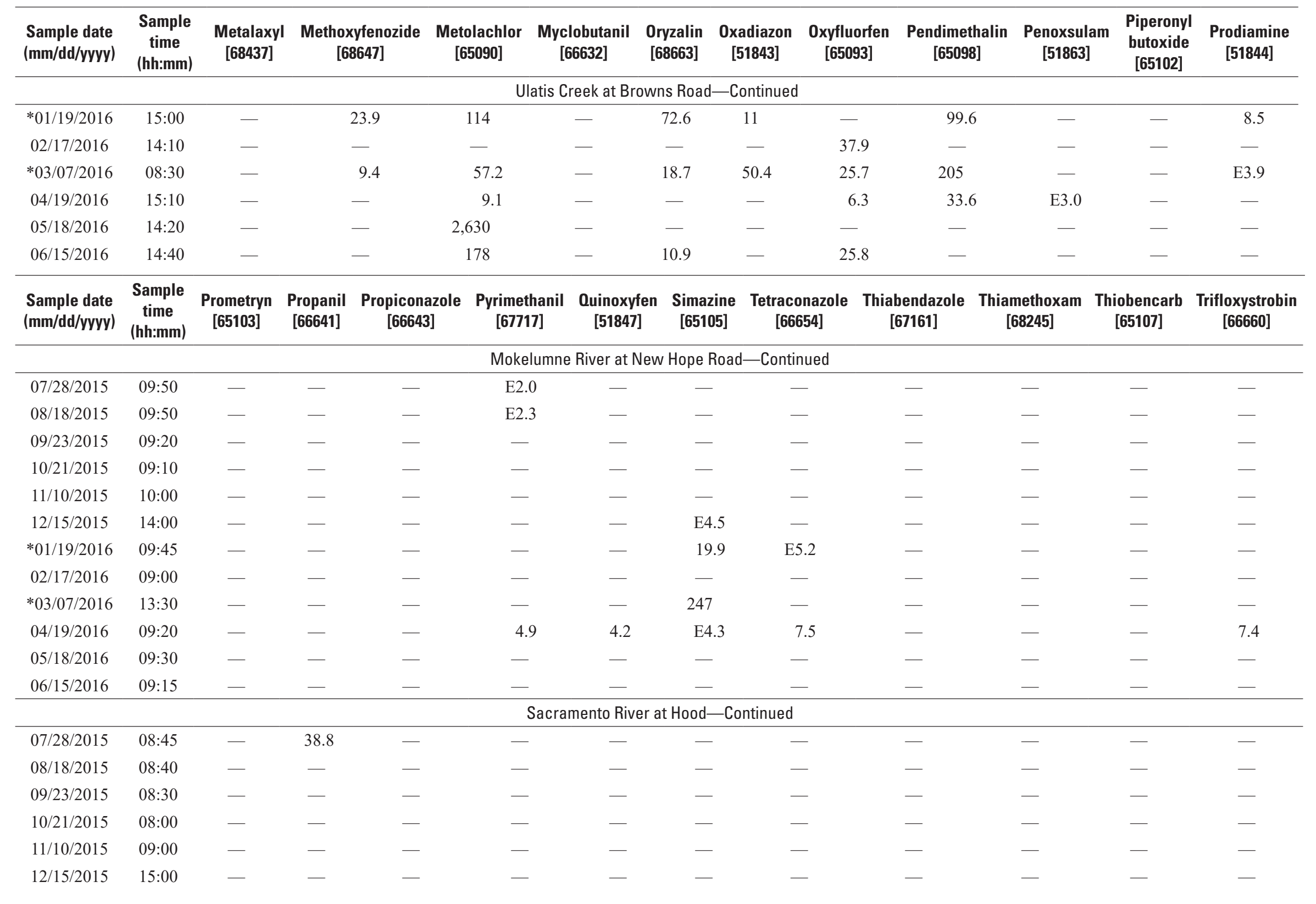


Table 7. Pesticide concentrations with measured detections in environmental water samples collected in the Sacramento-San Joaquin Delta, 2015-16.-Continued

[Numbers in brackets are U.S. Geological Survey (USGS) National Water Information System (NWIS) parameter codes. Concentrations are in nanograms per liter. Abbreviations: E, estimated; hh:mm, hour:minute; mm/dd/yyyy, month/day/year; —, not detected; *, storm sample]

\begin{tabular}{|c|c|c|c|c|c|c|c|c|c|c|c|c|}
\hline $\begin{array}{l}\text { Sample date } \\
\text { (mm/dd/yyyy) }\end{array}$ & $\begin{array}{c}\text { Sample } \\
\text { time } \\
\text { (hh:mm) }\end{array}$ & $\begin{array}{c}\text { Prometryn } \\
\text { [65103] }\end{array}$ & $\begin{array}{c}\text { Propanil } \\
\text { [66641] }\end{array}$ & $\begin{array}{c}\text { Propiconazole } \\
\text { [66643] }\end{array}$ & $\begin{array}{c}\text { Pyrimethanil } \\
\text { [67717] }\end{array}$ & $\begin{array}{c}\text { Quinoxyfen } \\
\text { [51847] }\end{array}$ & $\begin{array}{c}\text { Simazine } \\
\text { [65105] }\end{array}$ & $\begin{array}{c}\text { Tetraconazole } \\
\text { [66654] }\end{array}$ & $\begin{array}{c}\text { Thiabendazole } \\
\text { [67161] }\end{array}$ & $\begin{array}{c}\text { Thiamethoxam } \\
\text { [68245] }\end{array}$ & $\begin{array}{c}\text { Thiobencarb } \\
\text { [65107] }\end{array}$ & $\begin{array}{c}\text { Trifloxystrobin } \\
\text { [66660] }\end{array}$ \\
\hline \multicolumn{13}{|c|}{ Sacramento River at Hood_-Continued } \\
\hline$* 01 / 19 / 2016$ & 08:30 & - & - & - & - & - & 5.9 & - & - & - & - & - \\
\hline $02 / 17 / 2016$ & 08:00 & - & - & - & - & - & - & - & - & - & - & - \\
\hline$* 03 / 07 / 2016$ & $14: 20$ & - & - & E3.5 & - & - & 23.9 & - & - & - & - & - \\
\hline $04 / 19 / 2016$ & $08: 20$ & - & - & - & - & - & E3.5 & - & - & - & - & - \\
\hline 05/18/2016 & $08: 30$ & - & - & - & - & - & - & - & - & - & 21.5 & - \\
\hline $06 / 15 / 2016$ & $08: 10$ & - & 27.6 & - & - & - & - & - & - & - & - & - \\
\hline \multicolumn{13}{|c|}{ San Joaquin River at Buckley Cove-Continued } \\
\hline 07/28/2015 & $11: 10$ & - & - & - & E2.6 & - & 53.5 & - & - & - & - & - \\
\hline 08/18/2015 & $11: 10$ & - & - & - & E3.0 & - & 26 & - & E3.1 & - & - & - \\
\hline $09 / 23 / 2015$ & $10: 45$ & - & - & - & - & - & 6.9 & - & - & 3.4 & - & - \\
\hline $10 / 21 / 2015$ & $10: 50$ & - & - & - & - & - & 6.5 & - & - & - & - & - \\
\hline $11 / 10 / 2015$ & $11: 30$ & - & - & - & - & - & E4.2 & - & - & - & - & - \\
\hline $12 / 15 / 2015$ & $12: 20$ & - & - & - & - & - & E4.9 & - & E3.0 & - & - & - \\
\hline$* 01 / 19 / 2016$ & $10: 50$ & - & - & - & - & - & 387 & - & - & - & - & - \\
\hline $02 / 17 / 2016$ & 10:10 & - & - & - & - & - & 131 & - & - & - & - & - \\
\hline$* 03 / 07 / 2016$ & $10: 15$ & - & - & - & E2.0 & - & 192 & - & - & - & - & - \\
\hline 04/19/2016 & $10: 40$ & - & - & 38.7 & - & - & 39.4 & - & 7.0 & - & - & - \\
\hline 05/18/2016 & $10: 45$ & - & - & - & - & - & 5.9 & - & 5.5 & - & - & - \\
\hline $06 / 15 / 2016$ & $10: 50$ & - & - & - & - & - & 16.5 & - & - & - & - & - \\
\hline \multicolumn{13}{|c|}{ San Joaquin River near Vernalis_-Continued } \\
\hline $07 / 28 / 2015$ & $12: 15$ & - & - & - & - & - & 17 & - & - & - & - & - \\
\hline $08 / 18 / 2015$ & $12: 50$ & - & - & - & - & - & 5.3 & - & - & - & - & - \\
\hline $09 / 23 / 2015$ & $12: 20$ & - & - & - & - & - & E3.3 & - & - & - & - & - \\
\hline $10 / 21 / 2015$ & $12: 40$ & - & - & - & - & - & - & - & - & - & - & - \\
\hline $11 / 10 / 2015$ & 13:00 & - & - & 23.5 & - & - & 7.3 & - & - & - & - & - \\
\hline $12 / 15 / 2015$ & $11: 00$ & - & - & - & - & - & 8.4 & - & - & - & - & - \\
\hline *01/19/2016 & $12: 30$ & - & - & 23.4 & - & - & 30.6 & 11.6 & - & - & - & - \\
\hline $02 / 17 / 2016$ & $12: 00$ & - & - & 16.4 & - & - & 23.8 & - & - & - & - & - \\
\hline$* 03 / 07 / 2016$ & $11: 50$ & - & - & E4.8 & - & - & 21.6 & - & - & - & - & - \\
\hline
\end{tabular}


Table 7. Pesticide concentrations with measured detections in environmental water samples collected in the Sacramento-San Joaquin Delta, 2015-16.-Continued

[Numbers in brackets are U.S. Geological Survey (USGS) National Water Information System (NWIS) parameter codes. Concentrations are in nanograms per liter. Abbreviations: E, estimated; hh:mm, hour:minute; mm/dd/yyyy, month/day/year; —, not detected; *, storm sample]

\begin{tabular}{|c|c|c|c|c|c|c|c|c|c|c|c|c|}
\hline $\begin{array}{l}\text { Sample date } \\
\text { (mm/dd/yyyy) }\end{array}$ & $\begin{array}{c}\text { Sample } \\
\text { time } \\
\text { (hh:mm) }\end{array}$ & $\begin{array}{c}\text { Prometryn } \\
\text { [65103] }\end{array}$ & $\begin{array}{c}\text { Propanil } \\
\text { [66641] }\end{array}$ & $\begin{array}{c}\text { Propiconazole } \\
\text { [66643] }\end{array}$ & $\begin{array}{c}\text { Pyrimethanil } \\
\text { [67717] }\end{array}$ & $\begin{array}{c}\text { Quinoxyfen } \\
\text { [51847] }\end{array}$ & $\begin{array}{c}\text { Simazine } \\
\text { [65105] }\end{array}$ & $\begin{array}{c}\text { Tetraconazole } \\
{[66654]}\end{array}$ & $\begin{array}{c}\text { Thiabendazole } \\
\text { [67161] }\end{array}$ & $\begin{array}{c}\text { Thiamethoxam } \\
\text { [68245] }\end{array}$ & $\begin{array}{c}\text { Thiobencarb } \\
\text { [65107] }\end{array}$ & $\begin{array}{c}\text { Trifloxystrobin } \\
{[66660]}\end{array}$ \\
\hline \multicolumn{13}{|c|}{ San Joaquin River near Vernalis_-Continued } \\
\hline $04 / 19 / 2016$ & $12: 45$ & 10.2 & - & 23.5 & - & - & 10.2 & - & - & - & - & - \\
\hline 05/18/2016 & $12: 20$ & - & - & - & - & - & - & - & - & - & - & - \\
\hline $06 / 15 / 2016$ & $12: 40$ & - & - & - & - & - & - & - & - & - & - & - \\
\hline \multicolumn{13}{|c|}{ Ulatis Creek at Browns Road-Continued } \\
\hline $07 / 28 / 2015$ & $14: 20$ & - & - & - & - & - & 54.5 & - & - & - & - & - \\
\hline 08/18/2015 & $15: 00$ & - & - & - & E2.4 & - & 29.5 & - & E2.7 & - & - & - \\
\hline 09/23/2015 & $14: 15$ & - & - & - & - & - & 13.5 & - & - & - & - & - \\
\hline $10 / 21 / 2015$ & $15: 00$ & - & - & - & - & - & - & - & - & - & - & - \\
\hline 11/10/2015 & $15: 30$ & - & - & - & - & - & 9.5 & - & - & - & - & - \\
\hline $12 / 15 / 2015$ & 08:50 & - & - & - & - & - & - & - & 3.9 & - & - & - \\
\hline *01/19/2016 & $15: 00$ & - & - & 34 & - & - & 6.9 & 10.3 & - & - & - & - \\
\hline $02 / 17 / 2016$ & $14: 10$ & - & - & - & - & - & 15.3 & - & 4 & - & - & - \\
\hline *03/07/2016 & $08: 30$ & - & - & 24.8 & - & - & 209 & - & - & - & - & 7.7 \\
\hline $04 / 19 / 2016$ & $15: 10$ & - & - & 35.1 & - & - & 12.6 & - & $\mathrm{E} 2.3$ & - & - & - \\
\hline 05/18/2016 & $14: 20$ & - & - & - & - & - & 159 & - & - & 25.7 & - & - \\
\hline $06 / 15 / 2016$ & $14: 40$ & - & - & - & - & - & 16.2 & - & - & - & - & - \\
\hline
\end{tabular}


Table 8. Pesticide concentrations with measured detections in suspended sediments filtered from environmental water samples collected in the Sacramento-San Joaquin Delta, 2015-16.

[Numbers in brackets are U.S. Geological Survey (USGS) National Water Information System (NWIS) parameter codes. Concentrations are in nanogram per liter. Abbreviations: E, estimated; hh:mm, hour:minute; mm/dd/yyyy, month/day/year; —, not detected; *, storm sample]

\begin{tabular}{|c|c|c|c|c|c|c|c|c|c|c|c|c|}
\hline $\begin{array}{l}\text { Sample date } \\
\text { (mm/dd/yyyy) }\end{array}$ & $\begin{array}{c}\text { Sample time } \\
\text { (hh:mm) }\end{array}$ & $\begin{array}{l}\text { Bifenthrin } \\
\text { [65067] }\end{array}$ & $\begin{array}{c}\text { Cyhalothrin } \\
\text { (all isomers) } \\
\text { [68354] }\end{array}$ & $\begin{array}{c}\text { DCPA } \\
{[65076]}\end{array}$ & $\begin{array}{c}\text { Dithiopyr } \\
\text { [51837] }\end{array}$ & $\begin{array}{c}\text { Metalaxyl } \\
\text { [68437] }\end{array}$ & $\begin{array}{c}\text { Metolachlor } \\
\text { [65090] }\end{array}$ & $\begin{array}{l}\text { Oxyfluorfen } \\
\text { [65093] }\end{array}$ & $\begin{array}{c}\text { p,p'-DDE } \\
\text { [65095] }\end{array}$ & $\begin{array}{l}\text { Pendimethalin } \\
\text { [65098] }\end{array}$ & $\begin{array}{l}\text { Permethrin } \\
\text { [65099] }\end{array}$ & $\begin{array}{c}\text { Prodiamine } \\
\text { [51844] }\end{array}$ \\
\hline \multicolumn{13}{|c|}{ Mokelumne River at New Hope Road } \\
\hline $07 / 28 / 2015$ & 09:50 & - & - & - & - & - & - & - & - & - & - & - \\
\hline $08 / 18 / 2015$ & 09:50 & - & - & - & - & - & - & - & - & - & - & - \\
\hline $09 / 23 / 2015$ & 09:20 & - & - & - & - & - & - & - & - & - & - & - \\
\hline $10 / 21 / 2015$ & 09:10 & - & - & - & - & - & - & - & - & - & - & - \\
\hline $11 / 10 / 2015$ & $10: 00$ & - & - & - & - & - & - & - & - & - & - & - \\
\hline $12 / 15 / 2015$ & $14: 00$ & - & - & - & - & - & - & - & - & - & - & - \\
\hline *01/19/2016 & $09: 45$ & - & - & - & - & - & - & - & - & - & - & - \\
\hline $02 / 17 / 2016$ & 09:00 & - & - & - & - & - & - & - & - & - & - & - \\
\hline$* 03 / 07 / 2016$ & $13: 30$ & - & - & - & - & - & - & - & - & - & - & - \\
\hline $04 / 19 / 2016$ & 09:20 & - & - & - & - & - & - & - & - & - & - & - \\
\hline $05 / 18 / 2016$ & 09:30 & - & - & - & - & - & - & - & - & - & - & - \\
\hline $06 / 15 / 2016$ & $09: 15$ & - & - & - & - & - & - & - & - & - & - & - \\
\hline \multicolumn{13}{|c|}{ Sacramento River at Hood } \\
\hline $07 / 28 / 2015$ & $08: 45$ & - & - & - & - & - & - & - & - & - & - & - \\
\hline $08 / 18 / 2015$ & $08: 40$ & - & - & - & - & - & - & - & - & - & - & - \\
\hline $09 / 23 / 2015$ & $08: 30$ & - & - & - & - & - & - & - & - & - & - & - \\
\hline $10 / 21 / 2015$ & 08:00 & - & - & - & - & - & - & - & - & - & - & - \\
\hline $11 / 10 / 2015$ & 09:00 & - & - & - & - & - & - & - & - & - & - & - \\
\hline $12 / 15 / 2015$ & $15: 00$ & - & - & - & - & - & - & - & - & - & - & - \\
\hline$* 01 / 19 / 2016$ & 08:30 & - & - & - & - & - & - & - & - & - & - & - \\
\hline 02/17/2016 & 08:00 & - & - & - & - & - & - & - & - & - & - & - \\
\hline$* 03 / 07 / 2016$ & $14: 20$ & - & - & - & - & - & - & - & - & - & 19.2 & - \\
\hline $04 / 19 / 2016$ & 08:20 & - & - & - & - & - & - & - & - & - & - & - \\
\hline $05 / 18 / 2016$ & 08:30 & - & - & - & - & - & - & - & - & - & - & - \\
\hline $06 / 15 / 2016$ & $08: 10$ & - & - & - & - & - & - & - & - & - & - & - \\
\hline
\end{tabular}


Table 8. Pesticide concentrations with measured detections in suspended sediments filtered from environmental water samples collected in the Sacramento-San Joaquin Delta, 2015-16.-Continued

[Numbers in brackets are U.S. Geological Survey (USGS) National Water Information System (NWIS) parameter codes. Concentrations are in nanogram per liter. Abbreviations: E, estimated; hh:mm, hour:minute; mm/dd/yyyy, month/day/year; —, not detected; *, storm sample]

\begin{tabular}{|c|c|c|c|c|c|c|c|c|c|c|c|c|}
\hline $\begin{array}{l}\text { Sample date } \\
\text { (mm/dd/yyyy) }\end{array}$ & $\begin{array}{l}\text { Sample time } \\
\text { (hh:mm) }\end{array}$ & $\begin{array}{c}\text { Bifenthrin } \\
\text { [65067] }\end{array}$ & $\begin{array}{c}\text { Cyhalothrin } \\
\text { (all isomers) } \\
\text { [68354] }\end{array}$ & $\begin{array}{c}\text { DCPA } \\
{[65076]}\end{array}$ & $\begin{array}{c}\text { Dithiopyr } \\
\text { [51837] }\end{array}$ & $\begin{array}{c}\text { Metalaxyl } \\
\text { [68437] }\end{array}$ & $\begin{array}{l}\text { Metolachlor } \\
\text { [65090] }\end{array}$ & $\begin{array}{c}\text { Oxyfluorfen } \\
\text { [65093] }\end{array}$ & $\begin{array}{c}\text { p,p'-DDE } \\
\text { [65095] }\end{array}$ & $\begin{array}{c}\text { Pendimethalin } \\
\text { [65098] }\end{array}$ & $\begin{array}{l}\text { Permethrin } \\
\text { [65099] }\end{array}$ & $\begin{array}{c}\text { Prodiamine } \\
\text { [51844] }\end{array}$ \\
\hline \multicolumn{13}{|c|}{ San Joaquin River at Buckley Cove } \\
\hline $07 / 28 / 2015$ & $11: 10$ & - & - & - & - & - & - & - & - & - & - & - \\
\hline $08 / 18 / 2015$ & $11: 10$ & - & - & - & - & - & - & - & - & - & 2.7 & - \\
\hline $09 / 23 / 2015$ & $10: 45$ & - & - & - & - & - & - & - & - & - & - & - \\
\hline $10 / 21 / 2015$ & $10: 50$ & - & - & - & - & - & - & - & - & - & - & - \\
\hline $11 / 10 / 2015$ & $11: 30$ & - & - & - & - & - & - & - & - & - & - & - \\
\hline $12 / 15 / 2015$ & $12: 20$ & - & - & - & - & - & - & - & - & - & - & - \\
\hline$* 01 / 19 / 2016$ & $10: 50$ & - & - & - & - & - & - & - & - & 51.1 & - & - \\
\hline $02 / 17 / 2016$ & $10: 10$ & - & - & - & - & - & - & - & - & - & - & - \\
\hline *03/07/2016 & $10: 15$ & - & - & - & - & - & - & - & - & - & - & - \\
\hline $04 / 19 / 2016$ & $10: 40$ & - & - & - & - & - & - & - & - & - & - & - \\
\hline $05 / 18 / 2016$ & $10: 45$ & - & - & - & - & - & - & - & - & - & - & - \\
\hline $06 / 15 / 2016$ & $10: 50$ & - & - & - & - & - & - & - & - & - & - & - \\
\hline \multicolumn{13}{|c|}{ San Joaquin River near Vernalis } \\
\hline $07 / 28 / 2015$ & $12: 15$ & - & - & - & - & - & - & - & - & - & - & - \\
\hline 08/18/2015 & $12: 50$ & - & - & - & - & - & - & - & - & - & - & - \\
\hline 09/23/2015 & $12: 20$ & - & - & - & - & - & - & - & - & - & - & - \\
\hline $10 / 21 / 2015$ & $12: 40$ & - & - & - & - & - & - & - & - & - & - & - \\
\hline $11 / 10 / 2015$ & $13: 00$ & - & - & - & - & - & - & - & - & - & - & - \\
\hline $12 / 15 / 2015$ & 11:00 & - & - & - & - & - & - & - & - & - & - & - \\
\hline *01/19/2016 & $12: 30$ & - & - & 86.8 & - & E1.0 & - & - & - & 27.5 & - & - \\
\hline $02 / 17 / 2016$ & $12: 00$ & - & - & - & - & - & - & - & - & - & - & - \\
\hline *03/07/2016 & $11: 50$ & - & - & - & - & - & - & - & - & - & - & - \\
\hline $04 / 19 / 2016$ & $12: 45$ & 4.3 & - & - & - & - & - & - & - & - & - & - \\
\hline $05 / 18 / 2016$ & $12: 20$ & - & - & - & - & - & - & - & - & - & - & - \\
\hline $06 / 15 / 2016$ & $12: 40$ & - & - & - & - & - & - & - & - & - & - & - \\
\hline
\end{tabular}


Table 8. Pesticide concentrations with measured detections in suspended sediments filtered from environmental water samples collected in the Sacramento-San Joaquin Delta, 2015-16.-Continued

[Numbers in brackets are U.S. Geological Survey (USGS) National Water Information System (NWIS) parameter codes. Concentrations are in nanogram per liter. Abbreviations: E, estimated; hh:mm, hour:minute; mm/dd/yyyy, month/day/year; —, not detected; *, storm sample]

\begin{tabular}{|c|c|c|c|c|c|c|c|c|c|c|c|c|}
\hline $\begin{array}{l}\text { Sample date } \\
\text { (mm/dd/yyyy) }\end{array}$ & $\begin{array}{l}\text { Sample time } \\
\text { (hh:mm) }\end{array}$ & $\begin{array}{l}\text { Bifenthrin } \\
\text { [65067] }\end{array}$ & $\begin{array}{l}\text { Cyhalothrin } \\
\text { (all isomers) } \\
\text { [68354] }\end{array}$ & $\begin{array}{c}\text { DCPA } \\
{[65076]}\end{array}$ & $\begin{array}{c}\text { Dithiopyr } \\
\text { [51837] }\end{array}$ & $\begin{array}{c}\text { Metalaxyl } \\
\text { [68437] }\end{array}$ & $\begin{array}{c}\text { Metolachlor } \\
\text { [65090] }\end{array}$ & $\begin{array}{c}\text { Oxyfluorfen } \\
\text { [65093] }\end{array}$ & $\begin{array}{c}\text { p,p'-DDE } \\
\text { [65095] }\end{array}$ & $\begin{array}{l}\text { Pendimethalin } \\
\text { [65098] }\end{array}$ & $\begin{array}{l}\text { Permethrin } \\
\text { [65099] }\end{array}$ & $\begin{array}{c}\text { Prodiamine } \\
\text { [51844] }\end{array}$ \\
\hline \multicolumn{13}{|c|}{ Ulatis Creek at Browns Road } \\
\hline $07 / 28 / 2015$ & $14: 20$ & - & - & - & - & - & - & - & - & - & - & - \\
\hline 08/18/2015 & $15: 00$ & - & - & - & - & - & - & - & - & - & 3.2 & - \\
\hline $09 / 23 / 2015$ & $14: 15$ & - & - & - & - & - & - & - & - & - & - & - \\
\hline $10 / 21 / 2015$ & $15: 00$ & - & - & - & - & - & - & - & - & - & - & - \\
\hline $11 / 10 / 2015$ & $15: 30$ & - & - & - & - & - & - & - & - & - & - & - \\
\hline $12 / 15 / 2015$ & $08: 50$ & - & - & - & - & - & - & - & - & - & - & - \\
\hline *01/19/2016 & $15: 00$ & 19 & - & - & 9.6 & - & - & 23.2 & 5.6 & 265 & - & 9.6 \\
\hline $02 / 17 / 2016$ & $14: 10$ & - & - & - & - & - & - & - & - & - & - & - \\
\hline *03/07/2016 & 08:30 & 3.5 & 15.2 & - & - & - & - & - & - & - & 26.4 & - \\
\hline $04 / 19 / 2016$ & $15: 10$ & - & - & - & - & - & - & E2.9 & - & - & - & - \\
\hline $05 / 18 / 2016$ & $14: 20$ & - & - & - & - & - & 17.8 & - & - & - & - & - \\
\hline $06 / 15 / 2016$ & $14: 40$ & - & - & - & - & - & 4.1 & - & - & - & - & - \\
\hline
\end{tabular}


Table 9. Whole water pesticide concentrations for all compounds with detections in water and suspended sediment filtered from environmental water samples collected in the Sacramento-San Joaquin Delta, 2015-16.

[Table contains concentrations for all compounds with at least one detection in the suspended sediment filtered from environmental water. The whole water concentration is the sum of the suspended-sediment concentration and the dissolved concentration. Numbers in brackets are U.S. Geological Survey (USGS) National Water Information System (NWIS) parameter codes. Concentrations are in nanogram per liter (ng/L). Whole water concentrations are listed in bold. Abbreviations: E, estimated; hh:mm, hour:minute; $\mathrm{mm} / \mathrm{dd} / \mathrm{yyyy}$, month/day/year; —, not detected; *, storm sample]

\begin{tabular}{|c|c|c|c|c|c|c|c|c|c|c|c|c|c|}
\hline \multirow{2}{*}{$\begin{array}{l}\text { Sample date } \\
\text { (mm/dd/yyyy) }\end{array}$} & \multirow{2}{*}{$\begin{array}{c}\text { Sample } \\
\text { time } \\
\text { (hh:mm) }\end{array}$} & \multicolumn{3}{|c|}{$\begin{array}{l}\text { Bifenthrin } \\
\text { [65067] }\end{array}$} & \multicolumn{3}{|c|}{$\begin{array}{c}\text { Cyhalothrin } \\
\text { (all isomers) } \\
\text { [68354] }\end{array}$} & \multicolumn{3}{|c|}{$\begin{array}{c}\text { DCPA } \\
{[65076]}\end{array}$} & \multicolumn{3}{|c|}{$\begin{array}{c}\text { Dithiopyr } \\
\text { [51837] }\end{array}$} \\
\hline & & $\begin{array}{c}\text { Suspended } \\
\text { sediment }\end{array}$ & Dissolved & $\begin{array}{l}\text { Whole } \\
\text { water }\end{array}$ & $\begin{array}{c}\text { Suspended } \\
\text { sediment }\end{array}$ & Dissolved & $\begin{array}{l}\text { Whole } \\
\text { water }\end{array}$ & $\begin{array}{c}\text { Suspended } \\
\text { sediment }\end{array}$ & Dissolved & $\begin{array}{l}\text { Whole } \\
\text { water }\end{array}$ & $\begin{array}{c}\text { Suspended } \\
\text { sediment }\end{array}$ & Dissolved & $\begin{array}{l}\text { Whole } \\
\text { water }\end{array}$ \\
\hline \multicolumn{14}{|c|}{ Mokelumne River at New Hope Road } \\
\hline $07 / 28 / 2015$ & 09:50 & - & - & - & - & - & - & - & - & - & - & - & - \\
\hline $08 / 18 / 2015$ & 09:50 & - & - & - & - & - & - & - & - & - & - & - & - \\
\hline $09 / 23 / 2015$ & 09:20 & - & - & - & - & - & - & - & - & - & - & - & - \\
\hline $10 / 21 / 2015$ & 09:10 & - & - & - & - & - & - & - & - & - & - & - & - \\
\hline $11 / 10 / 2015$ & $10: 00$ & - & - & - & - & - & - & - & - & - & - & 2.3 & 2.3 \\
\hline $12 / 15 / 2015$ & $14: 00$ & - & - & - & - & - & - & - & - & - & - & 7 & 7 \\
\hline *01/19/2016 & $09: 45$ & - & - & - & - & - & - & - & - & - & - & 14.8 & 14.8 \\
\hline $02 / 17 / 2016$ & 09:00 & - & - & - & - & - & - & - & - & - & - & - & - \\
\hline *03/07/2016 & $13: 30$ & - & - & - & - & - & - & - & - & - & - & 17.4 & 17.4 \\
\hline $04 / 19 / 2016$ & 09:20 & - & - & - & - & - & - & - & - & - & - & 2.1 & 2.1 \\
\hline $05 / 18 / 2016$ & 09:30 & - & - & - & - & - & - & - & - & - & - & - & - \\
\hline $06 / 15 / 2016$ & $09: 15$ & - & - & - & - & - & - & - & - & - & - & - & - \\
\hline \multicolumn{14}{|c|}{ Sacramento River at Hood } \\
\hline $07 / 28 / 2015$ & $08: 45$ & - & - & - & - & - & - & - & - & - & - & 7.9 & 7.9 \\
\hline $08 / 18 / 2015$ & $08: 40$ & - & 2.9 & 2.9 & - & - & - & - & - & - & - & - & - \\
\hline $09 / 23 / 2015$ & 08:30 & - & - & - & - & - & - & - & - & - & - & - & - \\
\hline $10 / 21 / 2015$ & 08:00 & - & - & - & - & - & - & - & - & - & - & - & - \\
\hline $11 / 10 / 2015$ & 09:00 & - & - & - & - & - & - & - & - & - & - & - & - \\
\hline $12 / 15 / 2015$ & $15: 00$ & - & - & - & - & - & - & - & - & - & - & 3.5 & 3.5 \\
\hline *01/19/2016 & 08:30 & - & - & - & - & - & - & - & - & - & - & - & - \\
\hline $02 / 17 / 2016$ & 08:00 & - & - & - & - & - & - & - & - & - & - & - & - \\
\hline *03/07/2016 & $14: 20$ & - & - & - & - & - & - & - & - & - & - & 12 & 12 \\
\hline $04 / 19 / 2016$ & $08: 20$ & - & - & - & - & - & - & - & - & - & - & - & - \\
\hline $05 / 18 / 2016$ & 08:30 & - & - & - & - & - & - & - & - & - & - & - & - \\
\hline $06 / 15 / 2016$ & $08: 10$ & - & - & - & - & - & - & - & - & - & - & - & - \\
\hline
\end{tabular}


Table 9. Whole water pesticide concentrations for all compounds with detections in water and suspended sediment filtered from environmental water samples collected in the Sacramento-San Joaquin Delta, 2015-16.-Continued

[Table contains concentrations for all compounds with at least one detection in the suspended sediment filtered from environmental water. The whole water concentration is the sum of the suspended-sediment concentration and the dissolved concentration. Numbers in brackets are U.S. Geological Survey (USGS) National Water Information System (NWIS) parameter codes. Concentrations are in nanogram per liter (ng/L). Whole water concentrations are listed in bold. Abbreviations: E, estimated; hh:mm, hour:minute; mm/dd/yyyy, month/day/year; —, not detected; *, storm sample]

\begin{tabular}{|c|c|c|c|c|c|c|c|c|c|c|c|c|c|}
\hline \multirow{2}{*}{$\begin{array}{l}\text { Sample date } \\
\text { (mm/dd/yyyy) }\end{array}$} & \multirow{2}{*}{$\begin{array}{l}\text { Sample } \\
\text { time } \\
\text { (hh:mm) }\end{array}$} & \multicolumn{3}{|c|}{$\begin{array}{l}\text { Bifenthrin } \\
\text { [65067] }\end{array}$} & \multicolumn{3}{|c|}{$\begin{array}{c}\text { Cyhalothrin } \\
\text { (all isomers) } \\
\text { [68354] }\end{array}$} & \multicolumn{3}{|c|}{$\begin{array}{c}\text { DCPA } \\
{[65076]}\end{array}$} & \multicolumn{3}{|c|}{$\begin{array}{c}\text { Dithiopyr } \\
\text { [51837] }\end{array}$} \\
\hline & & $\begin{array}{c}\text { Suspended } \\
\text { sediment }\end{array}$ & Dissolved & $\begin{array}{l}\text { Whole } \\
\text { water }\end{array}$ & $\begin{array}{c}\text { Suspended } \\
\text { sediment }\end{array}$ & Dissolved & $\begin{array}{l}\text { Whole } \\
\text { water }\end{array}$ & $\begin{array}{c}\text { Suspended } \\
\text { sediment }\end{array}$ & Dissolved & $\begin{array}{l}\text { Whole } \\
\text { water }\end{array}$ & $\begin{array}{c}\text { Suspended } \\
\text { sediment }\end{array}$ & Dissolved & $\begin{array}{l}\text { Whole } \\
\text { water }\end{array}$ \\
\hline \multicolumn{14}{|c|}{ San Joaquin River at Buckley Cove } \\
\hline $07 / 28 / 2015$ & $11: 10$ & - & - & - & - & - & - & - & - & - & - & 9.4 & 9.4 \\
\hline $08 / 18 / 2015$ & $11: 10$ & - & - & - & - & - & - & - & - & - & - & 2.5 & 2.5 \\
\hline $09 / 23 / 2015$ & $10: 45$ & - & - & - & - & - & - & - & - & - & - & - & - \\
\hline $10 / 21 / 2015$ & $10: 50$ & - & - & - & - & - & - & - & - & - & - & - & - \\
\hline $11 / 10 / 2015$ & $11: 30$ & - & - & - & - & - & - & - & - & - & - & - & - \\
\hline $12 / 15 / 2015$ & $12: 20$ & - & - & - & - & - & - & - & - & - & - & 2.9 & 2.9 \\
\hline *01/19/2016 & $10: 50$ & - & - & - & - & - & - & - & - & - & - & 14.1 & 14.1 \\
\hline $02 / 17 / 2016$ & $10: 10$ & - & - & - & - & - & - & - & - & - & - & 22.1 & 22.1 \\
\hline *03/07/2016 & $10: 15$ & - & - & - & - & - & - & - & - & - & - & 57.5 & $\mathbf{5 7 . 5}$ \\
\hline $04 / 19 / 2016$ & $10: 40$ & - & - & - & - & - & - & - & - & - & - & 19.9 & 19.9 \\
\hline 05/18/2016 & $10: 45$ & - & - & - & - & - & - & - & - & - & - & 4 & 4 \\
\hline $06 / 15 / 2016$ & $10: 50$ & - & - & - & - & - & - & - & - & - & - & - & - \\
\hline \multicolumn{14}{|c|}{ San Joaquin River near Vernalis } \\
\hline $07 / 28 / 2015$ & $12: 15$ & - & - & - & - & - & - & - & - & - & - & 7.2 & 7.2 \\
\hline $08 / 18 / 2015$ & $12: 50$ & - & - & - & - & - & - & - & - & - & - & 1.9 & 1.9 \\
\hline $09 / 23 / 2015$ & $12: 20$ & - & - & - & - & - & - & - & - & - & - & - & - \\
\hline $10 / 21 / 2015$ & $12: 40$ & - & - & - & - & - & - & - & - & - & - & - & - \\
\hline $11 / 10 / 2015$ & $13: 00$ & - & - & - & - & - & - & - & - & - & - & 3.1 & 3.1 \\
\hline $12 / 15 / 2015$ & $11: 00$ & - & - & - & - & - & - & - & - & - & - & 14.1 & 14.1 \\
\hline *01/19/2016 & $12: 30$ & - & - & - & - & - & - & 86.8 & - & 86.8 & - & 50.4 & 50.4 \\
\hline 02/17/2016 & $12: 00$ & - & - & - & - & - & - & - & - & - & - & 15.2 & 15.2 \\
\hline *03/07/2016 & $11: 50$ & - & - & - & - & - & - & - & - & - & - & 51 & 51 \\
\hline 04/19/2016 & $12: 45$ & 4.3 & - & 4.3 & - & - & - & - & - & - & - & 6.3 & 6.3 \\
\hline 05/18/2016 & $12: 20$ & - & - & - & - & - & - & - & - & - & - & 3.5 & 3.5 \\
\hline $06 / 15 / 2016$ & $12: 40$ & - & - & - & - & - & - & - & - & - & - & - & - \\
\hline
\end{tabular}


Table 9. Whole water pesticide concentrations for all compounds with detections in water and suspended sediment filtered from environmental water samples collected in the Sacramento-San Joaquin Delta, 2015-16.-Continued

[Table contains concentrations for all compounds with at least one detection in the suspended sediment filtered from environmental water. The whole water concentration is the sum of the suspended-sediment concentration and the dissolved concentration. Numbers in brackets are U.S. Geological Survey (USGS) National Water Information System (NWIS) parameter codes. Concentrations are in nanogram per liter (ng/L). Whole water concentrations are listed in bold. Abbreviations: E, estimated; hh:mm, hour:minute; mm/dd/yyyy, month/day/year; 一, not detected; *, storm sample]

\begin{tabular}{|c|c|c|c|c|c|c|c|c|c|c|c|c|c|}
\hline \multirow{2}{*}{$\begin{array}{l}\text { Sample date } \\
\text { (mm/dd/yyyy) }\end{array}$} & \multirow{2}{*}{$\begin{array}{l}\text { Sample } \\
\text { time } \\
\text { (hh:mm) }\end{array}$} & \multicolumn{3}{|c|}{$\begin{array}{l}\text { Bifenthrin } \\
\text { [65067] }\end{array}$} & \multicolumn{3}{|c|}{$\begin{array}{c}\text { Cyhalothrin } \\
\text { (all isomers) } \\
\text { [68354] }\end{array}$} & \multicolumn{3}{|c|}{$\begin{array}{c}\text { DCPA } \\
{[65076]}\end{array}$} & \multicolumn{3}{|c|}{$\begin{array}{c}\text { Dithiopyr } \\
\text { [51837] }\end{array}$} \\
\hline & & $\begin{array}{l}\text { Suspended } \\
\text { sediment }\end{array}$ & Dissolved & $\begin{array}{l}\text { Whole } \\
\text { water }\end{array}$ & $\begin{array}{c}\text { Suspended } \\
\text { sediment }\end{array}$ & Dissolved & $\begin{array}{l}\text { Whole } \\
\text { water }\end{array}$ & $\begin{array}{c}\text { Suspended } \\
\text { sediment }\end{array}$ & Dissolved & $\begin{array}{l}\text { Whole } \\
\text { water }\end{array}$ & $\begin{array}{c}\text { Suspended } \\
\text { sediment }\end{array}$ & Dissolved & $\begin{array}{l}\text { Whole } \\
\text { water }\end{array}$ \\
\hline \multicolumn{14}{|c|}{ Ulatis Creek at Browns Road } \\
\hline $07 / 28 / 2015$ & $14: 20$ & - & - & - & - & - & - & - & - & - & - & 15.4 & 15.4 \\
\hline $08 / 18 / 2015$ & $15: 00$ & - & - & - & - & - & - & - & - & - & - & 5.1 & 5.1 \\
\hline $09 / 23 / 2015$ & $14: 15$ & - & - & - & - & - & - & - & - & - & - & - & - \\
\hline $10 / 21 / 2015$ & $15: 00$ & - & - & - & - & - & - & - & - & - & - & 14.2 & 14.2 \\
\hline $11 / 10 / 2015$ & $15: 30$ & - & 33.3 & 33.3 & - & - & - & - & - & - & - & 7.6 & 7.6 \\
\hline $12 / 15 / 2015$ & $08: 50$ & - & 13.2 & 13.2 & - & - & - & - & - & - & - & 203 & 203 \\
\hline *01/19/2016 & $15: 00$ & 19 & - & 19 & - & - & - & - & - & - & 9.6 & 35.3 & 44.9 \\
\hline $02 / 17 / 2016$ & $14: 10$ & - & 11.5 & 11.5 & - & - & - & - & - & - & - & 13.1 & 13.1 \\
\hline$* 03 / 07 / 2016$ & 08:30 & 3.5 & - & 3.5 & 15.2 & - & 15.2 & - & - & - & - & 184 & 184 \\
\hline 04/19/2016 & $15: 10$ & - & - & - & - & - & - & - & - & - & - & 13 & 13 \\
\hline 05/18/2016 & $14: 20$ & - & - & - & - & - & - & - & - & - & - & 6.3 & 6.3 \\
\hline $06 / 15 / 2016$ & $14: 40$ & - & - & - & - & - & - & - & - & - & - & - & - \\
\hline \multirow{2}{*}{$\begin{array}{l}\text { Sample date } \\
\text { (mm/dd/yyyy) }\end{array}$} & \multirow{2}{*}{$\begin{array}{l}\text { Sample } \\
\text { time } \\
\text { (hh:mm) }\end{array}$} & \multicolumn{3}{|c|}{$\begin{array}{c}\text { Metalaxyl } \\
\text { [68437] }\end{array}$} & \multicolumn{3}{|c|}{$\begin{array}{c}\text { Metolachlor } \\
\text { [65090] }\end{array}$} & \multicolumn{3}{|c|}{$\begin{array}{l}\text { Oxyfluorfen } \\
\text { [65093] }\end{array}$} & \multicolumn{3}{|c|}{$\begin{array}{c}\text { p,p'-DDE } \\
\text { [65095] }\end{array}$} \\
\hline & & $\begin{array}{l}\text { Suspended } \\
\text { sediment }\end{array}$ & Dissolved & $\begin{array}{l}\text { Whole } \\
\text { water }\end{array}$ & $\begin{array}{c}\text { Suspended } \\
\text { sediment }\end{array}$ & Dissolved & $\begin{array}{l}\text { Whole } \\
\text { water }\end{array}$ & $\begin{array}{l}\text { Suspended } \\
\text { sediment }\end{array}$ & Dissolved & $\begin{array}{l}\text { Whole } \\
\text { water }\end{array}$ & $\begin{array}{l}\text { Suspended } \\
\text { sediment }\end{array}$ & Dissolved & $\begin{array}{l}\text { Whole } \\
\text { water }\end{array}$ \\
\hline \multicolumn{14}{|c|}{ Mokelumne River at New Hope Road-Continued } \\
\hline $07 / 28 / 2015$ & 09:50 & - & - & - & - & 14.2 & 14.2 & - & - & - & - & - & - \\
\hline $08 / 18 / 2015$ & 09:50 & - & - & - & - & 8 & 8 & - & - & - & - & - & - \\
\hline $09 / 23 / 2015$ & 09:20 & - & - & - & - & 4.4 & 4.4 & - & - & - & - & - & - \\
\hline $10 / 21 / 2015$ & 09:10 & - & - & - & - & - & - & - & - & - & - & - & - \\
\hline $11 / 10 / 2015$ & 10:00 & - & - & - & - & - & - & - & - & - & - & - & - \\
\hline $12 / 15 / 2015$ & 14:00 & - & - & - & - & - & - & - & 7.7 & 7.7 & - & - & - \\
\hline *01/19/2016 & $09: 45$ & - & - & - & - & - & - & - & 16.7 & 16.7 & - & - & - \\
\hline $02 / 17 / 2016$ & 09:00 & - & - & - & - & - & - & - & - & - & - & - & - \\
\hline *03/07/2016 & $13: 30$ & - & - & - & - & - & - & - & 6.3 & 6.3 & - & - & - \\
\hline
\end{tabular}


Table 9. Whole water pesticide concentrations for all compounds with detections in water and suspended sediment filtered from environmental water samples collected in the

Sacramento-San Joaquin Delta, 2015-16.-Continued

[Table contains concentrations for all compounds with at least one detection in the suspended sediment filtered from environmental water. The whole water concentration is the sum of the suspended-sediment concentration and the dissolved concentration. Numbers in brackets are U.S. Geological Survey (USGS) National Water Information System (NWIS) parameter codes. Concentrations are in nanogram per liter (ng/L). Whole water concentrations are listed in bold. Abbreviations: E, estimated; hh:mm, hour:minute; $\mathrm{mm} / \mathrm{dd} / \mathrm{yyyy}$, month/day/year; —, not detected; *, storm sample]

\begin{tabular}{|c|c|c|c|c|c|c|c|c|c|c|c|c|c|}
\hline \multirow{2}{*}{$\begin{array}{l}\text { Sample date } \\
\text { (mm/dd/yyyy) }\end{array}$} & \multirow{2}{*}{$\begin{array}{c}\text { Sample } \\
\text { time } \\
\text { (hh:mm) }\end{array}$} & \multicolumn{3}{|c|}{$\begin{array}{c}\text { Metalaxyl } \\
\text { [68437] }\end{array}$} & \multicolumn{3}{|c|}{$\begin{array}{c}\text { Metolachlor } \\
{[65090]}\end{array}$} & \multicolumn{3}{|c|}{$\begin{array}{c}\text { Oxyfluorfen } \\
\text { [65093] }\end{array}$} & \multicolumn{3}{|c|}{$\begin{array}{c}\text { p,p'-DDE } \\
\text { [65095] }\end{array}$} \\
\hline & & $\begin{array}{l}\text { Suspended } \\
\text { sediment }\end{array}$ & Dissolved & $\begin{array}{l}\text { Whole } \\
\text { water }\end{array}$ & $\begin{array}{c}\text { Suspended } \\
\text { sediment }\end{array}$ & Dissolved & $\begin{array}{l}\text { Whole } \\
\text { water }\end{array}$ & $\begin{array}{c}\text { Suspended } \\
\text { sediment }\end{array}$ & Dissolved & $\begin{array}{l}\text { Whole } \\
\text { water }\end{array}$ & $\begin{array}{l}\text { Suspended } \\
\text { sediment }\end{array}$ & Dissolved & $\begin{array}{l}\text { Whole } \\
\text { water }\end{array}$ \\
\hline \multicolumn{14}{|c|}{ Mokelumne River at New Hope Road-Continued } \\
\hline $04 / 19 / 2016$ & 09:20 & - & - & - & - & - & - & - & - & - & - & - & - \\
\hline $05 / 18 / 2016$ & $09: 30$ & - & - & - & - & - & - & - & - & - & - & - & - \\
\hline $06 / 15 / 2016$ & $09: 15$ & - & - & - & - & - & - & - & - & - & - & - & - \\
\hline \multicolumn{14}{|c|}{ Sacramento River at Hood-Continued } \\
\hline $07 / 28 / 2015$ & $08: 45$ & - & - & - & - & 13.2 & 13.2 & - & - & - & - & - & - \\
\hline $08 / 18 / 2015$ & 08:40 & - & - & - & - & 3.9 & 3.9 & - & - & - & - & - & - \\
\hline $09 / 23 / 2015$ & $08: 30$ & - & - & - & - & - & - & - & - & - & - & - & - \\
\hline $10 / 21 / 2015$ & 08:00 & - & - & - & - & - & - & - & - & - & - & - & - \\
\hline $11 / 10 / 2015$ & 09:00 & - & - & - & - & - & - & - & - & - & - & - & - \\
\hline $12 / 15 / 2015$ & $15: 00$ & - & - & - & - & - & - & - & 3.8 & 3.8 & - & - & - \\
\hline *01/19/2016 & 08:30 & - & - & - & - & - & - & - & - & - & - & - & - \\
\hline $02 / 17 / 2016$ & 08:00 & - & - & - & - & - & - & - & - & - & - & - & - \\
\hline *03/07/2016 & $14: 20$ & - & - & - & - & 13.6 & 13.6 & - & - & - & - & - & - \\
\hline 04/19/2016 & 08:20 & - & - & - & - & - & - & - & - & - & - & - & - \\
\hline $05 / 18 / 2016$ & 08:30 & - & - & - & - & 5.9 & 5.9 & - & - & - & - & - & - \\
\hline $06 / 15 / 2016$ & 08:10 & - & - & - & - & 7.1 & 7.1 & - & - & - & - & - & - \\
\hline \multicolumn{14}{|c|}{ San Joaquin River at Buckley Cove-Continued } \\
\hline $07 / 28 / 2015$ & $11: 10$ & - & - & - & - & 143 & 143 & - & - & - & - & - & - \\
\hline 08/18/2015 & $11: 10$ & - & - & - & - & 94.5 & 94.5 & - & - & - & - & - & - \\
\hline $09 / 23 / 2015$ & $10: 45$ & - & - & - & - & 43.3 & 43.3 & - & - & - & - & - & - \\
\hline $10 / 21 / 2015$ & $10: 50$ & - & - & - & - & 16.5 & 16.5 & - & - & - & - & - & - \\
\hline $11 / 10 / 2015$ & $11: 30$ & - & - & - & - & 12.2 & 12.2 & - & - & - & - & - & - \\
\hline $12 / 15 / 2015$ & $12: 20$ & - & - & - & - & 7 & 7 & - & - & - & - & - & - \\
\hline *01/19/2016 & $10: 50$ & - & 36.9 & 36.9 & - & 16.2 & 16.2 & - & 68.2 & 68.2 & - & - & - \\
\hline $02 / 17 / 2016$ & $10: 10$ & - & - & - & - & 10.8 & 10.8 & - & - & - & - & - & - \\
\hline *03/07/2016 & $10: 15$ & - & - & - & - & 42.8 & 42.8 & - & 211 & 211 & - & - & - \\
\hline
\end{tabular}


Table 9. Whole water pesticide concentrations for all compounds with detections in water and suspended sediment filtered from environmental water samples collected in the Sacramento-San Joaquin Delta, 2015-16.-Continued

[Table contains concentrations for all compounds with at least one detection in the suspended sediment filtered from environmental water. The whole water concentration is the sum of the suspended-sediment concentration and the dissolved concentration. Numbers in brackets are U.S. Geological Survey (USGS) National Water Information System (NWIS) parameter codes. Concentrations are in nanogram per liter (ng/L). Whole water concentrations are listed in bold. Abbreviations: E, estimated; hh:mm, hour:minute; mm/dd/yyyy, month/day/year; —, not detected; *, storm sample]

\begin{tabular}{|c|c|c|c|c|c|c|c|c|c|c|c|c|c|}
\hline \multirow{2}{*}{$\begin{array}{l}\text { Sample date } \\
\text { (mm/dd/yyyy) }\end{array}$} & \multirow{2}{*}{$\begin{array}{c}\text { Sample } \\
\text { time } \\
\text { (hh:mm) }\end{array}$} & \multicolumn{3}{|c|}{$\begin{array}{c}\text { Metalaxyl } \\
\text { [68437] }\end{array}$} & \multicolumn{3}{|c|}{$\begin{array}{c}\text { Metolachlor } \\
\text { [65090] }\end{array}$} & \multicolumn{3}{|c|}{$\begin{array}{c}\text { Oxyfluorfen } \\
\text { [65093] }\end{array}$} & \multicolumn{3}{|c|}{$\begin{array}{c}\text { p,p'-DDE } \\
\text { [65095] }\end{array}$} \\
\hline & & $\begin{array}{c}\text { Suspended } \\
\text { sediment }\end{array}$ & Dissolved & $\begin{array}{l}\text { Whole } \\
\text { water }\end{array}$ & $\begin{array}{c}\text { Suspended } \\
\text { sediment }\end{array}$ & Dissolved & $\begin{array}{l}\text { Whole } \\
\text { water }\end{array}$ & $\begin{array}{c}\text { Suspended } \\
\text { sediment }\end{array}$ & Dissolved & $\begin{array}{l}\text { Whole } \\
\text { water }\end{array}$ & $\begin{array}{c}\text { Suspended } \\
\text { sediment }\end{array}$ & Dissolved & $\begin{array}{l}\text { Whole } \\
\text { water }\end{array}$ \\
\hline \multicolumn{14}{|c|}{ San Joaquin River at Buckley Cove-Continued } \\
\hline $04 / 19 / 2016$ & $10: 40$ & - & - & - & - & 55.6 & 55.6 & - & 22.6 & 22.6 & - & - & - \\
\hline $05 / 18 / 2016$ & $10: 45$ & - & - & - & - & 42.6 & 42.6 & - & - & - & - & - & - \\
\hline $06 / 15 / 2016$ & $10: 50$ & - & - & - & - & 83.5 & 83.5 & - & - & - & - & - & - \\
\hline \multicolumn{14}{|c|}{ San Joaquin River near Vernalis-Continued } \\
\hline $07 / 28 / 2015$ & $12: 15$ & - & - & - & - & 30.8 & 30.8 & - & - & - & - & - & - \\
\hline $08 / 18 / 2015$ & $12: 50$ & - & - & - & - & 5.9 & 5.9 & - & - & - & - & - & - \\
\hline $09 / 23 / 2015$ & $12: 20$ & - & - & - & - & - & - & - & - & - & - & - & - \\
\hline $10 / 21 / 2015$ & $12: 40$ & - & - & - & - & 3.5 & 3.5 & - & - & - & - & - & - \\
\hline $11 / 10 / 2015$ & 13:00 & - & - & - & - & 6.4 & 6.4 & - & - & - & - & - & - \\
\hline $12 / 15 / 2015$ & $11: 00$ & - & - & - & - & - & - & - & - & - & - & - & - \\
\hline *01/19/2016 & $12: 30$ & E1.0 & - & E1.0 & - & 28.3 & 28.3 & - & - & - & - & - & - \\
\hline $02 / 17 / 2016$ & $12: 00$ & - & - & - & - & - & - & - & - & - & - & - & - \\
\hline *03/07/2016 & $11: 50$ & - & - & - & - & 12.6 & 12.6 & - & - & - & - & - & - \\
\hline 04/19/2016 & $12: 45$ & - & - & - & - & 9 & 9 & - & - & - & - & - & - \\
\hline $05 / 18 / 2016$ & $12: 20$ & - & - & - & - & 11.8 & 11.8 & - & - & - & - & - & - \\
\hline $06 / 15 / 2016$ & $12: 40$ & - & - & - & - & 14.8 & 14.8 & - & - & - & - & - & - \\
\hline \multicolumn{14}{|c|}{ Ulatis Creek at Browns Road-Continued } \\
\hline $07 / 28 / 2015$ & $14: 20$ & - & - & - & - & 140 & 140 & - & - & - & - & - & - \\
\hline $08 / 18 / 2015$ & $15: 00$ & - & - & - & - & 63 & 63 & - & - & - & - & - & - \\
\hline $09 / 23 / 2015$ & $14: 15$ & - & 17 & 17 & - & - & - & - & - & - & - & - & - \\
\hline $10 / 21 / 2015$ & $15: 00$ & - & - & - & - & 14.5 & 14.5 & - & - & - & - & - & - \\
\hline $11 / 10 / 2015$ & $15: 30$ & - & - & - & - & 20.9 & 20.9 & - & - & - & - & - & - \\
\hline $12 / 15 / 2015$ & 08:50 & - & - & - & - & - & - & - & 29.7 & 29.7 & - & - & - \\
\hline *01/19/2016 & $15: 00$ & - & - & - & - & 114 & 114 & 23.2 & - & 23.2 & 5.6 & - & 5.6 \\
\hline 02/17/2016 & $14: 10$ & - & - & - & - & - & - & - & 37.9 & 37.9 & - & - & - \\
\hline$* 03 / 07 / 2016$ & $08: 30$ & - & - & - & - & 57.2 & 57.2 & - & 25.7 & 25.7 & - & - & - \\
\hline
\end{tabular}


Table 9. Whole water pesticide concentrations for all compounds with detections in water and suspended sediment filtered from environmental water samples collected in the

Sacramento-San Joaquin Delta, 2015-16.-Continued

[Table contains concentrations for all compounds with at least one detection in the suspended sediment filtered from environmental water. The whole water concentration is the sum of the suspended-sediment concentration and the dissolved concentration. Numbers in brackets are U.S. Geological Survey (USGS) National Water Information System (NWIS) parameter codes. Concentrations are in nanogram per liter (ng/L). Whole water concentrations are listed in bold. Abbreviations: E, estimated; hh:mm, hour:minute; mm/dd/yyyy, month/day/year; —, not detected; *, storm sample]

\begin{tabular}{|c|c|c|c|c|c|c|c|c|c|c|c|c|c|}
\hline \multirow{2}{*}{$\begin{array}{l}\text { Sample date } \\
\text { (mm/dd/yyyy) }\end{array}$} & \multirow{2}{*}{$\begin{array}{c}\text { Sample } \\
\text { time } \\
\text { (hh:mm) }\end{array}$} & \multicolumn{3}{|c|}{$\begin{array}{c}\text { Metalaxyl } \\
\text { [68437] }\end{array}$} & \multicolumn{3}{|c|}{$\begin{array}{c}\text { Metolachlor } \\
\text { [65090] }\end{array}$} & \multicolumn{3}{|c|}{$\begin{array}{c}\text { Oxyfluorfen } \\
\text { [65093] }\end{array}$} & \multicolumn{3}{|c|}{$\begin{array}{c}\text { p,p'-DDE } \\
\text { [65095] }\end{array}$} \\
\hline & & $\begin{array}{l}\text { Suspended } \\
\text { sediment }\end{array}$ & Dissolved & $\begin{array}{l}\text { Whole } \\
\text { water }\end{array}$ & $\begin{array}{c}\text { Suspended } \\
\text { sediment }\end{array}$ & Dissolved & $\begin{array}{l}\text { Whole } \\
\text { water }\end{array}$ & $\begin{array}{c}\text { Suspended } \\
\text { sediment }\end{array}$ & Dissolved & $\begin{array}{l}\text { Whole } \\
\text { water }\end{array}$ & $\begin{array}{c}\text { Suspended } \\
\text { sediment }\end{array}$ & Dissolved & $\begin{array}{l}\text { Whole } \\
\text { water }\end{array}$ \\
\hline \multicolumn{14}{|c|}{ Ulatis Creek at Browns Road-Continued } \\
\hline $04 / 19 / 2016$ & $15: 10$ & - & - & - & - & 9.1 & 9.1 & E2.9 & 6.3 & E9.2 & - & - & - \\
\hline $05 / 18 / 2016$ & $14: 20$ & - & - & - & 17.8 & 2,630 & 2,650 & - & - & - & - & - & - \\
\hline $06 / 15 / 2016$ & $14: 40$ & - & - & - & 4.1 & 178 & 182 & - & 25.8 & 25.8 & - & - & - \\
\hline \multirow{2}{*}{$\begin{array}{l}\text { Sample date } \\
\text { (mm/dd/yyyy) }\end{array}$} & \multirow{2}{*}{$\begin{array}{l}\text { Sample } \\
\text { time } \\
\text { (hh:mm) }\end{array}$} & \multicolumn{3}{|c|}{ Pendimethalin [65098] } & \multicolumn{3}{|c|}{ Prodiamine [51844] } & \multicolumn{3}{|c|}{$\begin{array}{c}\text { Permethrin } \\
\text { [65099] }\end{array}$} & & & \\
\hline & & $\begin{array}{c}\text { Suspended } \\
\text { sediment }\end{array}$ & Dissolved & $\begin{array}{l}\text { Whole } \\
\text { water }\end{array}$ & $\begin{array}{c}\text { Suspended } \\
\text { sediment }\end{array}$ & Dissolved & $\begin{array}{l}\text { Whole } \\
\text { water }\end{array}$ & $\begin{array}{c}\text { Suspended } \\
\text { sediment }\end{array}$ & Dissolved & $\begin{array}{l}\text { Whole } \\
\text { water }\end{array}$ & & & \\
\hline \multicolumn{11}{|c|}{ Mokelumne River at New Hope Road-Continued } & & & \\
\hline $07 / 28 / 2015$ & 09:50 & - & - & - & - & - & - & - & - & - & & & \\
\hline $08 / 18 / 2015$ & 09:50 & - & - & - & - & - & - & - & - & - & & & \\
\hline $09 / 23 / 2015$ & 09:20 & - & - & - & - & - & - & - & - & - & & & \\
\hline $10 / 21 / 2015$ & 09:10 & - & - & - & - & - & - & - & - & - & & & \\
\hline $11 / 10 / 2015$ & $10: 00$ & - & - & - & - & - & - & - & - & - & & & \\
\hline $12 / 15 / 2015$ & $14: 00$ & - & - & - & - & - & - & - & - & - & & & \\
\hline$* 01 / 19 / 2016$ & $09: 45$ & - & 17.7 & 17.7 & - & - & - & - & - & - & & & \\
\hline $02 / 17 / 2016$ & 09:00 & - & - & - & - & - & - & - & - & - & & & \\
\hline$* 03 / 07 / 2016$ & $13: 30$ & - & - & - & - & - & - & - & - & - & & & \\
\hline $04 / 19 / 2016$ & 09:20 & - & - & - & - & - & - & - & - & - & & & \\
\hline $05 / 18 / 2016$ & $09: 30$ & - & - & - & - & - & - & - & - & - & & & \\
\hline $06 / 15 / 2016$ & $09: 15$ & - & - & - & - & - & - & - & - & - & & & \\
\hline \multicolumn{11}{|c|}{ Sacramento River at Hood-Continued } & & & \\
\hline $07 / 28 / 2015$ & $08: 45$ & - & - & - & - & - & - & - & - & - & & & \\
\hline $08 / 18 / 2015$ & $08: 40$ & - & - & - & - & - & - & - & - & - & & & \\
\hline $09 / 23 / 2015$ & $08: 30$ & - & - & - & - & - & - & - & - & - & & & \\
\hline $10 / 21 / 2015$ & 08:00 & - & - & - & - & - & - & - & - & - & & & \\
\hline $11 / 10 / 2015$ & 09:00 & - & - & - & - & - & - & - & - & - & & & \\
\hline
\end{tabular}


Table 9. Whole water pesticide concentrations for all compounds with detections in water and suspended sediment filtered from environmental water samples collected in the Sacramento-San Joaquin Delta, 2015-16.-Continued

[Table contains concentrations for all compounds with at least one detection in the suspended sediment filtered from environmental water. The whole water concentration is the sum of the suspended-sediment concentration and the dissolved concentration. Numbers in brackets are U.S. Geological Survey (USGS) National Water Information System (NWIS) parameter codes. Concentrations are in nanogram per liter (ng/L). Whole water concentrations are listed in bold. Abbreviations: E, estimated; hh:mm, hour:minute; mm/dd/yyyy, month/day/year; 一, not detected; *, storm sample]

\begin{tabular}{|c|c|c|c|c|c|c|c|c|c|c|}
\hline \multirow{2}{*}{$\begin{array}{l}\text { Sample date } \\
\text { (mm/dd/yyyy) }\end{array}$} & \multirow{2}{*}{$\begin{array}{l}\text { Sample } \\
\text { time } \\
\text { (hh:mm) }\end{array}$} & \multicolumn{3}{|c|}{ Pendimethalin [65098] } & \multicolumn{3}{|c|}{ Prodiamine [51844] } & \multicolumn{3}{|c|}{$\begin{array}{c}\text { Permethrin } \\
\text { [65099] }\end{array}$} \\
\hline & & $\begin{array}{l}\text { Suspended } \\
\text { sediment }\end{array}$ & Dissolved & $\begin{array}{l}\text { Whole } \\
\text { water }\end{array}$ & $\begin{array}{c}\text { Suspended } \\
\text { sediment }\end{array}$ & Dissolved & $\begin{array}{l}\text { Whole } \\
\text { water }\end{array}$ & $\begin{array}{c}\text { Suspended } \\
\text { sediment }\end{array}$ & Dissolved & $\begin{array}{l}\text { Whole } \\
\text { water }\end{array}$ \\
\hline \multicolumn{11}{|c|}{ Sacramento River at Hood-Continued } \\
\hline $12 / 15 / 2015$ & $15: 00$ & - & - & - & - & - & - & - & - & - \\
\hline$* 01 / 19 / 2016$ & 08:30 & - & - & - & - & - & - & - & - & - \\
\hline $02 / 17 / 2016$ & 08:00 & - & - & - & - & - & - & - & - & - \\
\hline *03/07/2016 & $14: 20$ & - & - & - & - & - & - & 19.2 & - & 19.2 \\
\hline $04 / 19 / 2016$ & $08: 20$ & - & - & - & - & - & - & - & - & - \\
\hline $05 / 18 / 2016$ & $08: 30$ & - & - & - & - & - & - & - & - & - \\
\hline $06 / 15 / 2016$ & $08: 10$ & - & - & - & - & - & - & - & - & - \\
\hline \multicolumn{11}{|c|}{ San Joaquin River at Buckley Cove-Continued } \\
\hline $07 / 28 / 2015$ & $11: 10$ & - & - & - & - & - & - & - & - & - \\
\hline $08 / 18 / 2015$ & $11: 10$ & - & - & - & - & - & - & 2.7 & - & 2.7 \\
\hline $09 / 23 / 2015$ & $10: 45$ & - & - & - & - & - & - & - & - & - \\
\hline $10 / 21 / 2015$ & $10: 50$ & - & - & - & - & - & - & - & - & - \\
\hline $11 / 10 / 2015$ & $11: 30$ & - & - & - & - & - & - & - & - & - \\
\hline $12 / 15 / 2015$ & $12: 20$ & - & - & - & - & - & - & - & - & - \\
\hline *01/19/2016 & $10: 50$ & 51.1 & 53.8 & 105 & - & - & - & - & - & - \\
\hline 02/17/2016 & $10: 10$ & - & - & - & - & - & - & - & - & - \\
\hline *03/07/2016 & $10: 15$ & - & 33.4 & 33.4 & - & - & - & - & - & - \\
\hline $04 / 19 / 2016$ & $10: 40$ & - & - & - & - & - & - & - & - & - \\
\hline $05 / 18 / 2016$ & $10: 45$ & - & - & - & - & - & - & - & - & - \\
\hline $06 / 15 / 2016$ & $10: 50$ & - & 14.4 & 14.4 & - & - & - & - & - & - \\
\hline \multicolumn{11}{|c|}{ San Joaquin River near Vernalis-Continued } \\
\hline $07 / 28 / 2015$ & $12: 15$ & - & - & - & - & - & - & - & - & - \\
\hline $08 / 18 / 2015$ & $12: 50$ & - & - & - & - & - & - & - & - & - \\
\hline $09 / 23 / 2015$ & $12: 20$ & - & - & - & - & - & - & - & - & - \\
\hline $10 / 21 / 2015$ & $12: 40$ & - & - & - & - & - & - & - & - & - \\
\hline $11 / 10 / 2015$ & $13: 00$ & - & - & - & - & - & - & - & - & - \\
\hline
\end{tabular}


Table 9. Whole water pesticide concentrations for all compounds with detections in water and suspended sediment filtered from environmental water samples collected in the Sacramento-San Joaquin Delta, 2015-16.-Continued

[Table contains concentrations for all compounds with at least one detection in the suspended sediment filtered from environmental water. The whole water concentration is the sum of the suspended-sediment concentration and the dissolved concentration. Numbers in brackets are U.S. Geological Survey (USGS) National Water Information System (NWIS) parameter codes. Concentrations are in nanogram per liter (ng/L). Whole water concentrations are listed in bold. Abbreviations: E, estimated; hh:mm, hour:minute; mm/dd/yyyy, month/day/year; —, not detected; *, storm sample]

\begin{tabular}{|c|c|c|c|c|c|c|c|c|c|c|}
\hline \multirow{2}{*}{$\begin{array}{l}\text { Sample date } \\
\text { (mm/dd/yyyy) }\end{array}$} & \multirow{2}{*}{$\begin{array}{c}\text { Sample } \\
\text { time } \\
\text { (hh:mm) }\end{array}$} & \multicolumn{3}{|c|}{ Pendimethalin [65098] } & \multicolumn{3}{|c|}{ Prodiamine [51844] } & \multicolumn{3}{|c|}{$\begin{array}{c}\text { Permethrin } \\
\text { [65099] }\end{array}$} \\
\hline & & $\begin{array}{l}\text { Suspended } \\
\text { sediment }\end{array}$ & Dissolved & $\begin{array}{l}\text { Whole } \\
\text { water }\end{array}$ & $\begin{array}{l}\text { Suspended } \\
\text { sediment }\end{array}$ & Dissolved & $\begin{array}{l}\text { Whole } \\
\text { water }\end{array}$ & $\begin{array}{c}\text { Suspended } \\
\text { sediment }\end{array}$ & Dissolved & $\begin{array}{l}\text { Whole } \\
\text { water }\end{array}$ \\
\hline \multicolumn{11}{|c|}{ San Joaquin River near Vernalis-Continued } \\
\hline $12 / 15 / 2015$ & $11: 00$ & - & - & - & - & - & - & - & - & - \\
\hline *01/19/2016 & $12: 30$ & 27.5 & 46 & 73.5 & - & - & - & - & - & - \\
\hline $02 / 17 / 2016$ & $12: 00$ & - & - & - & - & - & - & - & - & - \\
\hline$* 03 / 07 / 2016$ & $11: 50$ & - & 28.9 & 28.9 & - & - & - & - & - & - \\
\hline $04 / 19 / 2016$ & $12: 45$ & - & - & - & - & - & - & - & - & - \\
\hline $05 / 18 / 2016$ & $12: 20$ & - & - & - & - & - & - & - & - & - \\
\hline 06/15/2016 & $12: 40$ & - & - & - & - & - & - & - & - & - \\
\hline \multicolumn{11}{|c|}{ Ulatis Creek at Browns Road-Continued } \\
\hline $07 / 28 / 2015$ & $14: 20$ & - & - & - & - & - & - & - & - & - \\
\hline $08 / 18 / 2015$ & $15: 00$ & - & - & - & - & - & - & 3.2 & - & 3.2 \\
\hline $09 / 23 / 2015$ & $14: 15$ & - & - & - & - & - & - & - & - & - \\
\hline $10 / 21 / 2015$ & $15: 00$ & - & - & - & - & - & - & - & - & - \\
\hline $11 / 10 / 2015$ & $15: 30$ & - & - & - & - & - & - & - & - & - \\
\hline $12 / 15 / 2015$ & 08:50 & - & - & - & - & - & - & - & - & - \\
\hline *01/19/2016 & $15: 00$ & 265 & 99.6 & 365 & 9.6 & 8.5 & 18.1 & - & - & - \\
\hline $02 / 17 / 2016$ & $14: 10$ & - & - & - & - & - & - & - & - & - \\
\hline *03/07/2016 & 08:30 & - & 205 & 205 & - & E3.9 & E3.9 & 26.4 & - & 26.4 \\
\hline $04 / 19 / 2016$ & $15: 10$ & - & 33.6 & 33.6 & - & - & - & - & - & - \\
\hline $05 / 18 / 2016$ & $14: 20$ & - & - & - & - & - & - & - & - & - \\
\hline $06 / 15 / 2016$ & $14: 40$ & - & - & - & - & - & - & - & - & - \\
\hline
\end{tabular}


Table 10. Concentrations of dissolved and suspended constituents measured in environmental water samples collected in the Sacramento-San Joaquin Delta, 2015-16.

[Numbers in brackets are U.S. Geological Survey (USGS) National Water Information System (NWIS) parameter codes. Abbreviations: del., result deleted by laboratory; hh:mm, hour:minute; mg/L, milligram per liter; mm/dd/yyyy, month/day/year; <, less than ; $\mu \mathrm{g} / \mathrm{L}$, microgram per liter; - , not detected]

\begin{tabular}{|c|c|c|c|c|c|c|c|}
\hline $\begin{array}{l}\text { Sample date } \\
\text { (mm/dd/yyyy) }\end{array}$ & $\begin{array}{c}\text { Sample } \\
\text { time } \\
\text { (hh:mm) }\end{array}$ & $\begin{array}{c}\text { Dissolved } \\
\text { organic carbon } \\
{[00681]} \\
(\mathrm{mg} / \mathrm{L}) \\
\end{array}$ & $\begin{array}{c}\text { Particulate } \\
\text { inorganic carbon } \\
{[00688]} \\
(\mathrm{mg} / \mathrm{L}) \\
\end{array}$ & $\begin{array}{c}\text { Particulate } \\
\text { organic carbon } \\
{[00689]} \\
(\mathrm{mg} / \mathrm{L}) \\
\end{array}$ & $\begin{array}{c}\text { Total } \\
\text { particulate carbon } \\
{[00694]} \\
(\mathrm{mg} / \mathrm{L}) \\
\end{array}$ & $\begin{array}{c}\text { Dissolved } \\
\text { copper } \\
{[01040]} \\
(\mu \mathrm{g} / \mathrm{L}) \\
\end{array}$ & $\begin{array}{c}\text { Total } \\
\text { particulate nitrogen } \\
{[49570]} \\
(\mathrm{mg} / \mathrm{L}) \\
\end{array}$ \\
\hline $07 / 28 / 2015$ & 09:50 & 3.06 & - & 1.41 & 1.41 & 1.3 & 0.19 \\
\hline $08 / 18 / 2015$ & 09:50 & 2.12 & - & $<0.86$ & 0.86 & 0.96 & 0.099 \\
\hline $10 / 21 / 2015$ & 09:10 & 2.13 & - & $<0.21$ & 0.21 & 1.1 & 0.033 \\
\hline $11 / 10 / 2015$ & $10: 00$ & 2.2 & - & $<0.20$ & 0.2 & 1.2 & 0.031 \\
\hline $12 / 15 / 2015$ & $14: 00$ & 2.65 & - & $<0.30$ & 0.3 & 0.99 & 0.049 \\
\hline $01 / 19 / 2016$ & 09:45 & 2.94 & - & $<0.62$ & 0.62 & 1.4 & 0.088 \\
\hline $02 / 17 / 2016$ & 09:00 & 2.26 & - & $<0.76$ & 0.76 & 1.5 & 0.094 \\
\hline $06 / 15 / 2016$ & $09: 15$ & 2.68 & - & $<0.51$ & 0.51 & 1.3 & 0.06 \\
\hline \multicolumn{8}{|c|}{ Sacramento River at Hood } \\
\hline $07 / 28 / 2015$ & $08: 45$ & 1.81 & - & 0.36 & 0.36 & 1.2 & 0.049 \\
\hline $08 / 18 / 2015$ & $08: 40$ & 1.81 & - & $<0.11$ & 0.11 & 1.7 & - \\
\hline 09/23/2015 & $08: 30$ & 1.59 & - & $<0.11$ & 0.11 & 1.9 & - \\
\hline $10 / 21 / 2015$ & 08:00 & 1.64 & - & $<0.17$ & 0.17 & 1.3 & - \\
\hline $11 / 10 / 2015$ & 09:00 & 1.78 & - & $<0.26$ & 0.26 & 1.3 & 0.04 \\
\hline $12 / 15 / 2015$ & $15: 00$ & 2.04 & - & $<0.51$ & 0.51 & 1.0 & 0.084 \\
\hline \multicolumn{8}{|c|}{ San Joaquin River at Buckley Cove } \\
\hline 07/28/2015 & $11: 10$ & 5.04 & - & 1.28 & 1.28 & 2.0 & 0.233 \\
\hline $08 / 18 / 2015$ & $11: 10$ & 4.82 & - & $<0.44$ & 0.44 & 1.8 & 0.081 \\
\hline $09 / 23 / 2015$ & $10: 45$ & 4.28 & - & $<0.43$ & 0.43 & 1.9 & 0.08 \\
\hline $10 / 21 / 2015$ & $10: 50$ & 3.54 & - & $<0.35$ & 0.35 & 1.5 & 0.061 \\
\hline $11 / 10 / 2015$ & $11: 30$ & 2.72 & - & $<0.19$ & 0.19 & 1.1 & - \\
\hline $12 / 15 / 2015$ & $12: 20$ & 3.35 & 0.06 & 1.12 & 1.18 & 1.5 & 0.145 \\
\hline 01/19/2016 & $10: 50$ & 7.65 & 0.03 & 2.35 & 2.38 & 3.3 & 0.319 \\
\hline $02 / 17 / 2016$ & $10: 10$ & 8.37 & - & $<0.89$ & 0.89 & 3.7 & 0.123 \\
\hline 03/07/2016 & $10: 15$ & 7.37 & - & $<0.54$ & 0.54 & 4.4 & 0.075 \\
\hline 04/19/2016 & $10: 40$ & 4.8 & - & $<1.30$ & 1.3 & 1.9 & 0.218 \\
\hline 05/18/2016 & $10: 45$ & 3.23 & - & $<1.51$ & 1.51 & 1.2 & 0.192 \\
\hline $06 / 15 / 2016$ & $10: 50$ & 3.85 & - & $<2.17$ & 2.17 & 1.6 & 0.303 \\
\hline
\end{tabular}


Table 10. Concentrations of dissolved and suspended constituents measured in environmental water samples collected in the Sacramento-San Joaquin Delta, 2015-16.-Continued

[Numbers in brackets are U.S. Geological Survey (USGS) National Water Information System (NWIS) parameter codes. Abbreviations: del., result deleted by laboratory; hh:mm, hour:minute; mg/L, milligram per liter; mm/dd/yyyy, month/day/year; <, less than ; $\mu \mathrm{g} / \mathrm{L}$, microgram per liter; —, not detected]

\begin{tabular}{|c|c|c|c|c|c|c|c|}
\hline $\begin{array}{l}\text { Sample date } \\
\text { (mm/dd/yyyy) }\end{array}$ & $\begin{array}{c}\text { Sample } \\
\text { time } \\
\text { (hh:mm) }\end{array}$ & $\begin{array}{c}\text { Dissolved } \\
\text { organic carbon } \\
{[00681]} \\
(\mathrm{mg} / \mathrm{L}) \\
\end{array}$ & $\begin{array}{c}\text { Particulate } \\
\text { inorganic carbon } \\
{[00688]} \\
(\mathrm{mg} / \mathrm{L}) \\
\end{array}$ & $\begin{array}{c}\text { Particulate } \\
\text { organic carbon } \\
{[00689]} \\
(\mathrm{mg} / \mathrm{L}) \\
\end{array}$ & $\begin{array}{c}\text { Total } \\
\text { particulate carbon } \\
{[00694]} \\
(\mathrm{mg} / \mathrm{L}) \\
\end{array}$ & $\begin{array}{c}\text { Dissolved } \\
\text { copper } \\
{[01040]} \\
(\mu \mathrm{g} / \mathrm{L}) \\
\end{array}$ & $\begin{array}{c}\text { Total } \\
\text { particulate nitrogen } \\
{[49570]} \\
(\mathrm{mg} / \mathrm{L}) \\
\end{array}$ \\
\hline \multicolumn{8}{|c|}{ San Joaquin River near Vernalis } \\
\hline $07 / 28 / 2015$ & $12: 15$ & 2.6 & - & 0.79 & 0.79 & 0.86 & 0.178 \\
\hline 08/18/2015 & $12: 50$ & 2.27 & - & $<0.15$ & 0.15 & - & - \\
\hline $09 / 23 / 2015$ & $12: 20$ & 2.55 & - & $<0.15$ & 0.15 & 0.92 & - \\
\hline $10 / 21 / 2015$ & $12: 40$ & 2.23 & - & $<0.18$ & 0.18 & 0.81 & - \\
\hline $11 / 10 / 2015$ & 13:00 & 2.38 & - & $<0.29$ & 0.29 & 0.88 & 0.042 \\
\hline $12 / 15 / 2015$ & 11:00 & 2.54 & - & $<0.29$ & 0.29 & - & 0.05 \\
\hline $01 / 19 / 2016$ & $12: 30$ & 4.47 & - & $<1.40$ & 1.4 & 1.8 & 0.193 \\
\hline 02/17/2016 & 12:00 & 4.74 & - & $<1.48$ & 1.48 & 1.2 & 0.236 \\
\hline 03/07/2016 & $11: 50$ & 5.08 & - & $<1.60$ & 1.6 & 1.6 & 0.225 \\
\hline $04 / 19 / 2016$ & $12: 45$ & 3.06 & - & $<2.99$ & 2.99 & 1.5 & 0.434 \\
\hline $05 / 18 / 2016$ & $12: 20$ & 2.89 & - & $<2.90$ & 2.9 & 0.83 & 0.461 \\
\hline $06 / 15 / 2016$ & $12: 40$ & 2.77 & - & $<4.80$ & 4.8 & 1.0 & 0.67 \\
\hline \multicolumn{8}{|c|}{ Ulatis Creek at Browns Road } \\
\hline $07 / 28 / 2015$ & $14: 20$ & 8.01 & - & 0.96 & 0.96 & 3.0 & 0.209 \\
\hline 08/18/2015 & 15:00 & 7.22 & - & $<1.66$ & 1.66 & 2.5 & 0.29 \\
\hline $09 / 23 / 2015$ & $14: 15$ & 9.97 & - & $<1.83$ & 1.83 & 2.3 & 0.346 \\
\hline $10 / 21 / 2015$ & $15: 00$ & 8.93 & - & $<1.16$ & 1.16 & 1.5 & 0.175 \\
\hline $11 / 10 / 2015$ & $15: 30$ & 4.25 & - & $<1.25$ & 1.25 & 1.2 & 0.186 \\
\hline $12 / 15 / 2015$ & 08:50 & 6.32 & 0.05 & 1.54 & 1.59 & 2.4 & 0.248 \\
\hline $01 / 19 / 2016$ & $15: 00$ & 7.77 & 0.14 & 38.5 & 38.6 & 3.8 & 4.59 \\
\hline $02 / 17 / 2016$ & 14:10 & 4.74 & - & $<1.04$ & 1.04 & 2.4 & 0.158 \\
\hline 03/07/2016 & 08:30 & 9.24 & 0.13 & 5.14 & 5.27 & 3.9 & 0.613 \\
\hline $04 / 19 / 2016$ & $15: 10$ & 6.06 & - & $<4.41$ & 4.41 & 3.6 & 0.77 \\
\hline $05 / 18 / 2016$ & $14: 20$ & 6.85 & - & $<1.69$ & 1.69 & 3.0 & 0.255 \\
\hline $06 / 15 / 2016$ & $14: 40$ & 11.6 & - & $<1.80$ & 1.8 & 4.2 & 0.323 \\
\hline
\end{tabular}


Table 11. Water quality field parameters measured in surface-water samples collected in the Sacramento-San Joaquin Delta, California, 2015-16.

[Numbers in brackets are U.S. Geological Survey (USGS) National Water Information System parameter codes. Abbreviations: hh:mm, hour:minute; $\mathrm{mg} / \mathrm{L}$, milligram per liter; mm/dd/yyyy, month/day/year; NTU, nephelometric turbidity units; ${ }^{\circ} \mathrm{C}$, degrees Celsius; $\mu \mathrm{S} / \mathrm{cm}$, microsiemens per centimeter; - , data not collected; *, storm sample]

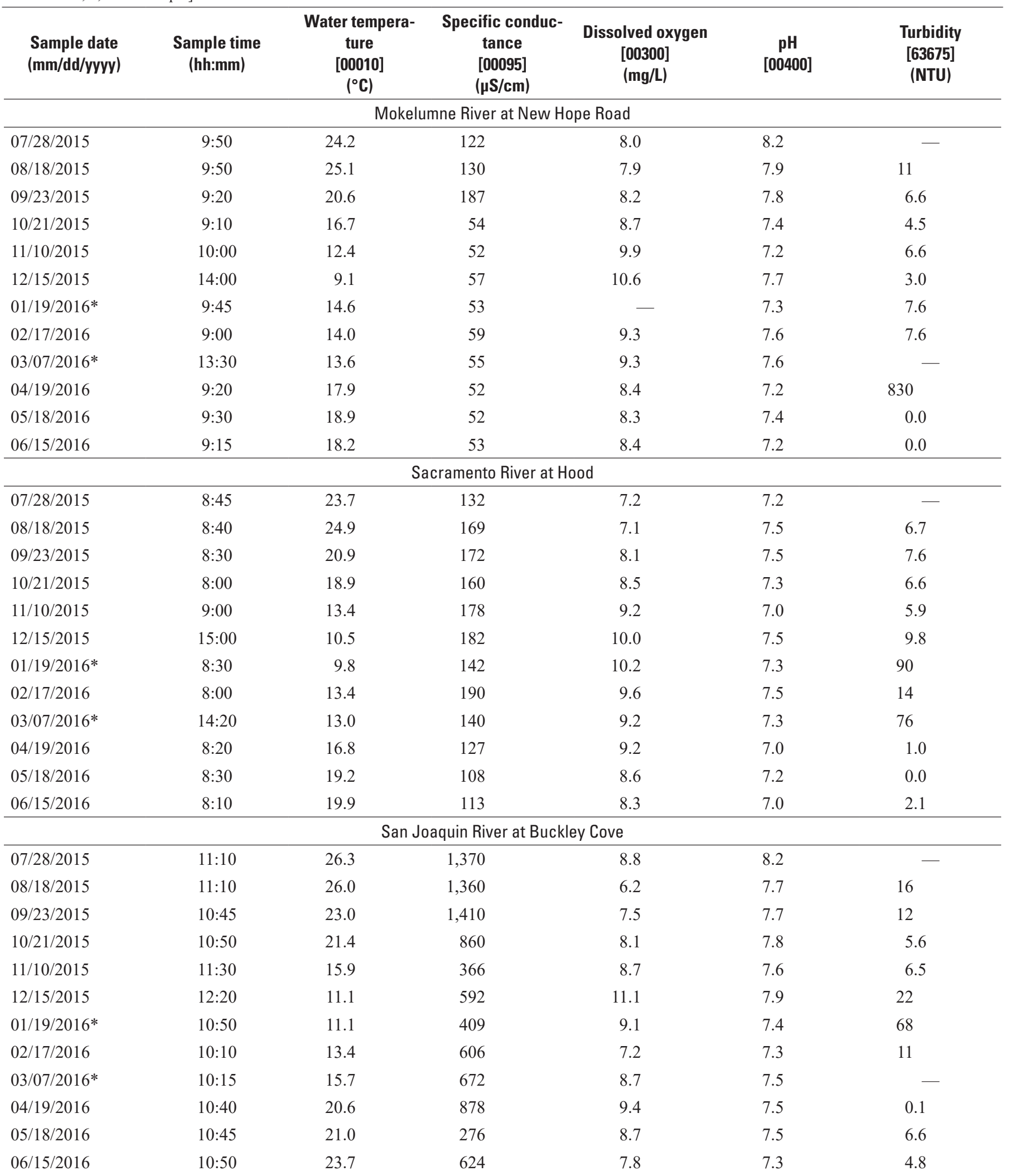


Table 11. Water quality field parameters measured in surface-water samples collected in the Sacramento-San Joaquin Delta, California, 2015-16.-Continued

[Numbers in brackets are U.S. Geological Survey (USGS) National Water Information System parameter codes. Abbreviations: hh:mm, hour:minute; $\mathrm{mg} / \mathrm{L}$, milligram per liter; mm/dd/yyyy, month/day/year; NTU, nephelometric turbidity units; ${ }^{\circ} \mathrm{C}$, degrees Celsius; $\mu \mathrm{S} / \mathrm{cm}$, microsiemens per centimeter; —, data not collected; *, storm sample]

\begin{tabular}{|c|c|c|c|c|c|c|}
\hline $\begin{array}{l}\text { Sample date } \\
\text { (mm/dd/yyyy) }\end{array}$ & $\begin{array}{c}\text { Sample time } \\
\text { (hh:mm) }\end{array}$ & $\begin{array}{c}\text { Water tempera- } \\
\text { ture } \\
{[00010]} \\
\left({ }^{\circ} \mathrm{C}\right)\end{array}$ & $\begin{array}{c}\text { Specific conduc- } \\
\text { tance } \\
{[00095]} \\
(\mu \mathrm{S} / \mathrm{cm})\end{array}$ & $\begin{array}{c}\text { Dissolved oxygen } \\
{[00300]} \\
(\mathrm{mg} / \mathrm{L})\end{array}$ & $\begin{array}{c}\mathrm{pH} \\
{[00400]}\end{array}$ & $\begin{array}{c}\text { Turbidity } \\
\text { [63675] } \\
\text { (NTU) }\end{array}$ \\
\hline \multicolumn{7}{|c|}{ San Joaquin River near Vernalis } \\
\hline $07 / 28 / 2015$ & $12: 15$ & 25.3 & 962 & 9.0 & 7.9 & - \\
\hline $08 / 18 / 2015$ & $12: 50$ & 26.6 & 668 & 8.6 & 7.8 & 6.6 \\
\hline $09 / 23 / 2015$ & $12: 20$ & 21.9 & 706 & 8.6 & 7.7 & 5.0 \\
\hline $10 / 21 / 2015$ & $12: 40$ & 18.7 & 453 & 7.5 & 7.5 & 12 \\
\hline $11 / 10 / 2015$ & $13: 00$ & 13.4 & 299 & 8.3 & 7.3 & 7.2 \\
\hline $12 / 15 / 2015$ & 11:00 & 8.8 & 476 & 10.2 & 7.8 & 6.4 \\
\hline 01/19/2016* & $12: 30$ & 12.3 & 449 & 8.4 & 7.4 & 24 \\
\hline $02 / 17 / 2016$ & $12: 00$ & 15.5 & 975 & 8.7 & 7.6 & 12 \\
\hline 03/07/2016* & $11: 50$ & 16.0 & 751 & 8.0 & 7.7 & 4.6 \\
\hline 04/19/2016 & $12: 45$ & 17.4 & 382 & 10.1 & 7.9 & 18 \\
\hline 05/18/2016 & $12: 20$ & 19.4 & 322 & 12.0 & 8.4 & 1.7 \\
\hline $06 / 15 / 2016$ & $12: 40$ & 21.2 & 433 & 14.1 & 8.6 & 7.0 \\
\hline \multicolumn{7}{|c|}{ Ulatis Creek at Browns Road } \\
\hline $07 / 28 / 2015$ & $14: 20$ & 25.2 & 695 & 7.1 & 7.9 & - \\
\hline $08 / 18 / 2015$ & $15: 00$ & 24.0 & 763 & 8.2 & 8.0 & 14 \\
\hline $09 / 23 / 2015$ & $14: 15$ & 20.9 & 1,050 & 7.2 & 7.8 & 16 \\
\hline $10 / 21 / 2015$ & $15: 00$ & 18.2 & 930 & 5.6 & 7.7 & 14 \\
\hline $11 / 10 / 2015$ & $15: 30$ & 13.8 & 1,030 & 10.2 & 8.1 & 22 \\
\hline $12 / 15 / 2015$ & $8: 50$ & 7.3 & 805 & 9.8 & 7.7 & 8.4 \\
\hline $01 / 19 / 2016^{*}$ & $15: 00$ & 12.9 & 134 & 8.7 & 7.7 & 730 \\
\hline $02 / 17 / 2016$ & $14: 10$ & 16.6 & 1,120 & 11.0 & 8.2 & 7.2 \\
\hline 03/07/2016* & $8: 30$ & 12.1 & 208 & 9.5 & 7.2 & 160 \\
\hline $04 / 19 / 2016$ & $15: 10$ & 23.3 & 819 & - & 8.8 & 2.4 \\
\hline $05 / 18 / 2016$ & $14: 20$ & 24.4 & 811 & 9.0 & 8.1 & 0.0 \\
\hline $06 / 15 / 2016$ & $14: 40$ & 19.0 & 1,040 & 9.4 & 8.2 & 11 \\
\hline
\end{tabular}




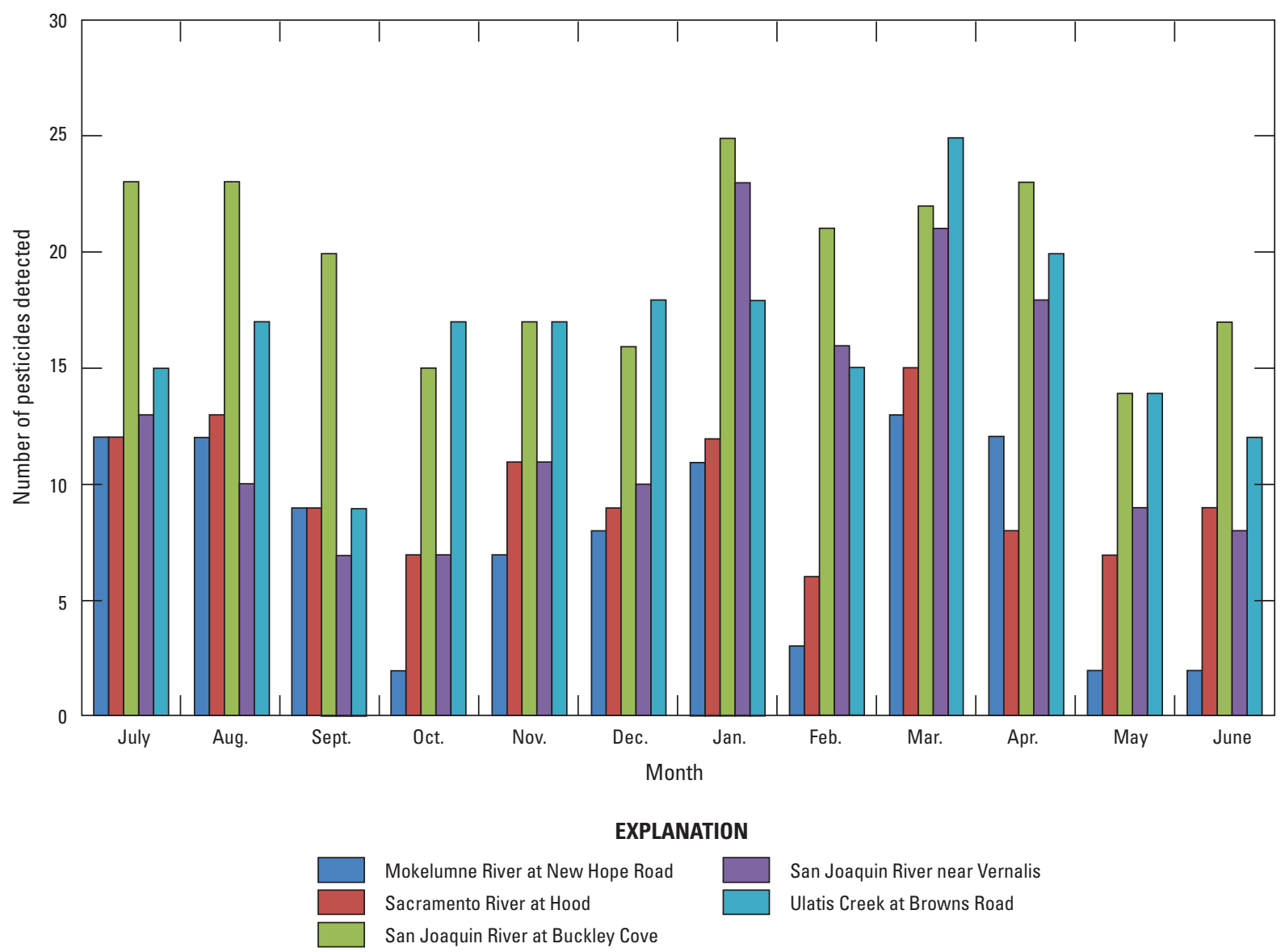

Figure 3. Numbers of pesticides detected per month in surface-water samples from July 2015 to June 2016 in the Sacramento-San Joaquin Delta, California.

\section{San Joaquin River at Buckley Cove}

A total of 37 pesticides (13 herbicides, 11 fungicides, 6 insecticides, 6 breakdown products, and 1 synergist) were detected in the water samples from this site. Nine pesticides were detected in every sample from this site (azoxystrobin, boscalid, DCPMU, diuron, fluridone, hexazinone, methoxyfenozide, metolachlor, and simazine), and nine additional pesticides (fluxapyroxad, carbendazim, chlorantraniliprole, DCPU, imidacloprid, 3,4-dichloroaniline, dithiopyr, piperonyl butoxide, and desulfinylfipronil) also were detected in at least 50 percent of the samples. The maximum pesticide concentration measured at the site was $451 \mathrm{ng} / \mathrm{L}$ for diuron in the sample collected on January 19, 2016. A minimum of 14 (May 18, 2016) and a maximum of 25 (January 19, 2016) pesticides or pesticide degradates were detected in each water sample (fig. 3). Pendimethalin was detected in the suspended sediments of the sample collected on January 19, 2016 $(51.1 \mathrm{ng} / \mathrm{L})$, and permethrin was detected in the suspended sediments collected on August 18, 2015 (2.7 ng/L).

The insecticide fipronil was detected $(13.5 \mathrm{ng} / \mathrm{L})$ in the water sample collected on July 28, 2015, at a concentration above the aquatic-life benchmark for chronic toxicity to invertebrates of $11 \mathrm{ng} / \mathrm{L}$. Imidacloprid was detected in the water samples collected on January 19, 2016 (17.8 ng/L),
April 19, 2016 (60.1 ng/L), and May 18, 2016 (13.7 ng/L), at concentrations above the aquatic-life benchmark for chronic toxicity to invertebrates of $10.0 \mathrm{ng} / \mathrm{L}$ (U.S. Environmental Protection Agency, 2017).

\section{San Joaquin River Near Vernalis}

A total of 32 pesticides (12 fungicides, 11 herbicides, 5 insecticides, and 4 breakdown products) were detected in the water samples collected from San Joaquin River near Vernalis. Three pesticides (methoxyfenozide, hexazinone, and boscalid) were detected in every sample from this site, and nine additional pesticides (azoxystrobin, metolachlor, simazine, chlorantraniliprole, dithiopyr, diuron, fluxapyroxad, DCPMU, and 3,4-dichloroaniline) were detected in at least half of the samples. The maximum pesticide concentration measured at this site was $201 \mathrm{ng} / \mathrm{L}$ for iprodione in the sample collected on February 17, 2016. A minimum of 7 (September 23, 2015, and October 21, 2015) and a maximum of 23 (January 19, 2016) pesticides or pesticide degradates were detected in each water sample (fig. 3). Imidacloprid was detected in the water samples collected on January 19, 2016 (12.4 ng/L), and April 19, $2016(11.1 \mathrm{ng} / \mathrm{L})$, at concentrations above the aquatic-life benchmark for chronic toxicity to invertebrates of $10.0 \mathrm{ng} / \mathrm{L}$ (U.S. Environmental Protection Agency, 2017). 
Four pesticides were detected in the suspended sediments of water samples collected at San Joaquin River near Vernalis. The herbicides DCPA and pendimethalin were detected in the sample collected on January 19, 2016, at concentrations of 86.8 and $27.5 \mathrm{ng} / \mathrm{L}$, respectively. The fungicide metalaxyl was detected at an estimated concentration of $1.0 \mathrm{ng} / \mathrm{L}$ in the sample collected on January 19, 2016. The insecticide bifenthrin was detected at a concentration of $4.3 \mathrm{ng} / \mathrm{L}$ in the sample collected on April 19, 2016.

\section{Ulatis Creek at Browns Road}

A total of 40 pesticides (14 herbicides, 12 fungicides, 9 insecticides, and 5 breakdown products) were detected in water samples collected from Ulatis Creek at Browns Road. Three pesticides were detected in every sample (boscalid, chlorantraniliprole, and imidacloprid), and 11 additional pesticides were detected in at least half of the samples. The maximum concentration measured at Ulatis Creek was $2,630 \mathrm{ng} / \mathrm{L}$ for the herbicide metolachlor in the sample collected on May 18, 2016. A minimum of 9 (September 23, 2015) and a maximum of 25 (March 7, 2016) pesticides or pesticide degradates were detected in each water sample (fig. 3).

Bifenthrin was detected in the samples collected on November 10, 2015 (33.3 ng/L), December 15, 2015 (13.2 ng/L), and February 17, 2016 (11.5 ng/L), at concentrations above the aquatic-life benchmark for chronic toxicity to invertebrates of $1.3 \mathrm{ng} / \mathrm{L}$. Fipronil was detected at concentrations at or above the aquatic-life benchmark for chronic toxicity to invertebrates of $11 \mathrm{ng} / \mathrm{L}$ in the samples collected on October 21, 2015 (13.4 ng/L), and January 19, 2016 (11 ng/L). Imidacloprid was detected at concentrations above the aquaticlife benchmark for chronic toxicity to invertebrates of 10.0 $\mathrm{ng} / \mathrm{L}$ in seven water samples at this site (U.S. Environmental Protection Agency, 2017).

Pesticides were detected in the suspended sediments of six samples collected at Ulatis Creek. A total of nine pesticides were detected (bifenthrin, cyhalothrin, dithiopyr, metolachlor, oxyfluorfen, $p, p$ '-DDE, pendimethalin, permethrin, and prodiamine); the most frequently detected compounds being the herbicides metolachlor and oxyfluorfen (17 percent each) and the insecticides bifenthrin and permethrin (17 percent each). The storm sample collected on January 19, 2016, contained the most pesticides (six) and generally had the highest concentrations. The highest concentrations measured in the suspended sediment were $265 \mathrm{ng} / \mathrm{L}$ for pendimethalin, $26.4 \mathrm{ng} / \mathrm{L}$ for permethrin, and $23.2 \mathrm{ng} / \mathrm{L}$ for oxyfluorfen.

\section{Summary}

This study was conducted as part of the SacramentoSan Joaquin Delta (Delta) Regional Monitoring Program, which is a cooperative effort to better track beneficial-use protections and restoration efforts through the monitoring of mercury, nutrients, pathogens, and pesticides in the Delta. The U.S. Geological Survey (USGS) was responsible for collecting, analyzing, and reporting pesticide concentration data in the Delta. Samples were collected monthly at five major inputs to the Delta from July 2015 to June 2016 and analyzed for a suite of 154 pesticides and pesticide degradates, dissolved organic carbon, particulate organic carbon, particulate inorganic carbon, particulate nitrogen, and dissolved copper by the USGS. Ten samples were collected approximately mid-month, and two samples were collected following moderate-rainfall events.

Thirty-two quality-assurance and quality-control samples (replicates, blanks, and matrix spikes) were collected to validate measurements taken on environmental water samples. Twenty-seven quality-control samples satisfied quality assurance program plan requirements for the study; one blank sample analyzed for dissolved organic carbon was measured at a concentration above the reporting level, and four pesticide matrix-spike samples each contained one compound that was measured at over 130 percent recovery.

A total of 54 pesticides were detected in water samples collected during the study period (19 fungicides, 18 herbicides, 9 insecticides, 7 breakdown products, and 1 synergist). All samples contained mixtures of pesticides ranging from 2 to 25 pesticides per sample. Overall, 14 pesticides were detected in at least half of all samples. Pesticide concentrations ranged from below method detection limits listed in table 2 to 2,630 nanograms per liter for the herbicide metolachlor.

A total of 11 pesticides were detected in suspended sediments filtered from the water samples collected during the study period ( 6 herbicides, 3 insecticides, 1 fungicide, and 1 breakdown product). Overall, the most frequently detected pesticides on suspended sediments were permethrin ( 7 percent), pendimethalin ( 5 percent), and bifenthrin (5 percent). Pesticide concentrations in the suspended sediments ranged from below the method detection limits to 265 nanograms per liter for pendimethalin.

Bifenthrin, fipronil, and imidacloprid were measured at concentrations above their respective U.S. Environmental Protection Agency (EPA) aquatic-life benchmarks for chronic toxicity to invertebrates. The EPA aquatic-life benchmarks are listed only to give context to measurements; instantaneous concentrations above chronic criteria do not constitute an assessment of water quality. 


\section{References Cited}

Aquatic Science Center, 2016, Delta regional monitoring program-Quality assurance program plan: Richmond, Calif., San Francisco Estuary Institute-Aquatic Science Center, 122 p., accessed June 1, 2017, at http://www. waterboards.ca.gov/centralvalley/water_issues/delta water_quality/delta_regional_monitoring/wq_monitoring_plans/2016_0930_drmp_qapp.pdf.

Aquatic Science Center, 2018, Delta regional monitoring program charter: San Francisco Estuary Institute-Aquatic Science Center, accessed March 30, 2017, at https://www. waterboards.ca.gov/centralvalley/water issues/delta water_quality/delta_regional_monitoring/program_docs/ drmp_charter.pdf.

ASTM International, 2010, Standard practice for performing detection and quantitation estimation and data assessment utilizing DQCALC software, based on ASTM practices D6091 and D6512 of committee D19 on water: ASTM International, D7510-10, 2 p., available at https://www. astm.org/Standards/D7510.htm.

Baxter, R., Breuer, R., Brown, L., Conrad, L., Feyrer, F., Fong, S., Gehrts, K., Grimaldo, L., Herbold, B., Hrodley, P., Mueller-Solger, A., Sommer, T., and Souza, K., 2010, 2010 Pelagic organism decline work plan and synthesis of results: Interagency Ecological Program for the San Francisco Estuary, 259 p., accessed August 17, 2017, at http://www.water. ca.gov/iep/docs/FinalPOD2010Workplan12610.pdf.

Brenton, R.W., and Arnett, T.L., 1993, Methods of analysis by the U.S. Geological Survey National Water Quality Laboratory-Determination of dissolved organic carbon by uvpromoted persulfate oxidation and infrared spectrometry: U.S. Geological Survey Open-File Report 92-480, 12 p., available at https://pubs.er.usgs.gov/publication/ofr92480.

Brown, L., and Moyle, P.B., 2005, Native fishes of the Sacramento-San Joaquin drainage, California: A history of decline, in Rinne, J.N., Hughes, R.M., and Calamusso, B., eds., Historical changes in large river fish assemblages of the Americas: American Fisheries Society Symposium, 45th, Bethesda, Md., 2005, p . 75-98.

California Department of Pesticide Regulation, 2016, Pesticide use data for 2014: Sacramento, California Department of Pesticide Regulation, accessed March 21, 2016, at http:// www.cdpr.ca.gov/docs/pur/purmain.htm.

Childress, C.J.O., Foreman, W.T., Connor, B.F., and Maloney, T.J., 1999, New reporting procedures based on longterm method detection levels and some considerations for interpretations of water-quality data provided by the U.S. Geological Survey National Water Quality Laboratory: U.S. Geological Survey Open-File Report 99-193, 19 p., available at https://water.usgs.gov/owq/OFR_99-193/.
Dileanis, P.D., Bennett, K.P., and Domagalski, J.L., 2002, Occurrence and transport of diazinon in the Sacramento River, California, and selected tributaries, during three winter storms, January-February 2000: U.S. Geological Survey Water-Resources Investigations Report 02-4101, 71 p., available at https://pubs.usgs.gov/wri/wri02-4101/.

Feyrer, F., Nobriga, M., Sommer, T., 2007, Multidecadal trends for three declining fish species-Habitat patterns and mechanisms in the San Francisco Estuary, California, USA: Canadian Journal of Fisheries and Aquatic Sciences, v., 64 p. 723-734, available at http://www.nrcresearchpress.com/ doi/abs/10.1139/f07-048\#.WnzVyG_wZaQ.

Garbarino, J.R., Kanagy, L.K., and Cree, M.E., 2006, Determination of elements in natural-water, biota, sediment, and soil samples using collision/reaction cell inductively coupled plasma-mass spectrometry: U.S. Geological Survey Techniques and Methods 5-B1, 88 p., available at https://pubs. usgs.gov/tm/2006/tm5b1/.

Hladik, M.L., and Calhoun, D.L., 2012, Analysis of the herbicide diuron, three diuron degradates, and six neonicotinoid insecticides in water-Method details and application to two Georgia streams: U.S. Geological Survey Scientific Investigations Report 2012-5206, 10 p., available at https:// pubs.usgs.gov/sir/2012/5206/pdf/sir20125206.pdf.

Hladik, M.L., and McWayne, M.M., 2012, Methods of analysis - Determination of pesticides in sediment using gas chromatography/mass spectrometry: U.S. Geological Survey Techniques and Methods 5-C3, 18 p., available at https://pubs.usgs.gov/tm/tm5c3/pdf/tm5-C3.pdf.

Hladik, M.L., Smalling, K.L., and Kuivila, K.M., 2008, A multi-residue method for the analysis of pesticides and pesticide degradates in water using Oasis HLB solid-phase extraction and gas chromatography-ion trap mass spectrometry: Bulletin of Environmental Contamination and Toxicology, v. 80, p. 139-144, available at https://www.researchgate.net/ publication/5655709_A_Multi-Residue_Method_for_the Analysis_of_Pesticides_and_Pesticide_Degradates_in Water_Using_HLB_Solid-Phase_Extraction_and_Gas_ Chromatography-Ion_Trap_Mass_Spectrometry.

Hladik, M.L., Smalling, K.L., and Kuivila, K.M., 2009, Methods of analysis - Determination of pyrethroid insecticides in water and sediment using gas chromatography/ mass spectrometry: U.S. Geological Survey Techniques and Methods 5-C2, 18 p., available at https://pubs.usgs.gov/tm/ tm5c2/tm5c2.pdf.

Kratzer, C.R., Zamora, C., and Knifong, D.L., 2002, Diazinon and chlorpyrifos loads in the San Joaquin River Basin, California, January and February 2000: U.S. Geological Survey Water Resources Investigations Report 02-4103, 38 p., available at https://pubs.usgs.gov/wri/wri02-4103/. 
Kuivila, K.M., and Hladik, M.L., 2008, Understanding the occurrence and transport of current-use pesticides in the San Francisco Estuary Watershed: San Francisco Estuary and Watershed Science, v. 6, no. 3, article 2, 19 p., available at https://www.waterboards.ca.gov/waterrights/water_issues/ programs/bay_delta/docs/cmnt081712/sldmwa/kuivilaandhladik2008.pdf.

Moyle, P.B., 2002, Inland fishes of California (2d ed.): Berkeley, Calif., University of California Press, 413 p., available at https://www.waterboards.ca.gov/water_issues/programs/ tmdl/records/state_board/1998/ref2608.pdf.

National Agricultural Statistics Service, 2016, Cropland data layer: U.S. Department of Agriculture, National Agricultural Statistics Service web page, accessed April 11, 2017, at https://nassgeodata.gmu.edu/CropScape/.

Orlando, J.L., and Kuivila, K.M., 2005, Concentrations of organic contaminants detected during managed flow conditions, San Joaquin River and Old River, California, 2001: U.S. Geological Survey Data Series 120, 13 p., available at https://pubs.usgs.gov/ds/2005/120/ds120.pdf.

Orlando, J.L., McWayne, M.M., Sanders, C., and Hladik, M.L., 2013, Dissolved pesticide concentrations in the Sacramento-San Joaquin Delta and Grizzly Bay, California, 2011-12: U.S. Geological Survey Data Series 779, 24 p., available at https://pubs.er.usgs.gov/publication/ds779.

Orlando, J.L., McWayne, M.M., Sanders, C., and Hladik, M.L., 2014, Dissolved pesticide concentrations entering the Sacramento-San Joaquin Delta from the Sacramento and San Joaquin Rivers, California, 2012-13: U.S. Geological Survey Data Series 876, 28 p., available at https://pubs. er.usgs.gov/publication/ds876.

Sommer, T., and Mejia, F., 2013, A place to call home-A synthesis of Delta smelt habitat in the upper San Francisco Estuary: San Francisco Estuary and Watershed Science, v. 11, no. 2, 25 p., available at https://escholarship.org/uc/ item/32c8t244.

Sommer, T., Armor, C., Baxter, R., Breuer, R., Brown, L., Chotkowski, M., Culberson, S., Feyer, F., Gingas, M., Herbold, B., Kimmerer, W., Mueller-Solger, A., Nobriga, M., and Souza, K., 2007, The collapse of pelagic fishes in the upper San Francisco estuary: Fisheries, v. 32, p. 270-277, available at https://www.water.ca.gov/LegacyFiles/iep/docs/ pod/sommers_fish.pdf.

Timme, P.J., 1995, National Water Quality Laboratory 1995 services catalog: U.S. Geological Survey Open-File Report 95-352, 120 p., available at https://pubs.usgs.gov/ of/1995/0352/report.pdf.

U.S. Census Bureau, 2016, Census 2010: U.S. Department of Commerce, U.S. Census Bureau, accessed March 30, 2017, at https://www.census.gov/2010census/.
U.S. Environmental Protection Agency, 1992, Definition and procedure for the determination of the method detection limit, revision 1.11: Code of Federal Regulations 40, Protection of the Environment, CFR Part 136, appendix B, p. 343-346, available at https://www.gpo.gov/fdsys/pkg/CFR2011-title40-vol23/pdf/CFR-2011-title40-vol23-part136appB.pdf.

U.S. Environmental Protection Agency, 2017, Aquatic life benchmarks and ecological risk assessments for registered pesticides: U.S. Environmental Protection Agency website, accessed February 24, 2017, at https://www.epa.gov/ pesticide-science-and-assessing-pesticide-risks/aquatic-lifebenchmarks-pesticide-registration.

U.S. Geological Survey, 2014, National Land Cover Database 2011: U.S. Geological Survey website, accessed November 16, 2014, at https://www.mrlc.gov/nlcd2011.php.

U.S. Geological Survey, 2017, USGS water data for the Nation: U.S. Geological Survey National Water Information System database, accessed April 20, 2017, at https://doi. org/10.5066/F7P55KJN.

U.S. Geological Survey, variously dated, National field manual for the collection of water-quality data (version 7): U.S. Geological Survey Techniques and Methods, book 9, chaps. A1-A10, accessed April 5, 2013, at http://water.usgs. gov/owq/FieldManual/.

Werner, I., Deanovic, L.A., Markiewicz, D., Khamphanh, M., Reece, C.K., Stillway, M., and Reece, C., 2010, Monitoring acute and chronic water column toxicity in the Northern Sacramento-San Joaquin Estuary, California, USA, using the euryhaline amphipod, Hyalella Azteca: 2006 to 2007: Environmental Toxicity and Chemistry, v. 29, no. 10, p. 2190-2199, available at https://www.ncbi.nlm.nih.gov/ pubmed/20872681.

Weston, D.P., and Lydy, M.J., 2010, Urban and agricultural sources of pyrethroid insecticides to the Sacramento-San Joaquin Delta of California: Environmental Science \& Technology, v. 44, no. 5, p. 1833-1840, available at http:// pubs.acs.org/doi/abs/10.1021/es9035573.

Zamora, C., Kratzer, C.R., Majewski, M.S., and Knifong, D.L., 2003, Diazinon and chlorpyrifos loads in precipitation and urban and agricultural storm runoff during January and February 2001 in the San Joaquin River Basin, California: U.S. Geological Survey Water-Resources Investigations Report 03-4091, 56 p., available at https://pubs.usgs.gov/ wri/wri034091/.

Zhang, X., Starner, K., and Spurlock, F., 2012, Analysis of chlorpyrifos agricultural use in regions of frequent surface water detections in California, USA: Sacramento, Calif., Department of Pesticide Regulation, 12 p., available at http://www.cdpr.ca.gov/docs/emon/pubs/ehapreps/analysis_memos/zhang_chlorpyrifos_report.pdf. 
Zimmermann, C.F., Keefe, C.W., and Bashe, J., 1997, Method 440.0 - Determination of carbon and nitrogen in sediments and particulates of estuarine/coastal waters using elemental analysis: Cincinnati, Ohio, U.S. Environmental Protection Agency, Revision 1.4, sec. 11.4.2, 10 p, available at https:// cfpub.epa.gov/si/si_public_file_download.cfm?p_download_id $=525245$. 
Publishing support provided by the U.S. Geological Survey Science Publishing Network, Sacramento Publishing Service Center

For more information concerning the research of this report, contact the Director, California Water Science Center U.S. Geological Survey 6000 J Street, Placer Hall

Sacramento, California 95819

http://ca.water.usgs.gov 


\section{$\frac{\mathbb{2}}{\mathrm{C}}$}

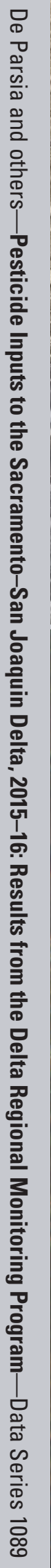

